\title{
Catalytic Asymmetric Staudinger Reactions to Form $\beta$-Lactams: An Unanticipated Dependence of Diastereoselectivity on the Choice of the Nitrogen Substituent
}

\author{
Elaine C. Lee, Brian L. Hodous, Enda Bergin, Crystal Shih, and Gregory C. Fu* \\ Department of Chemistry, Massachusetts Institute of Technology, \\ Cambridge, Massachusetts 02139
}

\section{General}

Unless otherwise noted, all materials were purchased from commercial suppliers. Toluene, $\mathrm{CH}_{2} \mathrm{Cl}_{2}$, and THF were purified by passage through a neutral alumina column under argon prior to use. Benzaldehyde, 4-fluorobenzaldehyde, 4(trifluoromethyl)benzaldehyde, $p$-anisaldehyde, $o$-tolualdehyde, and 2bromobenzaldehyde, and were distilled prior to use. Zinc metal (Strem) was activated with hydrochloric acid. $n$-Butyllithium (2.89 M in THF; Alfa Aesar) was titrated prior to use.

1,1,1-Trifluoro- $N$-sulfinylmethanesulfonamide was synthesized according to a literature procedure, ${ }^{1}$ with the modification that, after 3 days of reflux in thionyl chloride, $4 \mathrm{~N} \mathrm{HCl}$ in dioxane ( 0.5 equiv) was added, and the mixture was refluxed for an additional $12 \mathrm{~h}$. Catalyst 1 was prepared and resolved as previously reported. ${ }^{2}$ Phenyl methyl ketene, phenyl ethyl ketene, phenyl isobutyl ketene, and diphenyl ketene were synthesized according to a literature procedure, ${ }^{3}$ using $\mathrm{N}, \mathrm{N}$-dimethylethylamine as the base; in the case of phenyl methyl ketene, $\mathrm{Et}_{2} \mathrm{O}$, rather than THF, was used as the solvent.

${ }^{19} \mathrm{~F}$ NMR spectra are referenced to external trifluoroacetic anhydride at $\delta-78$.

All air- and moisture-sensitive manipulations were carried out under nitrogen using standard Schlenk techniques or in a glove box. 


\section{Synthesis of Imines}

These yields have not been optimized.

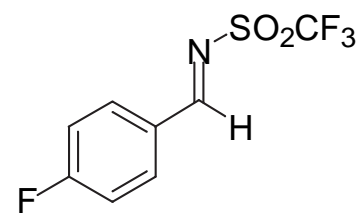

General procedure. 4-Fluorobenzaldehyde ( $211 \mathrm{mg}, 1.70 \mathrm{mmol}$; freshly distilled) and $\mathrm{CF}_{3} \mathrm{SO}_{2} \mathrm{NSO}(332 \mathrm{mg}, 2.56 \mathrm{mmol}$ ) were dissolved in anhydrous 1,2-dichloroethane $(2.7 \mathrm{~mL})$. The resulting solution was heated to reflux under nitrogen for $16 \mathrm{~h}$. The solvent was then removed under vacuum, leaving an off-white solid. The solid was dissolved in $\mathrm{CH}_{2} \mathrm{Cl}_{2}$ / pentane (1:1) and filtered through a pad of Celite. The filtrate was concentrated and re-dissolved in refluxing pentane. The desired imine crystallized upon slow cooling (white crystals; $285 \mathrm{mg}(75 \%)$ ).

${ }^{1} \mathrm{H}$ NMR $\left(500 \mathrm{MHz}, \mathrm{CDCl}_{3}\right) \delta 9.15(\mathrm{~s}, 1 \mathrm{H}), 8.13(\mathrm{~m}, 2 \mathrm{H}), 7.31(\mathrm{~m}, 2 \mathrm{H}) .{ }^{13} \mathrm{C}$ NMR $(126$ $\left.\mathrm{MHz}, \mathrm{CDCl}_{3}\right) \delta 178.4,168.6\left(\mathrm{~d},{ }^{1} \mathrm{~J}_{\mathrm{CF}}=264 \mathrm{~Hz}\right), 135.7\left(\mathrm{~d},{ }^{3} \mathrm{~J}_{\mathrm{CF}}=10.4 \mathrm{~Hz}\right), 128.2,119.3(\mathrm{q}$, $\left.{ }^{1} \mathrm{~J}_{\mathrm{CF}}=322 \mathrm{~Hz}\right), 117.6\left(\mathrm{~d},{ }^{2} \mathrm{~J}_{\mathrm{CF}}=22.4 \mathrm{~Hz}\right) .{ }^{19} \mathrm{~F}$ NMR $\left(282 \mathrm{MHz}, \mathrm{C}_{6} \mathrm{D}_{6}\right) \delta-77.9,-98.3$. HRMS (EI, m/e) calcd for $\mathrm{C}_{8} \mathrm{H}_{5} \mathrm{~F}_{4} \mathrm{NO}_{2} \mathrm{~S}(\mathrm{M}+)$ 254.9977, found 254.9981. FTIR $\left(\mathrm{CH}_{2} \mathrm{Cl}_{2}\right) 3056$, $1569,1223,740 \mathrm{~cm}^{-1}$. M.p. $61-63^{\circ} \mathrm{C}$.<smiles>O=S(=O)(/N=C/c1ccccc1)OC(F)(F)F</smiles>

The general procedure was followed: benzaldehyde $(900 \mathrm{mg}, 4.61 \mathrm{mmol})$ and $\mathrm{CF}_{3} \mathrm{SO}_{2} \mathrm{NSO}$ (489 mg, $4.61 \mathrm{mmol}$ ) in 1,2-dichloroethane $(5.0 \mathrm{~mL})$. The reaction mixture was concentrated to dryness, and the product was purified via distillation $\left(72-75^{\circ} \mathrm{C}\right.$ at $300 \mathrm{mtorr})$. Product: $785 \mathrm{mg}(77 \%)$; colorless oil that solidified upon standing.

${ }^{1} \mathrm{H}$ NMR $\left(500 \mathrm{MHz}, \mathrm{CDCl}_{3}\right) \delta 9.19(\mathrm{~s}, 1 \mathrm{H}), 8.08(\mathrm{dd}, \mathrm{J}=7.0, \mathrm{~J}=1.0 \mathrm{~Hz}, 2 \mathrm{H}), 7.80(\mathrm{~m}, 1 \mathrm{H})$, $7.62(\mathrm{t}, \mathrm{J}=7.5 \mathrm{~Hz}, 2 \mathrm{H}) .{ }^{13} \mathrm{C}$ NMR $\left(126 \mathrm{MHz}, \mathrm{CDCl}_{3}\right) \delta 180.1,137.6,132.9,131.7,129.9$, $119.3\left(\mathrm{q},{ }^{1} \mathrm{~J}_{\mathrm{CF}}=321 \mathrm{~Hz}\right) .{ }^{19} \mathrm{~F}$ NMR $\left(282 \mathrm{MHz}, \mathrm{C}_{6} \mathrm{D}_{6}\right) \delta-77.9$. HRMS (EI, m/e) calcd for $\mathrm{C}_{8} \mathrm{H}_{6} \mathrm{~F}_{3} \mathrm{NO}_{2} \mathrm{~S}(\mathrm{M}+)$ 237.0071, found 237.0055. FTIR $\left(\mathrm{CH}_{2} \mathrm{Cl}_{2}\right)$ 3058, 1595, 1564, 1364, 1223, $739 \mathrm{~cm}^{-1}$.<smiles>FC(F)(F)O/N=C/c1ccc(C(F)(F)F)cc1</smiles>

The general procedure was followed: 4-(trifluoromethyl)benzaldehyde (890 mg, 5.12 mmol; freshly distilled) and $\mathrm{CF}_{3} \mathrm{SO}_{2} \mathrm{NSO}(1.00 \mathrm{~g}, 5.12 \mathrm{mmol})$ in 1,2-dichloroethane (10 $\mathrm{mL}$ ). Product: $967 \mathrm{mg}$ (62\%); white crystalline solid.

${ }^{1} \mathrm{H}$ NMR $\left(500 \mathrm{MHz}, \mathrm{CDCl}_{3}\right) \delta 9.27(\mathrm{~s}, 1 \mathrm{H}), 8.21(\mathrm{~d}, \mathrm{~J}=8.0 \mathrm{~Hz}, 2 \mathrm{H}), 7.88(\mathrm{~d}, \mathrm{~J}=8.5 \mathrm{~Hz}$, $2 \mathrm{H}) .{ }^{13} \mathrm{C}$ NMR $\left(126 \mathrm{MHz}, \mathrm{CDCl}_{3}\right) \delta 178.6,138.1\left(\mathrm{q},{ }^{2} \mathrm{~J}_{\mathrm{CF}}=32.8 \mathrm{~Hz}\right), 134.4,132.9,126.8(\mathrm{q}$, $\left.{ }^{3} \mathrm{~J}_{\mathrm{CF}}=3.5 \mathrm{~Hz}\right), 123.2\left(\mathrm{q},{ }^{1} \mathrm{~J}_{\mathrm{CF}}=273 \mathrm{~Hz}\right), 119.3\left(\mathrm{q},{ }^{1} \mathrm{~J}_{\mathrm{CF}}=322 \mathrm{~Hz}\right) .{ }^{19} \mathrm{~F} \mathrm{NMR}\left(282 \mathrm{MHz}, \mathrm{CDCl}_{3}\right) \delta$ 
-64.2, -77.0. HRMS (EI, m/e) calcd for $\mathrm{C}_{9} \mathrm{H}_{5} \mathrm{~F}_{6} \mathrm{NO}_{2} \mathrm{~S}(\mathrm{M}+)$ 304.9945, found 304.9938 . FTIR $\left(\mathrm{CH}_{2} \mathrm{Cl}_{2}\right) 3056,1604,1266,1226,740 \mathrm{~cm}^{-1}$. M.p. $45-46^{\circ} \mathrm{C}$.

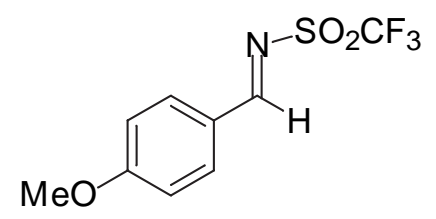

The general procedure was followed: $p$-anisaldehyde $(717 \mathrm{mg}, 5.27 \mathrm{mmol})$ and $\mathrm{CF}_{3} \mathrm{SO}_{2} \mathrm{NSO}(1.03 \mathrm{~g}, 5.27 \mathrm{mmol})$ in 1,2-dichloroethane $(10 \mathrm{~mL})$. Product: $1.00 \mathrm{~g}(71 \%)$; white crystalline solid.

${ }^{1} \mathrm{H}$ NMR $\left(500 \mathrm{MHz}, \mathrm{CDCl}_{3}\right) \delta 9.04(\mathrm{~s}, 1 \mathrm{H}), 8.04(\mathrm{~d}, \mathrm{~J}=9.0 \mathrm{~Hz}, 2 \mathrm{H}), 7.07$ (d, J=6.5 Hz, $2 \mathrm{H}), 3.96(\mathrm{~s}, 3 \mathrm{H}) .{ }^{13} \mathrm{C}$ NMR $\left(126 \mathrm{MHz}, \mathrm{CDCl}_{3}\right) \delta$ 178.6, 167.8, 135.9, 124.7, 144.7, 119.4 (q, $\left.{ }^{1} \mathrm{~J}_{\mathrm{CF}}=321 \mathrm{~Hz}\right)$, 56.2. ${ }^{19} \mathrm{~F}$ NMR $\left(282 \mathrm{MHz}, \mathrm{CDCl}_{3}\right) \delta-77.6$. HRMS (EI, m/e) calcd for $\mathrm{C}_{9} \mathrm{H}_{8} \mathrm{~F}_{3} \mathrm{NO}_{3} \mathrm{~S}(\mathrm{M}+)$ 267.0171, found 267.0167. FTIR $\left(\mathrm{CH}_{2} \mathrm{Cl}_{2}\right) 3057,1551,1220,824,740$ $\mathrm{cm}^{-1}$. M.p. $78-79{ }^{\circ} \mathrm{C}$.<smiles>Cc1ccccc1/C=N/OS(C)(F)F</smiles>

The general procedure was followed: $o$-tolualdehyde $(308 \mathrm{mg}, 2.56 \mathrm{mmol}$; freshly distilled) and $\mathrm{CF}_{3} \mathrm{SO}_{2} \mathrm{NSO}(500 \mathrm{mg}, 2.56 \mathrm{mmol})$ in 1,2-dichloroethane $(5.0 \mathrm{~mL})$. Product: $302 \mathrm{mg}(47 \%)$; off-white crystalline solid.

${ }^{1} \mathrm{H}$ NMR $\left(500 \mathrm{MHz}, \mathrm{CDCl}_{3}\right) \delta 9.46(\mathrm{~s}, 1 \mathrm{H}), 8.13(\mathrm{dd}, \mathrm{J}=7.0, \mathrm{~J}=1.5 \mathrm{~Hz}, 1 \mathrm{H}), 7.65(\mathrm{dt}$, $\mathrm{J}=6.0, \mathrm{~J}=1.5 \mathrm{~Hz}, 1 \mathrm{H}), 7.39(\mathrm{~m}, 2 \mathrm{H}), 2.70(\mathrm{~s}, 3 \mathrm{H}) .{ }^{13} \mathrm{C} \mathrm{NMR}\left(126 \mathrm{MHz}, \mathrm{CDCl}_{3}\right) \delta$ 178.6, 144.7, 137.3, 132.7, 132.4, 129.9, 127.4, $119.4\left(\mathrm{q},{ }^{1} \mathrm{~J}_{\mathrm{CF}}=321 \mathrm{~Hz}\right), 20.2 .{ }^{19} \mathrm{~F}$ NMR $(282 \mathrm{MHz}$, $\left.\mathrm{C}_{6} \mathrm{D}_{6}\right) \delta$-77.5. HRMS (EI, m/e) calcd for $\mathrm{C}_{9} \mathrm{H}_{8} \mathrm{~F}_{3} \mathrm{NO}_{2} \mathrm{~S}(\mathrm{M}+)$ 251.0228, found 251.0212. FTIR $\left(\mathrm{CH}_{2} \mathrm{Cl}_{2}\right) 3055,1558,1265,738 \mathrm{~cm}^{-1}$. M.p. $63-65^{\circ} \mathrm{C}$.<smiles>O=C(F)OS(=O)(F)(F)F</smiles>

The general procedure was followed: 2-bromobenzaldehyde (462 $\mathrm{mg}, 2.45 \mathrm{mmol}$ ) and $\mathrm{CF}_{3} \mathrm{SO}_{2} \mathrm{NSO}$ (478 mg, $2.45 \mathrm{mmol}$ ) in 1,2-dichloroethane (5.0 mL). Product: $334 \mathrm{mg}$ (44\%); off-white crystalline solid.

${ }^{1} \mathrm{H}$ NMR $\left(500 \mathrm{MHz}, \mathrm{CDCl}_{3}\right) \delta 9.63(\mathrm{~s}, 1 \mathrm{H}), 8.30(\mathrm{~d}, \mathrm{~J}=8.0 \mathrm{~Hz}, 1 \mathrm{H}), 7.77(\mathrm{~d}, \mathrm{~J}=7.5 \mathrm{~Hz}$, $1 \mathrm{H}), 7.61(\mathrm{dt}, \mathrm{J}=6.0, \mathrm{~J}=1.5 \mathrm{~Hz}, 1 \mathrm{H}), 7.52(\mathrm{t}, \mathrm{J}=8.0 \mathrm{~Hz}, 1 \mathrm{H}) .{ }^{13} \mathrm{C} \mathrm{NMR}\left(126 \mathrm{MHz} \mathrm{CDCl}_{3}\right) \delta$ 179.1 138.3, 134.6, 131.7, 131.0, 130.6, 128.7, 119.3 (q, $\left.{ }^{1} \mathrm{~J}_{\mathrm{CF}}=321 \mathrm{~Hz}\right) .{ }^{19} \mathrm{~F}$ NMR $(282 \mathrm{MHz}$, $\left.\mathrm{C}_{6} \mathrm{D}_{6}\right) \delta-77.5$. HRMS (EI, m/e) calcd for $\mathrm{C}_{8} \mathrm{H}_{5} \mathrm{BrF}_{3} \mathrm{NO}_{2} \mathrm{~S}(\mathrm{M}+)$ 314.9176, found 314.9169 . FTIR $\left(\mathrm{CH}_{2} \mathrm{Cl}_{2}\right) 3055,1582,1266,739 \mathrm{~cm}^{-1}$. M.p. $44-46{ }^{\circ} \mathrm{C}$. 


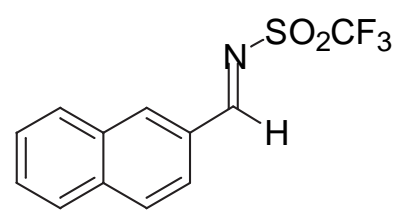

The general procedure was followed: 2-naphthaldehyde (155 $\mathrm{mg}, 0.992 \mathrm{mmol})$ and $\mathrm{CF}_{3} \mathrm{SO}_{2} \mathrm{NSO}(194 \mathrm{mg}, 0.992 \mathrm{mmol})$ in 1,2-dichloroethane $(2.0 \mathrm{~mL})$. Product: $220 \mathrm{mg}$ (77\%); pale-yellow crystalline solid.

${ }^{1} \mathrm{H}$ NMR $\left(500 \mathrm{MHz}, \mathrm{CDCl}_{3}\right) \delta 9.33(\mathrm{~s}, 1 \mathrm{H}), 8.52(\mathrm{~s}, 1 \mathrm{H}), 8.14(\mathrm{~d}, \mathrm{~J}=8.5 \mathrm{~Hz}, 1 \mathrm{H}), 8.05(\mathrm{~d}$, $\mathrm{J}=8.0 \mathrm{~Hz}, 1 \mathrm{H}), 8.00(\mathrm{~d}, \mathrm{~J}=9.0 \mathrm{~Hz}, 1 \mathrm{H}), 7.96(\mathrm{~d}, \mathrm{~J}=8.5 \mathrm{~Hz}, 1 \mathrm{H}), 7.76(\mathrm{t}, \mathrm{J}=7.0 \mathrm{~Hz}, 1 \mathrm{H}), 7.67(\mathrm{t}$, $\mathrm{J}=7.5 \mathrm{~Hz}, 1 \mathrm{H}) .{ }^{13} \mathrm{C}$ NMR $\left(126 \mathrm{MHz}, \mathrm{CDCl}_{3}\right) \delta 180.0,139.5,137.8,132.7,131.2,130.3,130.0$, 129.4, 128.5, 128.0, 124.1, $119.4\left(\mathrm{q},{ }^{1} \mathrm{~J}_{\mathrm{CF}}=321 \mathrm{~Hz}\right) .{ }^{19} \mathrm{~F}$ NMR $\left(282 \mathrm{MHz}, \mathrm{C}_{6} \mathrm{D}_{6}\right) \delta-77.8$. HRMS (EI, m/e) calcd for $\mathrm{C}_{12} \mathrm{H}_{8} \mathrm{~F}_{3} \mathrm{NO}_{2} \mathrm{~S}(\mathrm{M}+)$ 287.0228, found 287.0214. FTIR $\left(\mathrm{CH}_{2} \mathrm{Cl}_{2}\right)$ $3055,1563,1266,739 \mathrm{~cm}^{-1}$. M.p. $124-126^{\circ} \mathrm{C}$. 


\section{Catalytic Asymmetric Synthesis of trans $\beta$-Lactams}

General. The two solutions (1.0 equiv of ketene; 1.0 equiv of $N$-triflyl imine and 0.10 equiv of (-)-1) were prepared in a glove box. The rest of the procedure was conducted outside of the glove box under a nitrogen atmosphere.

Table 1, entry 1. A solution of 1,1,1-trifluoro- $N$-(phenylmethylene)methanesulfonamide $(56.4 \mathrm{mg}, 0.24 \mathrm{mmol})$ and $(-)-\mathbf{1}(8.9 \mathrm{mg}, 24 \mu \mathrm{mol})$ in toluene $(11 \mathrm{~mL})$ was cooled to $-78{ }^{\circ} \mathrm{C}$. To this solution was added a solution of phenyl ethyl ketene $(34.8 \mathrm{mg}, 0.24$ $\mathrm{mmol})$ in toluene $(1.2 \mathrm{~mL}$ ) over approximately $1 \mathrm{~min}$; the syringe was rinsed with toluene $(0.3 \mathrm{~mL})$. The resulting solution was allowed to warm to room temperature and stirred for $12 \mathrm{~h}$. The solvent was then removed by evaporation, and the residue was purified by column chromatography ( $5 \rightarrow 10 \%$ EtOAc/hexanes), which furnished the $\beta$-lactam as a colorless oil ( $54.3 \mathrm{mg}, 60 \%$; mixture of diastereomers).

The major diastereomer was isolated by column chromatography $(2.5 \%$ EtOAc/hexanes) as a colorless oil. Yield: $36.6 \mathrm{mg}(40 \%)$. HPLC analysis: $65 \%$ ee [Daicel CHIRALCEL AD-H column; $0.5 \mathrm{~mL} / \mathrm{min}$; solvent system: $1.0 \%$ isopropanol/hexanes; retention times: $10.7 \mathrm{~min}$ (major), $9.92 \mathrm{~min}$ (minor)].

Second run (same scale): Yield: $54.0 \mathrm{mg}$ (59\%). Yield of the major diastereomer: 33.0 $\operatorname{mg}(36 \%), 60 \%$ ee.

${ }^{1} \mathrm{H}$ NMR $\left(500 \mathrm{MHz}, \mathrm{C}_{6} \mathrm{D}_{6}\right) \delta 7.22(\mathrm{~m}, 2 \mathrm{H}), 6.99-7.09(\mathrm{~m}, 8 \mathrm{H}), 5.23(\mathrm{~s}, 1 \mathrm{H}), 1.51$ (qq, $\mathrm{J}=7.0, \mathrm{~J}=7.5 \mathrm{~Hz}, 1 \mathrm{H}), 0.99(\mathrm{qq}, \mathrm{J}=7.0, \mathrm{~J}=8.0 \mathrm{~Hz}, 1 \mathrm{H}), 0.45(\mathrm{t}, \mathrm{J}=7.0 \mathrm{~Hz}, 3 \mathrm{H}) .{ }^{13} \mathrm{C}$ NMR $(126$ $\left.\mathrm{MHz}, \mathrm{CDCl}_{3}\right) \delta 167.7,137.1,132.7,129.7,129.5,129.1,128.5,127.3,126.1,119.5$ (q, $\left.{ }^{1} \mathrm{~J}_{\mathrm{CF}}=323 \mathrm{~Hz}\right), 71.2,69.7,27.4,8.7 .{ }^{19} \mathrm{~F} \mathrm{NMR}\left(282 \mathrm{MHz}, \mathrm{C}_{6} \mathrm{D}_{6}\right) \delta-76.1$. FTIR (NaCl) 1818, $1413,1266,1212,740 \mathrm{~cm}^{-1}$. HRMS (ESI, m/e) calcd for $\mathrm{C}_{18} \mathrm{H}_{16} \mathrm{~F}_{3} \mathrm{NNaO}_{3} \mathrm{~S}(\mathrm{M}+\mathrm{Na})$ 406.0803, found 406.0682. $[\alpha]^{20}-104\left(c 0.26, \mathrm{CH}_{2} \mathrm{Cl}_{2}, 65 \%\right.$ ee).

Table 1, entry 2. A solution of 1,1,1-trifluoro- $N$-(phenylmethylene)methanesulfonamide (47.4 mg, $0.20 \mathrm{mmol})$ and $(-)-\mathbf{1}(7.5 \mathrm{mg}, 20 \mu \mathrm{mol})$ in $\mathrm{CH}_{2} \mathrm{Cl}_{2}(10.2 \mathrm{~mL})$ was cooled to $0{ }^{\circ} \mathrm{C}$. To this solution was added a solution of phenyl methyl ketene $(26.4 \mathrm{mg}, 0.20$ $\mathrm{mmol})$ in $\mathrm{CH}_{2} \mathrm{Cl}_{2}(1.0 \mathrm{~mL})$ over approximately $2 \mathrm{~min}$; the syringe was rinsed with $\mathrm{CH}_{2} \mathrm{Cl}_{2}(0.3 \mathrm{~mL})$. The resulting solution was allowed to warm to room temperature and stirred for $12 \mathrm{~h}$. The solvent was then removed by evaporation, and the residue was purified by column chromatography ( $5 \rightarrow 20 \%$ EtOAc/hexanes), which furnished the $\beta$-lactam as a milky oil $(60.9 \mathrm{mg}, 82 \%)$.

GC analysis: $79 \%$ ee [CP-Chirasil-Dex CB column; $170{ }^{\circ} \mathrm{C}(10 \mathrm{~min}), 0.5^{\circ} \mathrm{C} / \mathrm{min}$ to 180 ${ }^{\circ} \mathrm{C}$; 9.17 psi He; retention times: 27.0 min (major), 27.4 min (minor)].

Second run (same scale): Yield: $62.3 \mathrm{mg}(84 \%), 83 \%$ ee.

${ }^{1} \mathrm{H}$ NMR $\left(500 \mathrm{MHz}, \mathrm{C}_{6} \mathrm{D}_{6}\right)$ d 7.21-7.19 (m, 2H), 7.01-7.05 (m, 6H), $6.94(\mathrm{~m}, 2 \mathrm{H}) 5.24(\mathrm{~s}$, $1 \mathrm{H}), 0.83(\mathrm{~s}, 3 \mathrm{H}) .{ }^{13} \mathrm{C} \mathrm{NMR}\left(126 \mathrm{MHz}, \mathrm{CDCl}_{3}\right) \delta$ 168.3, 139.2, 132.8, 129.7, 129.2, 128.6, 127.0, 125.4, $119.5\left(\mathrm{q},{ }^{1} \mathrm{~J}_{\mathrm{CF}}=323 \mathrm{~Hz}\right), 71.3,65.9,20.4 .{ }^{19} \mathrm{~F} \mathrm{NMR}\left(282 \mathrm{MHz}, \mathrm{C}_{6} \mathrm{D}_{6}\right) \delta$-76.2. FTIR (NaCl) 1821, 1413, 1213, $1146 \mathrm{~cm}^{-1}$. HRMS (ESI, m/e) calcd for $\mathrm{C}_{17} \mathrm{H}_{14} \mathrm{~F}_{3} \mathrm{NNaO}_{3} \mathrm{~S}$ $(\mathrm{M}+\mathrm{Na}) 392.0803$, found 392.0525. $[\alpha]^{20}{ }_{\mathrm{D}}-100\left(\mathrm{c} 0.30, \mathrm{CH}_{2} \mathrm{Cl}_{2}, 79 \%\right.$ ee).

Table 1, entry 3. To a solution of 1,1,1-trifluoro- $N$-(phenylmethylene)methanesulfonamide $(68.1 \mathrm{mg}, 0.29 \mathrm{mmol})$ and $(-)-\mathbf{1}(10.8 \mathrm{mg}, 28.7 \mu \mathrm{mol})$ in $\mathrm{CH}_{2} \mathrm{Cl}_{2}(12.1 \mathrm{~mL})$ 
was added a solution of isobutyl phenyl ketene (50.0 mg, $0.29 \mathrm{mmol})$ in $\mathrm{CH}_{2} \mathrm{Cl}_{2}(1.9 \mathrm{~mL})$ over approximately $1 \mathrm{~min}$; the syringe was rinsed with $\mathrm{CH}_{2} \mathrm{Cl}_{2}(1.1 \mathrm{~mL})$. The resulting solution was stirred for $12 \mathrm{~h}$. The solvent was then removed by evaporation, and the residue was purified by column chromatography $(2.5 \rightarrow 10 \%$ EtOAc/hexanes), which furnished the $\beta$-lactam as a colorless oil $(87.8 \mathrm{mg}, 74 \%)$.

Second run (same scale): Yield: $83.0 \mathrm{mg}(70 \%)$.

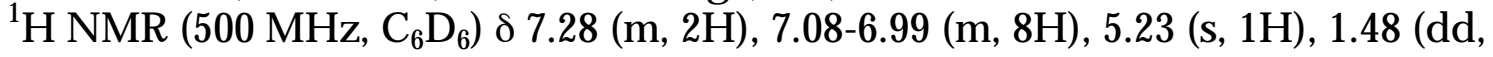
$\mathrm{J}=7.5, \mathrm{~J}=6.5 \mathrm{~Hz}, 1 \mathrm{H}), 1.28(\mathrm{~m}, 1 \mathrm{H}), 1.17(\mathrm{dd}, \mathrm{J}=4.5, \mathrm{~J}=10.0 \mathrm{~Hz}, 1 \mathrm{H}), 0.66(\mathrm{~d}, \mathrm{~J}=6.5 \mathrm{~Hz}, 3 \mathrm{H})$, $0.22(\mathrm{~d}, \mathrm{~J}=7.0 \mathrm{~Hz}, 3 \mathrm{H}) .{ }^{13} \mathrm{C}$ NMR $\left(126 \mathrm{MHz}, \mathrm{C}_{6} \mathrm{D}_{6}\right) \delta 167.6,138.5,133.4,129.9,129.8,129.3$, 128.7, 127.9, 126.5, $120.5\left(\mathrm{q},{ }^{1} \mathrm{~J}_{\mathrm{CF}}=277 \mathrm{~Hz}\right), 72.1,69.9,42.7,25.0,24.2,23.3 .{ }^{19} \mathrm{~F}$ NMR $(282$ MHz, $\mathrm{C}_{6} \mathrm{D}_{6}$ ) $\delta$-75.9. FTIR (NaCl) 1816, 1413, 1266, $739 \mathrm{~cm}^{-1}$. HRMS (ESI, m/e) calcd for $\mathrm{C}_{20} \mathrm{H}_{20} \mathrm{~F}_{3} \mathrm{NNaO}_{3} \mathrm{~S}(\mathrm{M}+\mathrm{Na})$ 434.1116, found 434.1016. [ $\left.\alpha\right]^{20}{ }_{\mathrm{D}}-65$ (c $0.24, \mathrm{CH}_{2} \mathrm{Cl}_{2}, 63 \%$ ee).

For ee analysis, the $\beta$-lactam $(46.5 \mathrm{mg}, 0.11 \mathrm{mmol})$ was reduced to the alcohol $(1.0 \mathrm{M}$ $\mathrm{LiAlH}_{4}$ in THF; $1.9 \mathrm{~mL} ; 3.0$ equiv). Yield: $38.0 \mathrm{mg}(81 \%)$ of a waxy white oil. HPLC analysis: $63 \%$ ee [Daicel CHIRALCEL AD-H column; $1.0 \mathrm{~mL} / \mathrm{min}$; solvent system: $1.0 \%$ isopropanol/hexanes; retention times: $27.6 \mathrm{~min}$ (major), $25.4 \mathrm{~min}$ (minor)]. Second run: Yield: $38.0 \mathrm{mg}(81 \%), 63 \%$ ee.

${ }^{1} \mathrm{H}$ NMR $\left(500 \mathrm{MHz}, \mathrm{C}_{6} \mathrm{D}_{6}\right) \delta 7.31(\mathrm{~d}, \mathrm{~J}=7.5 \mathrm{~Hz}, 2 \mathrm{H}), 7.25(\mathrm{~m}, 4 \mathrm{H}), 7.16-7.08(\mathrm{~m}, 4 \mathrm{H})$, $4.99(\mathrm{~s}, 1 \mathrm{H}), 3.61(\mathrm{~d}, \mathrm{~J}=11.0 \mathrm{~Hz}, 1 \mathrm{H}), 3.23(\mathrm{~d}, \mathrm{~J}=11.0 \mathrm{~Hz}, 1 \mathrm{H}), 1.42(\mathrm{dd}, \mathrm{J}=9.5, \mathrm{~J}=4.5 \mathrm{~Hz}, 1 \mathrm{H})$, $1.26($ broad s, $1 \mathrm{H}), 1.00(\mathrm{~m}, 1 \mathrm{H}), 0.84(\mathrm{dd}, \mathrm{J}=8.0, \mathrm{~J}=6.0 \mathrm{~Hz}, 1 \mathrm{H}), 0.42($ broad s, $1 \mathrm{H}), 0.38(\mathrm{~d}$, $\mathrm{J}=6.5 \mathrm{~Hz}, 3 \mathrm{H}), 0.36(\mathrm{~d}, \mathrm{~J}=8.0 \mathrm{~Hz}, 3 \mathrm{H}) .{ }^{13} \mathrm{C}$ NMR $\left(126 \mathrm{MHz}^{\mathrm{C}} \mathrm{CDCl}_{3}\right) \delta 140.3,138.0,128.8$, $128.4,128.3,127.9,127.3,119.1\left(\mathrm{q},{ }^{1} \mathrm{~J}_{\mathrm{CF}}=321 \mathrm{~Hz}\right), 67.9,65.0,50.2,46.4,25.0,24.9,24.5 .{ }^{19} \mathrm{~F}$ $\operatorname{NMR}\left(282 \mathrm{MHz}, \mathrm{CDCl}_{3}\right) \delta-78.6$. FTIR (NaCl) 3605, 1423, 1266, 1199, 1229, $740 \mathrm{~cm}^{-1}$. HRMS (ESI, $\mathrm{m} / \mathrm{e}$ ) calcd for $\mathrm{C}_{20} \mathrm{H}_{24} \mathrm{~F}_{3} \mathrm{NNaO}_{3} \mathrm{~S}(\mathrm{M}+\mathrm{Na}) 438.1429$, found 438.1334 . $[\alpha]^{20}{ }_{\mathrm{D}}$ -1.0 (c $0.26, \mathrm{CH}_{2} \mathrm{Cl}_{2}, 63 \%$ ee).

Table 1, entry 4. A solution of 1,1,1-trifluoro- $N$-[(4-fluorophenyl)methylene]methanesulfonamide $(50.0 \mathrm{mg}, 0.20 \mathrm{mmol})$ and $(-)-1(7.4 \mathrm{mg}, 20 \mu \mathrm{mol})$ in a mixture of $\mathrm{CH}_{2} \mathrm{Cl}_{2}(4.6 \mathrm{~mL})$ and toluene $(4.3 \mathrm{~mL})$ was cooled to $0{ }^{\circ} \mathrm{C}$. To this solution was added a solution of phenyl methyl ketene $(25.9 \mathrm{mg}, 0.20 \mathrm{mmol})$ in toluene $(1.0 \mathrm{~mL})$ over approximately $1 \mathrm{~min}$; the syringe was rinsed with $\mathrm{CH}_{2} \mathrm{Cl}_{2}(0.7 \mathrm{~mL})$. The resulting solution was allowed to warm to room temperature and stirred for $12 \mathrm{~h}$. The solvent was then removed by evaporation, and the residue was purified by column chromatography (10\% EtOAc/hexanes), which furnished the $\beta$-lactam as a colorless oil (63.2 $\mathrm{mg}, 83 \%)$.

HPLC analysis: $87 \%$ ee [Daicel CHIRALCEL AD-H column; $1.0 \mathrm{~mL} / \mathrm{min}$; solvent system: 1.0\% isopropanol/hexanes; retention times: $7.68 \mathrm{~min}$ (major), $6.72 \mathrm{~min}$ (minor)].

Second run (same scale): Yield: $64.0 \mathrm{mg}(84 \%), 83 \%$ ee.

${ }^{1} \mathrm{H}$ NMR $\left(500 \mathrm{MHz}, \mathrm{C}_{6} \mathrm{D}_{6}\right) \delta 7.15(\mathrm{~m}, 2 \mathrm{H}), 7.06(\mathrm{~m}, 3 \mathrm{H}), 6.69(\mathrm{~m}, 4 \mathrm{H}), 5.13(\mathrm{~s}, 1 \mathrm{H}), 0.78$ $(\mathrm{s}, 3 \mathrm{H}) .{ }^{13} \mathrm{C}$ NMR $\left(126 \mathrm{MHz}, \mathrm{CDCl}_{3}\right) \delta 168.1,163.4\left(\mathrm{~d},{ }^{1} \mathrm{~J}_{\mathrm{CF}}=250 \mathrm{~Hz}\right), 139.0,129.8,128.9(\mathrm{~d}$, $\left.{ }^{3} \mathrm{~J}_{\mathrm{CF}}=8.7 \mathrm{~Hz}\right), 128.7,125.3,119.5\left(\mathrm{q},{ }^{1} \mathrm{~J}_{\mathrm{CF}}=324 \mathrm{~Hz}\right), 116.4\left(\mathrm{~d},{ }^{2} \mathrm{~J}_{\mathrm{CF}}=21.9 \mathrm{~Hz}\right), 70.7,65.9,20.4$. ${ }^{19} \mathrm{~F}$ NMR $\left(282 \mathrm{MHz}, \mathrm{C}_{6} \mathrm{D}_{6}\right) \delta-76.2,-112.3$. FTIR $(\mathrm{NaCl}) 1822,1414,1213 \mathrm{~cm}^{-1}$. HRMS (ESI, m/e) calcd for $\mathrm{C}_{17} \mathrm{H}_{13} \mathrm{~F}_{4} \mathrm{NNaO}_{3} \mathrm{~S}(\mathrm{M}+\mathrm{Na})$ 410.0552, found 410.0460. $[\alpha]^{20}{ }_{\mathrm{D}}-106(c$ $0.33, \mathrm{CH}_{2} \mathrm{Cl}_{2}, 87 \%$ ee). 
Table 1, entry 5. A solution of 1,1,1-trifluoro- $\mathrm{N}$-[(4-trifluoromethylphenyl)methylene]methanesulfonamide $(60.0 \mathrm{mg}, 0.20 \mathrm{mmol})$ and $(-)-\mathbf{1}(7.4 \mathrm{mg}, 20 \mu \mathrm{mol})$ in a mixture of $\mathrm{CH}_{2} \mathrm{Cl}_{2}(4.6 \mathrm{~mL})$ and toluene $(4.2 \mathrm{~mL})$ was cooled to $0{ }^{\circ} \mathrm{C}$. To this solution was added a solution of phenyl methyl ketene $(26.0 \mathrm{mg}, 0.20 \mathrm{mmol})$ in toluene $(1.0 \mathrm{~mL})$ over approximately $1 \mathrm{~min}$; the syringe was rinsed with $\mathrm{CH}_{2} \mathrm{Cl}_{2}(0.6 \mathrm{~mL})$. The resulting solution was allowed to warm to room temperature and stirred for $12 \mathrm{~h}$. The solvent was then removed by evaporation, and the residue was purified by column chromatography (2.5\% EtOAc/hexanes), which furnished the $\beta$-lactam as a colorless oil (70.8 mg, 82\%).

HPLC analysis: $68 \%$ ee [Daicel CHIRALCEL AD-H column; $1.0 \mathrm{~mL} / \mathrm{min}$; solvent system: $1.0 \%$ isopropanol/hexanes; retention times: $6.63 \mathrm{~min}$ (major), $6.06 \mathrm{~min}$ (minor)]. The enantiomeric excess of the $\beta$-lactam can be improved by crystallization from pentane: the racemic $\beta$-lactam preferentially crystallizes, leaving enantiomerically enriched $\beta$-lactam in the mother liquor: $54.9 \mathrm{mg}(64 \%), 89 \%$ ee.

Second run (same scale): Yield: $66.0 \mathrm{mg}(77 \%), 70 \%$ ee. Crystallization as above yields $56.2 \mathrm{mg}(65 \%)$ of the $\beta$-lactam with $84 \%$ ee.

${ }^{1} \mathrm{H}$ NMR (500 MHz, $\left.\mathrm{C}_{6} \mathrm{D}_{6}\right) \delta 7.27(\mathrm{~d}, \mathrm{~J}=8.5 \mathrm{~Hz}, 2 \mathrm{H}), 7.13(\mathrm{~m}, 2 \mathrm{H}), 7.06(\mathrm{~m}, 3 \mathrm{H}), 6.78(\mathrm{~d}$, $\mathrm{J}=8.0 \mathrm{~Hz}, 2 \mathrm{H}), 5.12(\mathrm{~s}, 1 \mathrm{H}), 0.70(\mathrm{~s}, 3 \mathrm{H}) .{ }^{13} \mathrm{C}$ NMR $\left(126 \mathrm{MHz}, \mathrm{CDCl}_{3}\right) \delta 167.7,138.6,136.9$, $131.9\left(\mathrm{q},{ }^{2} \mathrm{~J}_{\mathrm{CF}}=32.8 \mathrm{~Hz}\right), 129.9,128.9,127.5,126.3\left(\mathrm{q},{ }^{3} \mathrm{~J}_{\mathrm{CF}}=4.0 \mathrm{~Hz}\right), 125.3,123.9\left(\mathrm{q},{ }^{1} \mathrm{~J}_{\mathrm{CF}}=272\right.$ $\mathrm{Hz}), 119.5\left(\mathrm{q},{ }^{1} \mathrm{~J}_{\mathrm{CF}}=324 \mathrm{~Hz}\right), 70.5,66.2,20.3 .{ }^{19} \mathrm{~F}$ NMR $\left(282 \mathrm{MHz}, \mathrm{C}_{6} \mathrm{D}_{6}\right) \delta-63.1,-76.1$. FTIR (NaCl) 1825, 1420, 1266, $740 \mathrm{~cm}^{-1}$. HRMS (ESI, m/e) calcd for $\mathrm{C}_{18} \mathrm{H}_{13} \mathrm{~F}_{6} \mathrm{NNaO}_{3} \mathrm{~S}$ $(\mathrm{M}+\mathrm{Na}) 460.0520$, found 460.0423 . $[\alpha]^{20}{ }_{\mathrm{D}}-87\left(\mathrm{c} 0.30, \mathrm{CH}_{2} \mathrm{Cl}_{2}, 89 \%\right.$ ee $)$.

Table 1, entry 6. A solution of 1,1,1-trifluoro- $N$-(4-methoxyphenylmethylene)methanesulfonamide $(50.0 \mathrm{mg}, 0.19 \mathrm{mmol})$ and $(-)-\mathbf{1}(7.0 \mathrm{mg}, 19 \mu \mathrm{mol})$ in toluene $(8.5$ $\mathrm{mL}$ ) was cooled to $0{ }^{\circ} \mathrm{C}$. To this solution was added a solution of phenyl methyl ketene $(24.7 \mathrm{mg}, 0.19 \mathrm{mmol})$ in toluene $(0.9 \mathrm{~mL})$ over approximately $1 \mathrm{~min}$; the syringe was rinsed with toluene $(0.4 \mathrm{~mL})$. The resulting solution was allowed to warm to room temperature and stirred for $12 \mathrm{~h}$. The solvent was then removed by evaporation, and the residue was purified by column chromatography (2.5\% EtOAc/hexanes), which furnished the $\beta$-lactam as a pale-yellow oil ( $56.0 \mathrm{mg}, 75 \%$; mixture of diastereomers).

HPLC analysis: $81 \%$ ee [Daicel CHIRALCEL AS-H column; $0.5 \mathrm{~mL} / \mathrm{min}$; solvent system: $1.0 \%$ isopropanol/hexanes; retention times: $16.8 \mathrm{~min}$ (major), $18.0 \mathrm{~min}$ (minor)].

Second run (same scale): Yield: $57.6 \mathrm{mg}(77 \%), 83 \%$ ee.

${ }^{1} \mathrm{H}$ NMR $\left(500 \mathrm{MHz}, \mathrm{C}_{6} \mathrm{D}_{6}\right) \delta 7.23(\mathrm{~m}, 2 \mathrm{H}), 7.06(\mathrm{~m}, 2 \mathrm{H}), 7.02(\mathrm{~m}, 1 \mathrm{H}), 6.88(\mathrm{~m}, 2 \mathrm{H})$, $6.64(\mathrm{~m}, 2 \mathrm{H}), 5.24(\mathrm{~s}, 1 \mathrm{H}), 3.24(\mathrm{~s}, 3 \mathrm{H}), 0.92(\mathrm{~s}, 3 \mathrm{H}) .{ }^{13} \mathrm{C} \mathrm{NMR}\left(126 \mathrm{MHz}, \mathrm{CDCl}_{3}\right) \delta$ 168.5, 160.6, 139.4, 129.7, 128.54, 128.46, 125.3, 124.5, $119.5\left(\mathrm{q},{ }_{\mathrm{CF}} \mathrm{J}_{\mathrm{CF}}=324 \mathrm{~Hz}\right), 114.6,71.3,65.8$, 55.5, 20.5. ${ }^{19} \mathrm{~F}$ NMR $\left(282 \mathrm{MHz}, \mathrm{C}_{6} \mathrm{D}_{6}\right) \delta$-75.9. FTIR (NaCl) 3057, 1819, 1412, 1265, 740 $\mathrm{cm}^{-1}$. HRMS (ESI, m/e) calcd for $\mathrm{C}_{18} \mathrm{H}_{16} \mathrm{~F}_{3} \mathrm{NNaO}_{4} \mathrm{~S}(\mathrm{M}+\mathrm{Na}) 422.0644$, found 422.0652 .

Table 1, entry 7. A solution of 1,1,1-trifluoro- $N$-(2-methylphenylmethylene)methanesulfonamide $(55.8 \mathrm{mg}, 0.22 \mathrm{mmol})$ and $(-)-1(8.4 \mathrm{mg}, 22 \mu \mathrm{mol})$ in toluene (10 $\mathrm{mL}$ ) was cooled to $0{ }^{\circ} \mathrm{C}$. To this solution was added a solution of phenyl methyl ketene $(29.3 \mathrm{mg}, 0.22 \mathrm{mmol})$ in toluene $(1.1 \mathrm{~mL})$ over approximately $2 \mathrm{~min}$; the syringe was rinsed with toluene $(0.6 \mathrm{~mL})$. The resulting solution was allowed to warm to room 
temperature and stirred for $12 \mathrm{~h}$. The solvent was then removed by evaporation, and the residue was purified by column chromatography (5\% EtOAc/hexanes), which furnished the $\beta$-lactam as a colorless oil $(75.7 \mathrm{mg}, 89 \%$; mixture of diastereomers).

The major diastereomer was isolated by column chromatography $(2.5 \rightarrow 5 \%$ EtOAc/hexanes) as a colorless oil. Yield: $52.1 \mathrm{mg}$ (61\%). HPLC analysis: $>99 \%$ ee [Daicel CHIRALCEL AD-H column; $1.0 \mathrm{~mL} / \mathrm{min}$; solvent system: 1.0\% isopropanol/hexanes; retention times: $13.7 \mathrm{~min}$ (major), $15.8 \mathrm{~min}$ (minor)].

Second run (same scale): Yield: $75.6 \mathrm{mg}$ (89\%). Yield of the major diastereomer: 50.2 $\operatorname{mg}(59 \%),>99 \%$ ee.

${ }^{1} \mathrm{H}$ NMR $\left(500 \mathrm{MHz}, \mathrm{C}_{6} \mathrm{D}_{6}\right) \delta 7.32(\mathrm{~m}, 1 \mathrm{H}), 7.19(\mathrm{~m}, 2 \mathrm{H}), 6.94-7.05(\mathrm{~m}, 5 \mathrm{H}), 6.79(\mathrm{~m}$, 1H), $5.58(\mathrm{~s}, 1 \mathrm{H}), 1.64(\mathrm{~s}, 3 \mathrm{H}), 0.88(\mathrm{~s}, 3 \mathrm{H}) .{ }^{13} \mathrm{C} \mathrm{NMR}\left(126 \mathrm{MHz} \mathrm{CDCl}_{3}\right) \delta$ 169.0, 138.4, $135.9,131.2,129.7,129.3,128.9,126.5,126.4,125.5,119.6\left(q,{ }^{1} J_{C F}=324 \mathrm{~Hz}\right), 69.5,65.5,19.9$, 17.5. ${ }^{19} \mathrm{~F}$ NMR $\left(282 \mathrm{MHz}, \mathrm{C}_{6} \mathrm{D}_{6}\right) \delta-76.3$. FTIR $(\mathrm{NaCl}) 1820,1414,1266,740 \mathrm{~cm}^{-1}$. HRMS (ESI, $\mathrm{m} / \mathrm{e}$ ) calcd for $\mathrm{C}_{18} \mathrm{H}_{16} \mathrm{~F}_{3} \mathrm{NNaO}_{3} \mathrm{~S}(\mathrm{M}+\mathrm{Na})$ 406.0803, found 406.0681. $[\alpha]_{\mathrm{D}}^{20}-135(\mathrm{c}$ $0.31, \mathrm{CH}_{2} \mathrm{Cl}_{2},>99 \%$ ee).

Table 1, entry 8. A solution of 1,1,1-trifluoro- $N$-[(2-bromophenyl)methylene]methanesulfonamide $(50.0 \mathrm{mg}, 0.16 \mathrm{mmol})$ and $(-)-1(5.9 \mathrm{mg}, 16 \mu \mathrm{mol})$ in a mixture of $\mathrm{CH}_{2} \mathrm{Cl}_{2}(3.7 \mathrm{~mL})$ and toluene $(3.4 \mathrm{~mL})$ was cooled to $0{ }^{\circ} \mathrm{C}$. To this solution was added a solution of phenyl methyl ketene $(20.9 \mathrm{mg}, 0.16 \mathrm{mmol})$ in toluene $(0.8 \mathrm{~mL})$ over approximately 2 min; the syringe was rinsed with $\mathrm{CH}_{2} \mathrm{Cl}_{2}(0.5 \mathrm{~mL})$. The resulting solution was allowed to warm to room temperature and stirred for $12 \mathrm{~h}$. The solvent was then removed by evaporation, and the residue was purified by column chromatography (2.5\% EtOAc/hexanes), which furnished the $\beta$-lactam as a colorless oil. (53.6 mg, 76\%; mixture of diastereomers).

The major diastereomer was isolated by column chromatography $(2.5 \rightarrow 5 \%$ EtOAc/hexanes) as colorless oil. Yield: $22.8 \mathrm{mg}(32 \%)$. GC analysis: 85\% ee [CPChirasil-Dex CB column; $175^{\circ} \mathrm{C}(5 \mathrm{~min}), 0.25^{\circ} \mathrm{C} / \mathrm{min}$ to $195{ }^{\circ} \mathrm{C} ; 9.31$ psi He; retention times: $39.8 \mathrm{~min}$ (major), $40.3 \mathrm{~min}$ (minor)].

Second run (same scale): Yield: $58.1 \mathrm{mg}(82 \%)$. Yield of the major diastereomer: 20.6 mg $(29 \%), 83 \%$ ee.

${ }^{1} \mathrm{H} \mathrm{NMR}\left(500 \mathrm{MHz}, \mathrm{C}_{6} \mathrm{D}_{6}\right) \delta 7.45(\mathrm{~m}, 2 \mathrm{H}), 7.23(\mathrm{dd}, \mathrm{J}=6.0, \mathrm{~J}=1.5 \mathrm{~Hz}, 1 \mathrm{H}), 7.11(\mathrm{~m}, 3 \mathrm{H})$, $7.03(\mathrm{~m}, 1 \mathrm{H}), 6.81(\mathrm{~m}, 1 \mathrm{H}), 6.58(\mathrm{td}, \mathrm{J}=6.5, \mathrm{~J}=1.5 \mathrm{~Hz}, 1 \mathrm{H})(\mathrm{m}, 4 \mathrm{H}), 5.95(\mathrm{~s}, 1 \mathrm{H}), 0.90(\mathrm{~s}, 3 \mathrm{H})$. ${ }^{13} \mathrm{C}$ NMR $\left(126 \mathrm{MHz}, \mathrm{CDCl}_{3}\right) \delta 168.5,137.9,133.8,133.1,130.9,129.3,128.9,128.4,128.0$, 126.1, 123.1, $119.6\left(\mathrm{q},{ }^{1} \mathrm{~J}_{\mathrm{CF}}=323 \mathrm{~Hz}\right), 70.4,66.5,18.1 .{ }^{19} \mathrm{~F} \mathrm{NMR}\left(282 \mathrm{MHz}, \mathrm{C}_{6} \mathrm{D}_{6}\right) \delta-76.2$. FTIR (NaCl) 1823, 1415, 1266, $740 \mathrm{~cm}^{-1}$. HRMS (ESI, m/e) calcd for $\mathrm{C}_{17} \mathrm{H}_{13} \mathrm{BrF}_{3} \mathrm{NNaO}_{3} \mathrm{~S}$ $(\mathrm{M}+\mathrm{Na}) 469.9752$, found 469.9650. $[\alpha]^{20}{ }_{\mathrm{D}}-138\left(c 0.30, \mathrm{CH}_{2} \mathrm{Cl}_{2}, 85 \%\right.$ ee).

Table 1, entry 9. A solution of 1,1,1-trifluoro- $N$-(2-naphthylmethylene)methanesulfonamide $(50.0 \mathrm{mg}, 0.17 \mathrm{mmol})$ and (-) -1 (6.5 $\mathrm{mg}, 17 \mu \mathrm{mol})$ in $\mathrm{CH}_{2} \mathrm{Cl}_{2}(8.0 \mathrm{~mL})$ was cooled to $0{ }^{\circ} \mathrm{C}$. To this solution was added a solution of phenyl methyl ketene $(23.0 \mathrm{mg}$, $0.17 \mathrm{mmol})$ in $\mathrm{CH}_{2} \mathrm{Cl}_{2}(0.9 \mathrm{~mL})$ over approximately $2 \mathrm{~min}$; the syringe was rinsed with $\mathrm{CH}_{2} \mathrm{Cl}_{2}(0.3 \mathrm{~mL})$. The resulting solution was allowed to warm to room temperature and stirred for $12 \mathrm{~h}$. The solvent was then removed by evaporation, and the residue was purified by column chromatography (10\% EtOAc/hexanes), which furnished the $\beta$ lactam as a colorless oil (56.0 $\mathrm{mg}, 77 \%)$. 
HPLC analysis: 93\% ee [Daicel CHIRALCEL OD-H column; $0.5 \mathrm{~mL} / \mathrm{min}$; solvent system: 1.0\% isopropanol/hexanes; retention times: $15.0 \mathrm{~min}$ (major), $21.0 \mathrm{~min}$ (minor)].

Second run (same scale): Yield: $54.3 \mathrm{mg}(74 \%), 94 \%$ ee.

${ }^{1} \mathrm{H}$ NMR $\left(500 \mathrm{MHz}, \mathrm{C}_{6} \mathrm{D}_{6}\right)$ d 7.59-7.49 (m, 4H), 7.27-7.24 (m, 3H), 7.13-7.04 (m, 5H), $5.44(\mathrm{~s}, 1 \mathrm{H}), 0.88(\mathrm{~s}, 3 \mathrm{H}) .{ }^{13} \mathrm{C} \mathrm{NMR}\left(126 \mathrm{MHz}, \mathrm{CDCl}_{3}\right) \delta$ 168.3, 139.3, 133.8, 133.2, 130.2, $129.8,129.2,128.7,128.3,128.1,127.3,127.28,126.9,125.4,123.8,119.6\left(\mathrm{q},{ }^{1} \mathrm{~J}_{\mathrm{CF}}=323 \mathrm{~Hz}\right)$, 71.5, 66.1, 20.4. ${ }^{19} \mathrm{~F}$ NMR $\left(282 \mathrm{MHz}, \mathrm{C}_{6} \mathrm{D}_{6}\right) \delta-76.2$. FTIR $(\mathrm{NaCl}) 1821,1413,1215,739 \mathrm{~cm}$ 1. HRMS (ESI, m/e) calcd for $\mathrm{C}_{21} \mathrm{H}_{16} \mathrm{~F}_{3} \mathrm{NNaO}_{3} \mathrm{~S}(\mathrm{M}+\mathrm{Na}) 442.0803$, found 442.0692. $[\alpha]^{20}{ }_{\mathrm{D}}$ -69 (c $0.29, \mathrm{CH}_{2} \mathrm{Cl}_{2}, 93 \%$ ee).

Table 1, entry 10. A solution of 1,1,1-trifluoro- $N$-(phenylmethylene)methanesulfonamide $(56.7 \mathrm{mg}, 0.24 \mathrm{mmol})$ and $(-)-\mathbf{1}(9.0 \mathrm{mg}, 24 \mu \mathrm{mol})$ in $\mathrm{CH}_{2} \mathrm{Cl}_{2}(11 \mathrm{~mL})$ was cooled to $0{ }^{\circ} \mathrm{C}$. To this solution was added a solution of diphenyl ketene $(46.4 \mathrm{mg}, 0.24 \mathrm{mmol})$ in $\mathrm{CH}_{2} \mathrm{Cl}_{2}(1.2 \mathrm{~mL})$ over approximately $3 \mathrm{~min}$; the syringe was rinsed with $\mathrm{CH}_{2} \mathrm{Cl}_{2}(0.4$ $\mathrm{mL}$ ). The resulting solution was allowed to warm to room temperature and stirred for $12 \mathrm{~h}$. The solvent was then removed by evaporation, and the residue was purified by column chromatography ( $10 \%$ EtOAc/hexanes), which furnished the $\beta$-lactam as a milky oil (60 mg, 60\%).

HPLC analysis: >99\% ee [Daicel CHIRALCEL OD column; $1.0 \mathrm{~mL} / \mathrm{min}$; solvent system: 5.0\% isopropanol/hexanes; retention times: $4.90 \mathrm{~min}$ (major), $5.74 \mathrm{~min}$ (minor)].

Second run (same scale): Yield: $65.0 \mathrm{mg}(63 \%), 97 \%$ ee.

${ }^{1} \mathrm{H}$ NMR $\left(500 \mathrm{MHz}, \mathrm{C}_{6} \mathrm{D}_{6}\right) \delta 7.46(\mathrm{~m}, 2 \mathrm{H}), 7.02(\mathrm{~m}, 2 \mathrm{H}), 6.96(\mathrm{~m}, 1 \mathrm{H}), 6.88-6.80(\mathrm{~m}$, 7H), $6.68(\mathrm{~m}, 3 \mathrm{H}), 5.95(\mathrm{~s}, 1 \mathrm{H}) .{ }^{13} \mathrm{C}$ NMR $\left(126 \mathrm{MHz}, \mathrm{CDCl}_{3}\right) \delta$ 166.4, 138.4, 135.4, 132.9, $129.5,128.7,128.63,128.56,128.01,127.97,127.8,126.9,119.3\left(\mathrm{q}^{1}{ }^{1} \mathrm{~J}_{\mathrm{CF}}=323 \mathrm{~Hz}\right), 74.9,70.7$. ${ }^{19} \mathrm{~F}$ NMR $\left(282 \mathrm{MHz}, \mathrm{C}_{6} \mathrm{D}_{6}\right) \delta-76.4$. FTIR (NaCl) 1814, 1414, 1266, $739 \mathrm{~cm}^{-1}$. HRMS (ESI, $\mathrm{m} / \mathrm{e}$ ) calcd for $\mathrm{C}_{22} \mathrm{H}_{16} \mathrm{~F}_{3} \mathrm{NNaO}_{3} \mathrm{~S}(\mathrm{M}+\mathrm{Na})$ 454.0803, found 454.0692. $[\alpha]^{20}{ }_{\mathrm{D}}-26(c 0.27$, $\mathrm{CH}_{2} \mathrm{Cl}_{2},>99 \%$ ee). 


\section{Derivatizations (eq 3-6) and Adduct A (eq 8)}

Eq 3. A solution of $\mathrm{LiAlH}_{4}$ (1.0 M in THF; $0.16 \mathrm{~mL} ; 3.0$ equiv) was added to a solution of the $\beta$-lactam $(20.0 \mathrm{mg}, 51.5 \mu \mathrm{mol} ; 87 \%$ ee) in THF $(0.7 \mathrm{~mL})$. The resulting mixture was heated to $50^{\circ} \mathrm{C}$ for $3 \mathrm{~h}$. Then, it was then cooled to $0{ }^{\circ} \mathrm{C}$, and the reaction was quenched by the addition of $1 \mathrm{~N} \mathrm{NaOH}(200 \mu \mathrm{L})$. The resulting slurry was dried over $\mathrm{MgSO}_{4}$ and filtered. The filtrate was concentrated, and the residue was purified by column chromatography $\left(40 \% \mathrm{Et}_{2} \mathrm{O} /\right.$ hexanes), which furnished the alcohol as a colorless film (15.9 mg, 79\%).

The ee of the product was determined by trifluoroacetylating (trifluoroacetic anhydride) the alcohol. GC analysis: $88 \%$ ee [CP-Chirasil-Dex CB column; $175{ }^{\circ} \mathrm{C}(5$ $\mathrm{min}$ ), $0.25^{\circ} \mathrm{C} / \mathrm{min}$ to $195^{\circ} \mathrm{C}$; $9.31 \mathrm{psi} \mathrm{He}$; retention times: $23.0 \mathrm{~min}$ (major), $22.7 \mathrm{~min}$ (minor)].

Second run (same scale): Yield: $15.0 \mathrm{mg}(74 \%), 88 \%$ ee.

${ }^{1} \mathrm{H} \mathrm{NMR}\left(500 \mathrm{MHz}, \mathrm{CDCl}_{3}\right) \delta 7.49(\mathrm{~m}, 2 \mathrm{H}), 7.43(\mathrm{~m}, 2 \mathrm{H}), 7.34(\mathrm{~m}, 1 \mathrm{H}), 7.26(\mathrm{~m}, 2 \mathrm{H})$, $7.05(\mathrm{~m}, 1 \mathrm{H}), 6.77(\mathrm{~d}, \mathrm{~J}=8.0 \mathrm{~Hz}, 1 \mathrm{H}), 5.01(\mathrm{~d}, \mathrm{~J}=8.0 \mathrm{~Hz}, 1 \mathrm{H}), 4.15(\mathrm{dd}, \mathrm{J}=8.0, \mathrm{~J}=3.0 \mathrm{~Hz}, 1 \mathrm{H})$, $3.79(\mathrm{dd}, \mathrm{J}=8.0, \mathrm{~J}=2.5 \mathrm{~Hz}, 1 \mathrm{H}), 2.01(\mathrm{~s}, 1 \mathrm{H}), 1.26(\mathrm{~s}, 1 \mathrm{H}), 1.16(\mathrm{~s}, 3 \mathrm{H}) .{ }^{13} \mathrm{C}$ NMR $(126 \mathrm{MHz}$, $\left.\mathrm{CDCl}_{3}\right) \delta 162.6\left(\mathrm{~d}, \mathrm{~J}_{\mathrm{CF}}=247 \mathrm{~Hz}\right), 141.8,130.0\left(\mathrm{~d},{ }^{3} \mathrm{~J}_{\mathrm{CF}}=8.1 \mathrm{~Hz}\right), 129.1,128.5,127.7,127.1$, $119.3\left(\mathrm{q}, \mathrm{J}_{\mathrm{CF}}=321 \mathrm{~Hz}\right), 115.4\left(\mathrm{~d},{ }^{2} \mathrm{~J}_{\mathrm{CF}}=21.9 \mathrm{~Hz}\right), 67.2,66.3,46.7$, 23.7. FTIR $(\mathrm{NaCl}) 3608$, $3055,1266,742 \mathrm{~cm}^{-1}$. HRMS (ESI, m/e) calcd for $\mathrm{C}_{17} \mathrm{H}_{17} \mathrm{~F}_{4} \mathrm{NNaO}_{3} \mathrm{~S}(\mathrm{M}+\mathrm{Na})$ 414.0757, found 414.0757. $[\alpha]^{20}{ }_{D}-1.45\left(c 0.75, \mathrm{CH}_{2} \mathrm{Cl}_{2}, 88 \%\right.$ ee).

Eq 4. A $1 \mathrm{~N}$ solution of $\mathrm{KOH}(374 \mu \mathrm{L}, 0.38 \mathrm{mmol})$ was added to a solution of the $\beta$ lactam $(72.4 \mathrm{mg}, 0.19 \mathrm{mmol} ; 79 \%$ ee) in ethanol $(1.5 \mathrm{~mL})$. The resulting mixture was heated to reflux for $2 \mathrm{~h}$ and then stirred at room temperature overnight. It was then cooled to $0{ }^{\circ} \mathrm{C}$, and the reaction was quenched by the addition of a solution of $1 \mathrm{~N} \mathrm{HCl}$ $(400 \mu \mathrm{L})$. The product was extracted with MTBE, and the organic layer was washed with saturated $\mathrm{NaHCO}_{3}$ and brine, dried over $\mathrm{Na}_{2} \mathrm{SO}_{4}$, and concentrated, furnishing a waxy white solid (68.4 $\mathrm{mg}, 90 \%)$.

The ee of the product was determined by reducing the acid to the alcohol (excess 1.0 $\mathrm{M} \mathrm{LiAlH}_{4}$ in THF at room temperature), and then trifluoroacetylating the alcohol (trifluoroacetic anhydride). GC analysis: 80\% ee [CP-Chirasil-Dex CB column; $175{ }^{\circ} \mathrm{C}(5$ $\mathrm{min}$ ), $0.25^{\circ} \mathrm{C} / \mathrm{min}$ to $195^{\circ} \mathrm{C}$; $9.31 \mathrm{psi} \mathrm{He}$; retention times: $23.0 \mathrm{~min}$ (major), $22.7 \mathrm{~min}$ (minor)].

Second run ( $\beta$-lactam: $30.0 \mathrm{mg}, 78 \%$ ee): Yield: $28.8 \mathrm{mg}(92 \%), 79 \%$ ee.

${ }^{1} \mathrm{H}$ NMR $\left(500 \mathrm{MHz}, \mathrm{C}_{6} \mathrm{D}_{6}\right) \delta 7.60(\mathrm{~m}, 4 \mathrm{H}), 7.38(\mathrm{~m}, 2 \mathrm{H}), 7.28(\mathrm{~m}, 1 \mathrm{H}), 7.10(\mathrm{~m}, 2 \mathrm{H})$, 4.97 (broad s, $1 \mathrm{H}), 3.27(\mathrm{~s}, 1 \mathrm{H}), 1.25(\mathrm{~s}, 3 \mathrm{H}) .{ }^{13} \mathrm{C} \mathrm{NMR}\left(126 \mathrm{MHz}, \mathrm{CDCl}_{3}\right) \delta 172.0,162.3$ $\left(\mathrm{d},{ }^{1} \mathrm{~J}_{\mathrm{CF}}=248 \mathrm{~Hz}\right), 142.0,133.3,129.6,128.6,127.6,127.0,119.2\left(\mathrm{q},{ }^{1} \mathrm{~J}_{\mathrm{CF}}=322 \mathrm{~Hz}\right), 114.9(\mathrm{~d}$, $\left.{ }^{2} \mathrm{~J}_{\mathrm{CF}}=22.4 \mathrm{~Hz}\right), 65.6,56.4,21.3 .{ }^{19} \mathrm{~F} \mathrm{NMR}\left(282 \mathrm{MHz}, \mathrm{CD}_{3} \mathrm{OD}\right) \delta-80.2,-117.2$. FTIR $(\mathrm{NaCl})$ $3574,1607,1397,1369,1266,1228,1203,740 \mathrm{~cm}^{-1}$. HRMS (ESI, m/e) calcd for $\mathrm{C}_{17} \mathrm{H}_{15} \mathrm{~F}_{4} \mathrm{NNaO}_{4} \mathrm{~S}(\mathrm{M}+\mathrm{Na}) 428.0658$, found 428.0550 . $[\alpha]^{20}{ }_{\mathrm{D}}+42\left(c 0.25, \mathrm{CH}_{2} \mathrm{Cl}_{2}, 79 \%\right.$ ee).

Eq 5. A solution of the $\beta$-lactam ( $21.0 \mathrm{mg}, 56.9 \mu \mathrm{mol} ; 79 \%$ ee) in THF $(0.7 \mathrm{~mL})$ was added to 2-bromobenzylamine $(11.6 \mathrm{mg}, 62.5 \mu \mathrm{mol})$. The reaction mixture was stirred at $40^{\circ} \mathrm{C}$ for $4 \mathrm{~h}$ and then at room temperature overnight. The resulting mixture was 
concentrated and purified by flash chromatography ( $5 \rightarrow 10 \%$ EtOAc/hexanes), which furnished $31.0 \mathrm{mg}(98 \%)$ of a white solid.

HPLC analysis: 79\% ee [Daicel CHIRALCEL AD-H column; $1.0 \mathrm{~mL} / \mathrm{min}$; solvent system: 10.0\% isopropanol/hexanes; retention times: $6.68 \mathrm{~min}$ (major), $10.7 \mathrm{~min}$ (minor)].

Second run (same scale; $\beta$-lactam: 83\% ee): Yield: $28.8 \mathrm{mg}(91 \%), 83 \%$ ee.

${ }^{1} \mathrm{H} \mathrm{NMR}\left(500 \mathrm{MHz}, \mathrm{CDCl}_{3}\right) \delta 8.53(\mathrm{~d}, \mathrm{~J}=8.5 \mathrm{~Hz}, 1 \mathrm{H}), 7.54(\mathrm{dd}, \mathrm{J}=7.5, \mathrm{~J}=1.0 \mathrm{~Hz}, 1 \mathrm{H})$, 7.41-7.20 (m, 11H), $6.35(\mathrm{~m}, 1 \mathrm{H}), 4.75(\mathrm{~d}, \mathrm{~J}=8.5 \mathrm{~Hz}, 1 \mathrm{H}), 4.63(\mathrm{dd}, \mathrm{J}=8.5, \mathrm{~J}=6.0 \mathrm{~Hz}, 1 \mathrm{H})$, $4.49(\mathrm{dd}, \mathrm{J}=9.0, \mathrm{~J}=6.0 \mathrm{~Hz}, 1 \mathrm{H}), 1.41(\mathrm{~s}, 3 \mathrm{H}) .{ }^{13} \mathrm{C} \mathrm{NMR}\left(126 \mathrm{MHz} \mathrm{CDCl}_{3}\right) \delta 175.0,141.4$, 137.7, 136.3, 133.1, 131.1, 129.8, 129.1, 128.6, 128.55, 128.48, 128.0, 127.9, 126.6, 124.0, 119.1 $\left(\mathrm{q},{ }^{1} \mathrm{~J}_{\mathrm{CF}}=321 \mathrm{~Hz}\right), 67.4,54.1,44.5,25.0$. FTIR $(\mathrm{NaCl}) 3456,3054,1649,1421,1265,739 \mathrm{~cm}^{-1}$. HRMS (ESI, $\mathrm{m} / \mathrm{e}$ ) calcd for $\mathrm{C}_{24} \mathrm{H}_{22} \mathrm{BrF}_{3} \mathrm{~N}_{2} \mathrm{NaO}_{3} \mathrm{~S}(\mathrm{M}+\mathrm{Na}) 577.0379$, found 577.0346. $[\alpha]^{20}-7.1$ (c $0.19, \mathrm{CH}_{2} \mathrm{Cl}_{2}, 79 \%$ ee). M.p. $166-167^{\circ} \mathrm{C}$.

Eq 6. Lithium metal $(4.40 \mathrm{mg}, 0.640 \mathrm{mmol})$ was combined with $\mathrm{TiCl}_{3}(14.1 \mathrm{mg}, 91.4$ $\mu \mathrm{mol})$ in THF $(1.2 \mathrm{~mL})$ in a Schlenk tube, and the resulting mixture was heated to reflux for $30 \mathrm{~min}$. The solution was allowed to cool, and then a solution of the $\beta$-lactam (20.0 $\mathrm{mg}, 45.7 \mu \mathrm{mol} ; 89 \%$ ee) in THF $(0.6 \mathrm{~mL})$ was added slowly. The resulting mixture was heated at $40{ }^{\circ} \mathrm{C}$ for $3 \mathrm{~h}$ and then at room temperature for $12 \mathrm{~h}$. The mixture was then cooled in an ice bath and quenched with $5 \% \mathrm{~K}_{2} \mathrm{CO}_{3}(1.0 \mathrm{~mL})$. A 1:1 mixture of $\mathrm{Et}_{2} \mathrm{O} / \mathrm{H}_{2} \mathrm{O}(1.0 \mathrm{~mL})$ was added, and then the reaction mixture was filtered through a pad of Celite. The organic layer was separated, washed with saturated $\mathrm{NaHCO}_{3}$ and brine, dried over $\mathrm{Na}_{2} \mathrm{SO}_{4}$, and concentrated. The product was purified by flash chromatography $\left(20 \rightarrow 50 \% \mathrm{Et}_{2} \mathrm{O} /\right.$ hexanes $)$. Yield: $11.9 \mathrm{mg}(85 \%)$ of a cloudy oil.

HPLC analysis: 90\% ee [Daicel CHIRALCEL AD-H column; $1.0 \mathrm{~mL} / \mathrm{min}$; solvent system: 10\% isopropanol/hexanes; retention times: 10.5 min (major), 8.85 min (minor)].

Second run (same scale; $\beta$-lactam: 89\% ee): Yield: $11.3 \mathrm{mg}(81 \%), 90 \%$ ee.

${ }^{1} \mathrm{H}$ NMR $\left(500 \mathrm{MHz}, \mathrm{C}_{6} \mathrm{D}_{6}\right) \delta 7.48(\mathrm{~m}, 2 \mathrm{H}), 7.33(\mathrm{~d}, \mathrm{~J}=8.5 \mathrm{~Hz}, 2 \mathrm{H}), 7.20(\mathrm{~m}, 2 \mathrm{H}), 7.10$ $(\mathrm{m}, 1 \mathrm{H}), 6.73(\mathrm{~d}, \mathrm{~J}=8.5 \mathrm{~Hz}, 2 \mathrm{H}), 5.08$ (broad s, $1 \mathrm{H}), 4.23(\mathrm{~s}, 1 \mathrm{H}), 0.91(\mathrm{~s}, 3 \mathrm{H}) .{ }^{13} \mathrm{C} \mathrm{NMR}$ $\left(126 \mathrm{MHz} \mathrm{CDCl}_{3}\right) \delta 172.4,141.6,141.3,130.7\left(\mathrm{q},{ }^{2} \mathrm{~J}_{\mathrm{CF}}=32.8 \mathrm{~Hz}\right), 129.2,127.7,127.0,126.02$, $125.98\left(\mathrm{q},{ }^{3} \mathrm{~J}_{\mathrm{CF}}=4.0 \mathrm{~Hz}\right), 123.3\left(\mathrm{q},{ }^{1} \mathrm{~J}_{\mathrm{CF}}=328 \mathrm{~Hz}\right), 64.7,63.4,20.0$. FTIR $(\mathrm{NaCl}) 3251,2927$, 1762. 1326. $740 \mathrm{~cm}^{-1}$. HRMS (ESI, m/e) calcd for $\mathrm{C}_{17} \mathrm{H}_{14} \mathrm{~F}_{3} \mathrm{NNaO}(\mathrm{M}+\mathrm{Na}) 328.0920$, found 328.0911. $[\alpha]^{20}-9.2\left(c 0.21, \mathrm{CH}_{2} \mathrm{Cl}_{2}, 90 \%\right.$ ee).

Eq 8. $N$-Benzylidenetrifluoromethanesulfonamide $(3.2 \mathrm{mg}, 13 \mathrm{mmol})$ and (-)-1 (5.0 $\mathrm{mg}, 13 \mathrm{mmol})$ were dissolved in $\mathrm{CD}_{2} \mathrm{Cl}_{2}(5.0 \mathrm{~mL})$.

${ }^{1} \mathrm{H} \mathrm{NMR}\left(400 \mathrm{MHz}, \mathrm{CD}_{2} \mathrm{Cl}_{2},-27^{\circ} \mathrm{C}\right) \delta 9.11(\mathrm{~d}, \mathrm{~J}=7.4 \mathrm{~Hz}, 1 \mathrm{H}), 7.18(\mathrm{~m}, 5 \mathrm{H}), 6.42(\mathrm{~s}$, $1 \mathrm{H}), 5.91(\mathrm{~d}, \mathrm{~J}=7.4 \mathrm{~Hz}, 1 \mathrm{H}), 4.55(\mathrm{~d}, \mathrm{~J}=2.3 \mathrm{~Hz}, 1 \mathrm{H}), 4.35$ (d, J=2.2 Hz, 1H), 3.99 (dd, J=5.8, $\mathrm{J}=2.9 \mathrm{~Hz}, 1 \mathrm{H}), 3.82(\mathrm{~m}, 2 \mathrm{H}), 3.68(\mathrm{~m}, 1 \mathrm{H}), 3.54(\mathrm{~m}, 1 \mathrm{H}), 2.19(\mathrm{~m}, 1 \mathrm{H}), 2.12(\mathrm{~m}, 3 \mathrm{H}), 1.67(\mathrm{~s}$, $15 \mathrm{H}) .{ }^{13} \mathrm{C} \mathrm{NMR}\left(126 \mathrm{MHz}, \mathrm{CD}_{2} \mathrm{Cl}_{2}\right.$, r.t.) $\delta 128.9,128.3,126.7,119.6\left(\mathrm{q},{ }^{1} \mathrm{~J}_{\mathrm{CF}}=327 \mathrm{~Hz}\right), 93.7$, 81.4, 79.4, 77.2, 66.3, 64.6, 51.4, 26.7, 25.0, 10.1, 1.3. ${ }^{19} \mathrm{~F} \mathrm{NMR}\left(282 \mathrm{MHz}, \mathrm{CD}_{2} \mathrm{Cl}_{2}\right) \delta-79.4$. 


\section{X-ray Crystal Structure of Adduct A}

Crystals suitable for X-ray structural analysis were obtained by crystallizing adduct A from acetonitrile at $-30{ }^{\circ} \mathrm{C}$.

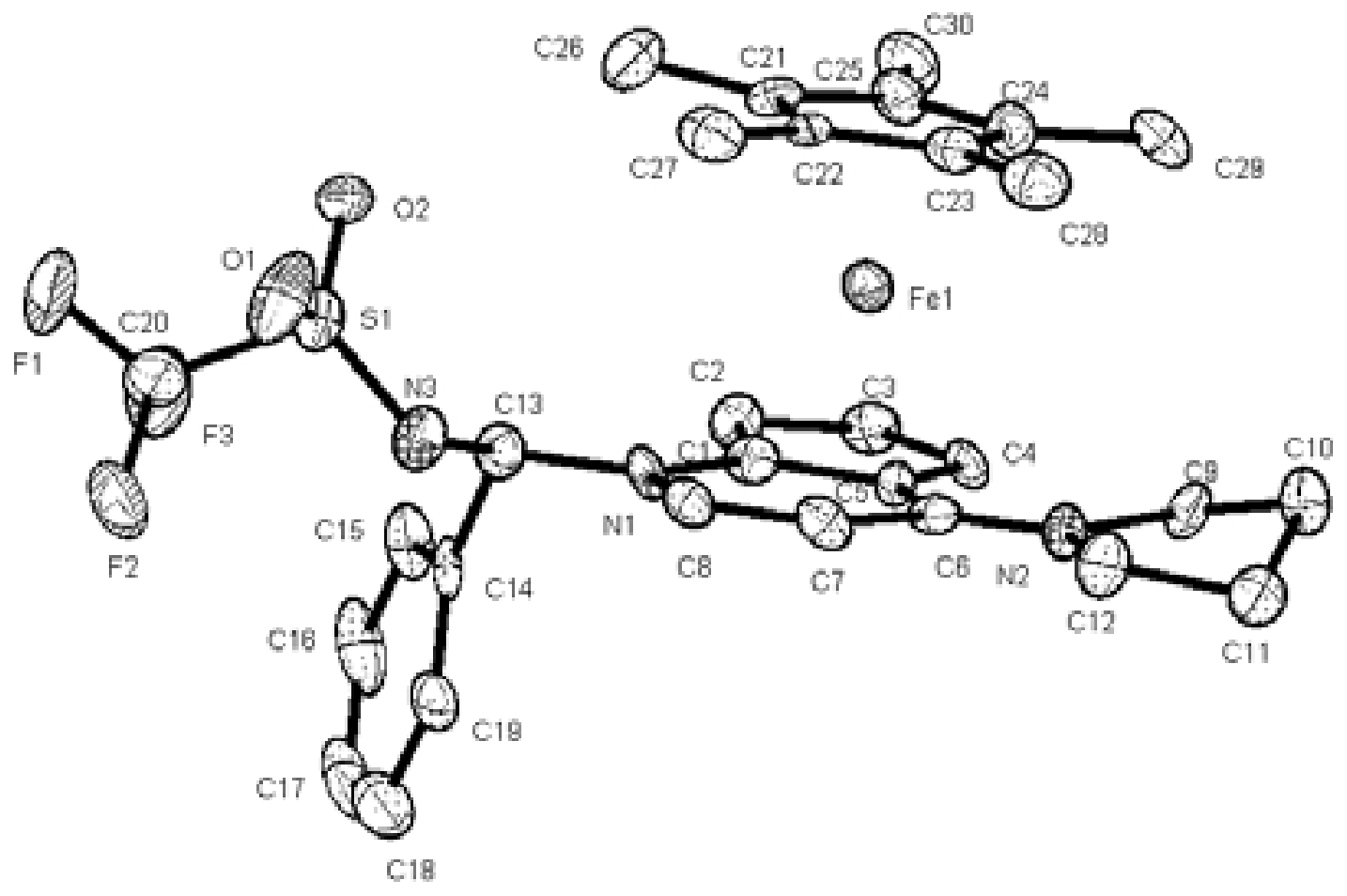


Table 1. Crystal data and structure refinement for $\mathbf{A}$.

Identification code

Empirical formula

Formula weight

Temperature

Wavelength

Crystal system

Space group

Unit cell dimensions

Volume

Z

Density (calculated)

Absorption coefficient

$\mathrm{F}(000)$

Crystal size

Theta range for data collection

Index ranges

Reflections collected

Independent reflections

Completeness to theta $=22.49^{\circ}$

Absorption correction

Max. and min. transmission

Refinement method

Data / restraints / parameters

Goodness-of-fit on F2

Final $\mathrm{R}$ indices [I $>2$ sigma(I)]

$\mathrm{R}$ indices (all data)

Absolute structure parameter

Largest diff. peak and hole
A

$\mathrm{C}_{33} \mathrm{H}_{38.5} \mathrm{~F}_{3} \mathrm{FeN}_{4.5} \mathrm{O}_{2} \mathrm{~S}$

675.09

100(2) K

$0.71073 \AA$

Monoclinic

P2(1)

$\mathrm{a}=12.9446(11) \AA \quad \quad=90^{\circ}$.

$\mathrm{b}=13.3261(12) \AA \quad \quad=94.617(3)^{\circ}$.

$\mathrm{c}=18.8459(16) \AA \quad \quad=90^{\circ}$.

3240.4(5) $\AA^{3}$

4

$1.384 \mathrm{Mg} / \mathrm{m}^{3}$

$0.584 \mathrm{~mm}^{-1}$

1412

$0.10 \times 0.10 \times 0.03 \mathrm{~mm}^{3}$

1.58 to $22.49^{\circ}$.

$-13<=\mathrm{h}<=13,-14<=\mathrm{k}<=14,0<=\mathrm{l}<=20$

34504

$8451[\mathrm{R}(\mathrm{int})=0.0992]$

$100.0 \%$

Semi-empirical from equivalents

0.9827 and 0.9439

Full-matrix least-squares on $\mathrm{F}^{2}$

8451 / 1 / 815

1.033

$\mathrm{R} 1=0.0485, \mathrm{wR} 2=0.0835$

$\mathrm{R} 1=0.0721, \mathrm{wR} 2=0.0923$

$0.014(17)$

0.419 and -0.266 e. $\AA^{-3}$ 
Table 2. Atomic coordinates ( $\times 10^{4}$ ) and equivalent isotropic displacement parameters $\left(\AA^{2} \times 10^{3}\right)$ for $\mathbf{A}$. $U(e q)$ is defined as one third of the trace of the orthogonalized $\mathrm{Uij}$ tensor.

\begin{tabular}{|c|c|c|c|c|}
\hline & $x$ & $\mathrm{y}$ & z & $\mathrm{U}(\mathrm{eq})$ \\
\hline $\mathrm{Fe}(1)$ & $-1138(1)$ & $-8011(1)$ & $-3462(1)$ & $20(1)$ \\
\hline$S(1)$ & $-5338(1)$ & $-9437(1)$ & $-4163(1)$ & $27(1)$ \\
\hline $\mathrm{F}(1)$ & $-6715(3)$ & $-10864(3)$ & $-3984(2)$ & $46(1)$ \\
\hline$F(2)$ & $-7008(3)$ & $-9486(3)$ & $-3462(2)$ & $71(1)$ \\
\hline$F(3)$ & $-5795(3)$ & $-10454(3)$ & $-3031(2)$ & $51(1)$ \\
\hline $\mathrm{O}(1)$ & $-5982(3)$ & $-9237(3)$ & $-4802(2)$ & $47(1)$ \\
\hline $\mathrm{O}(2)$ & $-4537(3)$ & $-10179(3)$ & $-4199(2)$ & $37(1)$ \\
\hline $\mathrm{N}(1)$ & $-3495(3)$ & $-7595(3)$ & $-3393(2)$ & $17(1)$ \\
\hline $\mathrm{N}(2)$ & $-1560(3)$ & $-5145(3)$ & $-3810(2)$ & 19(1) \\
\hline $\mathrm{N}(3)$ & $-5044(3)$ & $-8465(3)$ & $-3778(2)$ & $25(1)$ \\
\hline$C(1)$ & $-2481(4)$ & $-7537(4)$ & $-3054(3)$ & $20(1)$ \\
\hline$C(2)$ & $-1978(4)$ & $-8244(4)$ & $-2589(3)$ & $21(1)$ \\
\hline$C(3)$ & $-993(4)$ & $-7831(4)$ & $-2370(3)$ & $22(1)$ \\
\hline$C(4)$ & $-878(4)$ & $-6905(4)$ & $-2709(3)$ & $18(1)$ \\
\hline$C(5)$ & $-1805(4)$ & $-6698(4)$ & $-3156(3)$ & $16(1)$ \\
\hline$C(6)$ & $-2149(4)$ & $-5919(4)$ & $-3660(3)$ & $16(1)$ \\
\hline$C(7)$ & $-3164(4)$ & $-6042(4)$ & $-3991(3)$ & $21(1)$ \\
\hline$C(8)$ & $-3775(4)$ & $-6849(4)$ & $-3848(3)$ & $18(1)$ \\
\hline$C(9)$ & $-457(4)$ & $-4998(4)$ & $-3555(3)$ & $22(1)$ \\
\hline$C(10)$ & $-90(4)$ & $-4200(4)$ & $-4052(3)$ & $27(2)$ \\
\hline$C(11)$ & $-1058(4)$ & $-3568(4)$ & $-4224(3)$ & $26(2)$ \\
\hline$C(12)$ & $-1917(4)$ & $-4353(4)$ & $-4311(3)$ & $27(2)$ \\
\hline$C(13)$ & $-4170(4)$ & $-8465(4)$ & $-3246(3)$ & $22(1)$ \\
\hline$C(14)$ & $-4519(4)$ & $-8414(4)$ & $-2492(3)$ & $19(1)$ \\
\hline$C(15)$ & $-4200(4)$ & $-9159(5)$ & $-2003(3)$ & $33(2)$ \\
\hline$C(16)$ & $-4517(5)$ & $-9127(6)$ & $-1322(3)$ & $42(2)$ \\
\hline $\mathrm{C}(17)$ & $-5168(5)$ & $-8373(6)$ & $-1123(3)$ & $43(2)$ \\
\hline$C(18)$ & $-5504(5)$ & $-7652(5)$ & $-1617(3)$ & $39(2)$ \\
\hline$C(19)$ & $-5175(4)$ & $-7660(5)$ & $-2296(3)$ & $27(2)$ \\
\hline$C(20)$ & $-6251(5)$ & $-10085(5)$ & $-3636(4)$ & $38(2)$ \\
\hline
\end{tabular}




$\begin{array}{lrrrr}\mathrm{C}(21) & -1101(4) & -9360(4) & -3981(3) & 26(2) \\ \mathrm{C}(22) & -1470(4) & -8604(4) & -4461(3) & 23(2) \\ \mathrm{C}(23) & -690(4) & -7849(4) & -4482(3) & 24(2) \\ \mathrm{C}(24) & 169(4) & -8140(5) & -4001(3) & 24(1) \\ \mathrm{C}(25) & -84(4) & -9082(5) & -3694(3) & 25(2) \\ \mathrm{C}(26) & -1648(5) & -10331(5) & -3824(3) & 39(2) \\ \mathrm{C}(27) & -2489(4) & -8592(5) & -4922(3) & 35(2) \\ \mathrm{C}(28) & -748(5) & -6924(4) & -4950(3) & 31(2) \\ \mathrm{C}(29) & 1193(4) & -7598(5) & -3845(3) & 29(2) \\ \mathrm{C}(30) & 587(4) & -9696(5) & -3177(3) & 35(2) \\ \mathrm{Fe}(2) & -3227(1) & -4738(1) & -1139(1) & 22(1) \\ \mathrm{S}(101) & 696(1) & -3023(1) & -176(1) & 28(1) \\ \mathrm{F}(101) & 2715(2) & -3297(3) & -228(2) & 48(1) \\ \mathrm{F}(102) & 1893(3) & -4596(3) & 112(2) & 43(1) \\ \mathrm{F}(103) & 2185(2) & -3348(3) & 830(2) & 46(1) \\ \mathrm{O}(101) & 26(3) & -3370(3) & 345(2) & 31(1) \\ \mathrm{O}(102) & 961(3) & -1976(3) & -158(2) & 32(1) \\ \mathrm{N}(101) & -1051(3) & -4097(3) & -1582(2) & 22(1) \\ \mathrm{N}(102) & -3651(3) & -3475(3) & -3001(2) & 22(1) \\ \mathrm{N}(103) & 449(4) & -3371(3) & -945(2) & 26(1) \\ \mathrm{C}(101) & -1854(4) & -4795(5) & -1645(3) & 22(1) \\ \mathrm{C}(102) & -2001(4) & -5686(4) & -1273(3) & 21(1) \\ \mathrm{C}(103) & -2932(4) & -6132(4) & -1556(3) & 22(1) \\ \mathrm{C}(104) & -3408(4) & -5496(4) & -2083(3) & 23(2) \\ \mathrm{C}(105) & -2755(4) & -4642(4) & -2154(3) & 18(1) \\ \mathrm{C}(106) & -2833(4) & -3723(4) & -2553(3) & 21(1) \\ \mathrm{C}(107) & -1977(4) & -3056(5) & -2447(3) & 26(1) \\ \mathrm{C}(108) & -1136(4) & -3266(4) & -1979(3) & 25(2) \\ \mathrm{C}(109) & -4642(4) & -4045(4) & -3092(3) & 29(2) \\ \mathrm{C}(110) & -5392(5) & -3279(4) & -3448(3) & 39(2) \\ \mathrm{C}(111) & -4719(5) & -2659(5) & -3891(3) & 38(2) \\ \mathrm{C}(112) & -3729(5) & -2526(5) & -3406(3) & 33(2) \\ \mathrm{C}(113) & -94(4) & -4309(4) & -1074(3) & 23(1) \\ \mathrm{C}(114) & 579(4) & -5085(4) & -1400(3) & 23(2) \\ \mathrm{C}(115) & 1140(4) & -4825(5) & -1982(3) & 27(2) \\ \mathrm{C}(116) & & -5521(5) & -2266(3) & 32(2) \\ & & & \end{array}$




$\begin{array}{lrrrr}\mathrm{C}(117) & 1869(5) & -6483(5) & -1983(3) & 33(2) \\ \mathrm{C}(118) & 1332(5) & -6738(5) & -1411(3) & 32(2) \\ \mathrm{C}(119) & 679(4) & -6048(4) & -1121(3) & 24(2) \\ \mathrm{C}(120) & 1934(5) & -3595(5) & 148(3) & 33(2) \\ \mathrm{C}(121) & -3214(4) & -3516(4) & -477(3) & 25(2) \\ \mathrm{C}(122) & -3161(4) & -4409(4) & -74(3) & 23(1) \\ \mathrm{C}(123) & -4057(5) & -5005(4) & -282(3) & 29(2) \\ \mathrm{C}(124) & -4679(4) & -4456(5) & -807(3) & 31(2) \\ \mathrm{C}(125) & -4159(4) & -3538(5) & -942(3) & 27(2) \\ \mathrm{C}(126) & -2470(5) & -2646(4) & -429(3) & 31(2) \\ \mathrm{C}(127) & -2322(4) & -4686(5) & 495(3) & 35(2) \\ \mathrm{C}(128) & -4292(5) & -6005(5) & 34(3) & 47(2) \\ \mathrm{C}(129) & -5721(4) & -4773(6) & -1160(3) & 46(2) \\ \mathrm{C}(130) & -4544(4) & -2701(5) & -1426(3) & 36(2) \\ \mathrm{N}(15) & 983(6) & -12262(5) & -2878(4) & 74(2) \\ \mathrm{C}(1 S) & 1615(6) & -12158(5) & -2436(4) & 55(2) \\ \mathrm{C}(2 S) & 2368(6) & -12018(6) & -1839(4) & 64(2) \\ \mathrm{N}(2 S) & -6420(4) & -5717(5) & -3484(3) & 51(2) \\ \mathrm{C}(3 S) & -6521(5) & -6128(5) & -4021(4) & 35(2) \\ \mathrm{C}(4 S) & -6611(5) & -6644(5) & -4705(3) & 39(2) \\ \mathrm{N}(3 S) & -2049(5) & -10695(4) & -1949(3) & 56(2) \\ \mathrm{C}(5 S) & -1255(7) & -10604(5) & -1636(4) & 50(2) \\ \mathrm{C}(6 S) & -223(6) & -10463(6) & -1270(3) & 61(2)\end{array}$


Table 3. Bond lengths $[\AA]$ and angles $\left[{ }^{\circ}\right]$ for $\mathbf{A}$.

\begin{tabular}{ll}
\hline $\mathrm{Fe}(1)-\mathrm{C}(25)$ & $2.045(6)$ \\
$\mathrm{Fe}(1)-\mathrm{C}(21)$ & $2.049(6)$ \\
$\mathrm{Fe}(1)-\mathrm{C}(24)$ & $2.049(5)$ \\
$\mathrm{Fe}(1)-\mathrm{C}(22)$ & $2.054(5)$ \\
$\mathrm{Fe}(1)-\mathrm{C}(5)$ & $2.055(5)$ \\
$\mathrm{Fe}(1)-\mathrm{C}(4)$ & $2.056(5)$ \\
$\mathrm{Fe}(1)-\mathrm{C}(1)$ & $2.058(6)$ \\
$\mathrm{Fe}(1)-\mathrm{C}(23)$ & $2.062(5)$ \\
$\mathrm{Fe}(1)-\mathrm{C}(3)$ & $2.066(5)$ \\
$\mathrm{Fe}(1)-\mathrm{C}(2)$ & $2.068(5)$ \\
$\mathrm{S}(1)-\mathrm{O}(1)$ & $1.434(4)$ \\
$\mathrm{S}(1)-\mathrm{O}(2)$ & $1.439(4)$ \\
$\mathrm{S}(1)-\mathrm{N}(3)$ & $1.517(5)$ \\
$\mathrm{S}(1)-\mathrm{C}(20)$ & $1.822(7)$ \\
$\mathrm{F}(1)-\mathrm{C}(20)$ & $1.343(7)$ \\
$\mathrm{F}(2)-\mathrm{C}(20)$ & $1.325(7)$ \\
$\mathrm{F}(3)-\mathrm{C}(20)$ & $1.336(7)$ \\
$\mathrm{N}(1)-\mathrm{C}(8)$ & $1.344(6)$ \\
$\mathrm{N}(1)-\mathrm{C}(1)$ & $1.415(6)$ \\
$\mathrm{N}(1)-\mathrm{C}(13)$ & $1.491(7)$ \\
$\mathrm{N}(2)-\mathrm{C}(6)$ & $1.326(6)$ \\
$\mathrm{N}(2)-\mathrm{C}(12)$ & $1.466(6)$ \\
$\mathrm{N}(2)-\mathrm{C}(9)$ & $1.482(6)$ \\
$\mathrm{N}(3)-\mathrm{C}(13)$ & $1.451(6)$ \\
$\mathrm{C}(1)-\mathrm{C}(2)$ & $1.410(7)$ \\
$\mathrm{C}(1)-\mathrm{C}(5)$ & $1.443(7)$ \\
$\mathrm{C}(2)-\mathrm{C}(3)$ & $1.419(7)$ \\
$\mathrm{C}(3)-\mathrm{C}(4)$ & $1.403(7)$ \\
$\mathrm{C}(4)-\mathrm{C}(5)$ & $1.437(7)$ \\
$\mathrm{C}(5)-\mathrm{C}(6)$ & $1.452(7)$ \\
$\mathrm{C}(6)-\mathrm{C}(7)$ & $1.418(7)$ \\
$\mathrm{C}(7)-\mathrm{C}(8)$ & $1.374(7)$ \\
$\mathrm{C}(9)-\mathrm{C}(10)$ & $1.518(7)$ \\
$\mathrm{C}(10)-\mathrm{C}(11)$ & $1.523(7)$ \\
\end{tabular}




$\begin{array}{ll}\mathrm{C}(11)-\mathrm{C}(12) & 1.527(7) \\ \mathrm{C}(13)-\mathrm{C}(14) & 1.527(7) \\ \mathrm{C}(14)-\mathrm{C}(19) & 1.384(7) \\ \mathrm{C}(14)-\mathrm{C}(15) & 1.394(7) \\ \mathrm{C}(15)-\mathrm{C}(16) & 1.380(8) \\ \mathrm{C}(16)-\mathrm{C}(17) & 1.382(9) \\ \mathrm{C}(17)-\mathrm{C}(18) & 1.383(8) \\ \mathrm{C}(18)-\mathrm{C}(19) & 1.382(8) \\ \mathrm{C}(21)-\mathrm{C}(22) & 1.410(8) \\ \mathrm{C}(21)-\mathrm{C}(25) & 1.432(7) \\ \mathrm{C}(21)-\mathrm{C}(26) & 1.516(8) \\ \mathrm{C}(22)-\mathrm{C}(23) & 1.428(8) \\ \mathrm{C}(22)-\mathrm{C}(27) & 1.520(7) \\ \mathrm{C}(23)-\mathrm{C}(24) & 1.431(7) \\ \mathrm{C}(23)-\mathrm{C}(28) & 1.515(8) \\ \mathrm{C}(24)-\mathrm{C}(25) & 1.431(8) \\ \mathrm{C}(24)-\mathrm{C}(29) & 1.517(7) \\ \mathrm{C}(25)-\mathrm{C}(30) & 1.495(7) \\ \mathrm{Fe}(2)-\mathrm{C}(123) & 2.042(5) \\ \mathrm{Fe}(2)-\mathrm{C}(104) & 2.043(6) \\ \mathrm{Fe}(2)-\mathrm{C}(122) & 2.048(5) \\ \mathrm{Fe}(2)-\mathrm{C}(121) & 2.051(6) \\ \mathrm{Fe}(2)-\mathrm{C}(125) & 2.055(6) \\ \mathrm{Fe}(2)-\mathrm{C}(105) & 2.059(5) \\ \mathrm{Fe}(2)-\mathrm{C}(102) & 2.060(6) \\ \mathrm{Fe}(2)-\mathrm{C}(124) & 2.062(6) \\ \mathrm{Fe}(2)-\mathrm{C}(103) & 2.065(6) \\ \mathrm{Fe}(2)-\mathrm{C}(101) & 2.085(5) \\ \mathrm{S}(101)-\mathrm{O}(102) & 1.437(4) \\ \mathrm{S}(101)-\mathrm{O}(101) & 1.438(4) \\ \mathrm{S}(101)-\mathrm{N}(103) & 1.531(4) \\ \mathrm{S}(101)-\mathrm{C}(120) & 1.834(6) \\ \mathrm{F}(101)-\mathrm{C}(120) & 1.340(7) \\ \mathrm{F}(102)-\mathrm{C}(120) & 1.336(7) \\ \mathrm{F}(103)-\mathrm{C}(120) & 1.341(6) \\ \mathrm{N}(101)-\mathrm{C}(108) & 1.336(7) \\ & \end{array}$




$\begin{array}{ll}\mathrm{N}(101)-\mathrm{C}(101) & 1.394(7) \\ \mathrm{N}(101)-\mathrm{C}(113) & 1.529(6) \\ \mathrm{N}(102)-\mathrm{C}(106) & 1.341(6) \\ \mathrm{N}(102)-\mathrm{C}(112) & 1.477(7) \\ \mathrm{N}(102)-\mathrm{C}(109) & 1.488(7) \\ \mathrm{N}(103)-\mathrm{C}(113) & 1.445(7) \\ \mathrm{C}(101)-\mathrm{C}(102) & 1.398(8) \\ \mathrm{C}(101)-\mathrm{C}(105) & 1.463(7) \\ \mathrm{C}(102)-\mathrm{C}(103) & 1.410(7) \\ \mathrm{C}(103)-\mathrm{C}(104) & 1.408(7) \\ \mathrm{C}(104)-\mathrm{C}(105) & 1.431(8) \\ \mathrm{C}(105)-\mathrm{C}(106) & 1.437(7) \\ \mathrm{C}(106)-\mathrm{C}(107) & 1.423(7) \\ \mathrm{C}(107)-\mathrm{C}(108) & 1.374(7) \\ \mathrm{C}(109)-\mathrm{C}(110) & 1.526(7) \\ \mathrm{C}(110)-\mathrm{C}(111) & 1.502(8) \\ \mathrm{C}(111)-\mathrm{C}(112) & 1.523(7) \\ \mathrm{C}(113)-\mathrm{C}(114) & 1.513(8) \\ \mathrm{C}(114)-\mathrm{C}(119) & 1.389(7) \\ \mathrm{C}(114)-\mathrm{C}(115) & 1.407(7) \\ \mathrm{C}(115)-\mathrm{C}(116) & 1.389(8) \\ \mathrm{C}(116)-\mathrm{C}(117) & 1.389(8) \\ \mathrm{C}(117)-\mathrm{C}(118) & 1.372(8) \\ \mathrm{C}(118)-\mathrm{C}(119) & 1.390(8) \\ \mathrm{C}(121)-\mathrm{C}(122) & 1.410(8) \\ \mathrm{C}(121)-\mathrm{C}(125) & 1.446(8) \\ \mathrm{C}(121)-\mathrm{C}(126) & 1.505(8) \\ \mathrm{C}(122)-\mathrm{C}(123) & 1.434(8) \\ \mathrm{C}(122)-\mathrm{C}(127) & 1.509(7) \\ \mathrm{C}(123)-\mathrm{C}(124) & 1.425(8) \\ \mathrm{C}(123)-\mathrm{C}(128) & 1.501(8) \\ \mathrm{C}(124)-\mathrm{C}(125) & 1.429(8) \\ \mathrm{C}(124)-\mathrm{C}(129) & 1.516(8) \\ \mathrm{C}(125)-\mathrm{C}(130) & 1.500(8) \\ \mathrm{N}(1 \mathrm{~S})-\mathrm{C}(1 \mathrm{~S}) & 1.129(8) \\ \mathrm{C}(15)-\mathrm{C}(2 \mathrm{~S}) & 1.441(9) \\ & \end{array}$




$\begin{array}{lc}\text { N(2S)-C(3S) } & 1.150(8) \\ \text { C(3S)-C(4S) } & 1.456(9) \\ \text { N(3S)-C(5S) } & 1.150(8) \\ \text { C(5S)-C(6S) } & 1.465(10) \\ & \\ \text { C(25)-Fe(1)-C(21) } & 40.9(2) \\ \text { C(25)-Fe(1)-C(24) } & 40.9(2) \\ \text { C(21)-Fe(1)-C(24) } & 68.8(2) \\ \text { C(25)-Fe(1)-C(22) } & 68.3(2) \\ \text { C(21)-Fe(1)-C(22) } & 40.2(2) \\ \text { C(24)-Fe(1)-C(22) } & 68.6(2) \\ \text { C(25)-Fe(1)-C(5) } & 163.1(2) \\ \text { C(21)-Fe(1)-C(5) } & 155.2(2) \\ \text { C(24)-Fe(1)-C(5) } & 126.4(2) \\ \text { C(22)-Fe(1)-C(5) } & 121.5(2) \\ \text { C(25)-Fe(1)-C(4) } & 125.0(2) \\ \text { C(21)-Fe(1)-C(4) } & 161.9(2) \\ \text { C(24)-Fe(1)-C(4) } & 107.9(2) \\ \text { C(22)-Fe(1)-C(4) } & 156.8(2) \\ \text { C(5)-Fe(1)-C(4) } & 40.91(19) \\ \text { C(25)-Fe(1)-C(1) } & 153.2(2) \\ \text { C(21)-Fe(1)-C(1) } & 120.0(2) \\ \text { C(24)-Fe(1)-C(1) } & 165.4(2) \\ \text { C(22)-Fe(1)-C(1) } & 109.9(2) \\ \text { C(5)-Fe(1)-C(1) } & 41.1(2) \\ \text { C(4)-Fe(1)-C(1) } & 67.5(2) \\ \text { C(25)-Fe(1)-C(23) } & 68.4(2) \\ \text { C(21)-Fe(1)-C(23) } & 68.2(2) \\ \text { C(24)-Fe(1)-C(23) } & 40.7(2) \\ \text { C(22)-Fe(1)-C(23) } & 40.6(2) \\ \text { C(5)-Fe(1)-C(23) } & 109.2(2) \\ \text { C(4)-Fe(1)-C(23) } & 121.7(2) \\ \text { C(1)-Fe(1)-C(23) } & 128.7(2) \\ \text { C(25)-Fe(1)-C(3) } & 106.6(2) \\ \text { C(21)-Fe(1)-C(3) } & 125.0(2) \\ \text { C(24)-Fe(1)-C(3) } & 119.4(2) \\ & \end{array}$




$\begin{array}{lr}\mathrm{C}(22)-\mathrm{Fe}(1)-\mathrm{C}(3) & 162.3(2) \\ \mathrm{C}(5)-\mathrm{Fe}(1)-\mathrm{C}(3) & 68.1(2) \\ \mathrm{C}(4)-\mathrm{Fe}(1)-\mathrm{C}(3) & 39.8(2) \\ \mathrm{C}(1)-\mathrm{Fe}(1)-\mathrm{C}(3) & 66.6(2) \\ \mathrm{C}(23)-\mathrm{Fe}(1)-\mathrm{C}(3) & 155.0(2) \\ \mathrm{C}(25)-\mathrm{Fe}(1)-\mathrm{C}(2) & 117.9(2) \\ \mathrm{C}(21)-\mathrm{Fe}(1)-\mathrm{C}(2) & 106.4(2) \\ \mathrm{C}(24)-\mathrm{Fe}(1)-\mathrm{C}(2) & 152.8(2) \\ \mathrm{C}(22)-\mathrm{Fe}(1)-\mathrm{C}(2) & 125.9(2) \\ \mathrm{C}(5)-\mathrm{Fe}(1)-\mathrm{C}(2) & 69.2(2) \\ \mathrm{C}(4)-\mathrm{Fe}(1)-\mathrm{C}(2) & 68.0(2) \\ \mathrm{C}(1)-\mathrm{Fe}(1)-\mathrm{C}(2) & 40.0(2) \\ \mathrm{C}(23)-\mathrm{Fe}(1)-\mathrm{C}(2) & 164.2(2) \\ \mathrm{C}(3)-\mathrm{Fe}(1)-\mathrm{C}(2) & 40.16(19) \\ \mathrm{O}(1)-\mathrm{S}(1)-\mathrm{O}(2) & 117.5(3) \\ \mathrm{O}(1)-\mathrm{S}(1)-\mathrm{N}(3) & 110.4(3) \\ \mathrm{O}(2)-\mathrm{S}(1)-\mathrm{N}(3) & 117.1(2) \\ \mathrm{O}(1)-\mathrm{S}(1)-\mathrm{C}(20) & 100.8(3) \\ \mathrm{O}(2)-\mathrm{S}(1)-\mathrm{C}(20) & 101.6(3) \\ \mathrm{N}(3)-\mathrm{S}(1)-\mathrm{C}(20) & 107.0(3) \\ \mathrm{C}(8)-\mathrm{N}(1)-\mathrm{C}(1) & 116.1(5) \\ \mathrm{C}(8)-\mathrm{N}(1)-\mathrm{C}(13) & 124.2(4) \\ \mathrm{C}(1)-\mathrm{N}(1)-\mathrm{C}(13) & 119.7(4) \\ \mathrm{C}(6)-\mathrm{N}(2)-\mathrm{C}(12) & 122.7(4) \\ \mathrm{C}(6)-\mathrm{N}(2)-\mathrm{C}(9) & 126.0(4) \\ \mathrm{C}(12)-\mathrm{N}(2)-\mathrm{C}(9) & 111.1(4) \\ \mathrm{C}(13)-\mathrm{N}(3)-\mathrm{S}(1) & 118.8(4) \\ \mathrm{C}(2)-\mathrm{C}(1)-\mathrm{N}(1) & 127.5(5) \\ \mathrm{C}(2)-\mathrm{C}(1)-\mathrm{C}(5) & 110.3(5) \\ \mathrm{N}(1)-\mathrm{C}(1)-\mathrm{C}(5) & 122.2(5) \\ \mathrm{C}(2)-\mathrm{C}(1)-\mathrm{Fe}(1) & 70.4(3) \\ \mathrm{N}(1)-\mathrm{C}(1)-\mathrm{Fe}(1) & 69.6(3) \\ \mathrm{C}(5)-\mathrm{C}(1)-\mathrm{Fe}(1) & 69.8(3) \\ \mathrm{C}(1)-\mathrm{C}(2)-\mathrm{C}(3) & \\ \mathrm{C}(1)-\mathrm{C}(2)-\mathrm{Fe}(1) & \\ \mathrm{C}(3)-\mathrm{C}(2)-\mathrm{Fe}(1) & \end{array}$




$\begin{array}{lr}\mathrm{C}(4)-\mathrm{C}(3)-\mathrm{C}(2) & 109.6(5) \\ \mathrm{C}(4)-\mathrm{C}(3)-\mathrm{Fe}(1) & 69.7(3) \\ \mathrm{C}(2)-\mathrm{C}(3)-\mathrm{Fe}(1) & 70.0(3) \\ \mathrm{C}(3)-\mathrm{C}(4)-\mathrm{C}(5) & 108.7(5) \\ \mathrm{C}(3)-\mathrm{C}(4)-\mathrm{Fe}(1) & 70.5(3) \\ \mathrm{C}(5)-\mathrm{C}(4)-\mathrm{Fe}(1) & 69.5(3) \\ \mathrm{C}(4)-\mathrm{C}(5)-\mathrm{C}(1) & 105.1(5) \\ \mathrm{C}(4)-\mathrm{C}(5)-\mathrm{C}(6) & 135.9(5) \\ \mathrm{C}(1)-\mathrm{C}(5)-\mathrm{C}(6) & 118.9(5) \\ \mathrm{C}(4)-\mathrm{C}(5)-\mathrm{Fe}(1) & 69.6(3) \\ \mathrm{C}(1)-\mathrm{C}(5)-\mathrm{Fe}(1) & 69.6(3) \\ \mathrm{C}(6)-\mathrm{C}(5)-\mathrm{Fe}(1) & 122.6(4) \\ \mathrm{N}(2)-\mathrm{C}(6)-\mathrm{C}(7) & 121.6(5) \\ \mathrm{N}(2)-\mathrm{C}(6)-\mathrm{C}(5) & 123.0(5) \\ \mathrm{C}(7)-\mathrm{C}(6)-\mathrm{C}(5) & 115.4(5) \\ \mathrm{C}(8)-\mathrm{C}(7)-\mathrm{C}(6) & 122.2(5) \\ \mathrm{N}(1)-\mathrm{C}(8)-\mathrm{C}(7) & 125.0(5) \\ \mathrm{N}(2)-\mathrm{C}(9)-\mathrm{C}(10) & 103.5(4) \\ \mathrm{C}(9)-\mathrm{C}(10)-\mathrm{C}(11) & 103.1(4) \\ \mathrm{C}(10)-\mathrm{C}(11)-\mathrm{C}(12) & 102.9(5) \\ \mathrm{N}(2)-\mathrm{C}(12)-\mathrm{C}(11) & 103.6(4) \\ \mathrm{N}(3)-\mathrm{C}(13)-\mathrm{N}(1) & 107.9(4) \\ \mathrm{N}(3)-\mathrm{C}(13)-\mathrm{C}(14) & 111.8(4) \\ \mathrm{N}(1)-\mathrm{C}(13)-\mathrm{C}(14) & 111.1(4) \\ \mathrm{C}(19)-\mathrm{C}(14)-\mathrm{C}(15) & 119.8(5) \\ \mathrm{C}(19)-\mathrm{C}(14)-\mathrm{C}(13) & 120.9(5) \\ \mathrm{C}(15)-\mathrm{C}(14)-\mathrm{C}(13) & 119.3(5) \\ \mathrm{C}(16)-\mathrm{C}(15)-\mathrm{C}(14) & 119.9(6) \\ \mathrm{C}(15)-\mathrm{C}(16)-\mathrm{C}(17) & 120.5(6) \\ \mathrm{C}(16)-\mathrm{C}(17)-\mathrm{C}(18) & 119.3(6) \\ \mathrm{C}(19)-\mathrm{C}(18)-\mathrm{C}(17) & 120.9(6) \\ \mathrm{C}(18)-\mathrm{C}(19)-\mathrm{C}(14) & 119.6(6) \\ \mathrm{F}(2)-\mathrm{C}(20)-\mathrm{F}(3) & 107.2(6) \\ \mathrm{F}(2)-\mathrm{C}(20)-\mathrm{F}(1) & 106.0(5) \\ \mathrm{F}(3)-\mathrm{C}(20)-\mathrm{F}(1) & 106.4(5) \\ \mathrm{F}(2)-\mathrm{C}(20)-\mathrm{S}(1) & 112.0(5) \\ & \end{array}$




$\begin{array}{lr}\mathrm{F}(3)-\mathrm{C}(20)-\mathrm{S}(1) & 112.2(4) \\ \mathrm{F}(1)-\mathrm{C}(20)-\mathrm{S}(1) & 112.6(5) \\ \mathrm{C}(22)-\mathrm{C}(21)-\mathrm{C}(25) & 108.2(5) \\ \mathrm{C}(22)-\mathrm{C}(21)-\mathrm{C}(26) & 126.6(5) \\ \mathrm{C}(25)-\mathrm{C}(21)-\mathrm{C}(26) & 125.2(5) \\ \mathrm{C}(22)-\mathrm{C}(21)-\mathrm{Fe}(1) & 70.1(3) \\ \mathrm{C}(25)-\mathrm{C}(21)-\mathrm{Fe}(1) & 69.4(3) \\ \mathrm{C}(26)-\mathrm{C}(21)-\mathrm{Fe}(1) & 128.8(4) \\ \mathrm{C}(21)-\mathrm{C}(22)-\mathrm{C}(23) & 108.5(5) \\ \mathrm{C}(21)-\mathrm{C}(22)-\mathrm{C}(27) & 127.4(6) \\ \mathrm{C}(23)-\mathrm{C}(22)-\mathrm{C}(27) & 124.1(5) \\ \mathrm{C}(21)-\mathrm{C}(22)-\mathrm{Fe}(1) & 69.7(3) \\ \mathrm{C}(23)-\mathrm{C}(22)-\mathrm{Fe}(1) & 70.0(3) \\ \mathrm{C}(27)-\mathrm{C}(22)-\mathrm{Fe}(1) & 128.9(4) \\ \mathrm{C}(22)-\mathrm{C}(23)-\mathrm{C}(24) & 107.9(5) \\ \mathrm{C}(22)-\mathrm{C}(23)-\mathrm{C}(28) & 126.0(5) \\ \mathrm{C}(24)-\mathrm{C}(23)-\mathrm{C}(28) & 126.1(5) \\ \mathrm{C}(22)-\mathrm{C}(23)-\mathrm{Fe}(1) & 69.4(3) \\ \mathrm{C}(24)-\mathrm{C}(23)-\mathrm{Fe}(1) & 69.1(3) \\ \mathrm{C}(28)-\mathrm{C}(23)-\mathrm{Fe}(1) & 128.6(4) \\ \mathrm{C}(23)-\mathrm{C}(24)-\mathrm{C}(25) & 107.6(5) \\ \mathrm{C}(23)-\mathrm{C}(24)-\mathrm{C}(29) & 128.0(6) \\ \mathrm{C}(25)-\mathrm{C}(24)-\mathrm{C}(29) & 124.4(5) \\ \mathrm{C}(23)-\mathrm{C}(24)-\mathrm{Fe}(1) & 70.1(3) \\ \mathrm{C}(25)-\mathrm{C}(24)-\mathrm{Fe}(1) & 69.4(3) \\ \mathrm{C}(29)-\mathrm{C}(24)-\mathrm{Fe}(1) & 127.4(4) \\ \mathrm{C}(24)-\mathrm{C}(25)-\mathrm{C}(21) & 107.9(5) \\ \mathrm{C}(24)-\mathrm{C}(25)-\mathrm{C}(30) & 127.1(5) \\ \mathrm{C}(21)-\mathrm{C}(25)-\mathrm{C}(30) & 125.0(5) \\ \mathrm{C}(24)-\mathrm{C}(25)-\mathrm{Fe}(1) & 69.7(3) \\ \mathrm{C}(21)-\mathrm{C}(25)-\mathrm{Fe}(1) & 69.7(3) \\ \mathrm{C}(30)-\mathrm{C}(25)-\mathrm{Fe}(1) & 127.3(4) \\ \mathrm{C}(123)-\mathrm{Fe}(2)-\mathrm{C}(104) & 124.9(2) \\ \mathrm{C}(123)-\mathrm{Fe}(2)-\mathrm{C}(122) & 41.1(2) \\ \mathrm{C}(104)-\mathrm{Fe}(2)-\mathrm{C}(122) & 162.1(2) \\ \mathrm{C}(123)-\mathrm{Fe}(2)-\mathrm{C}(121) & 68.7(2) \\ & \end{array}$




\begin{tabular}{|c|c|}
\hline $\mathrm{C}(104)-\mathrm{Fe}(2)-\mathrm{C}(121)$ & $156.5(2)$ \\
\hline $\mathrm{C}(122)-\mathrm{Fe}(2)-\mathrm{C}(121)$ & $40.2(2)$ \\
\hline $\mathrm{C}(123)-\mathrm{Fe}(2)-\mathrm{C}(125)$ & $68.8(2)$ \\
\hline $\mathrm{C}(104)-\mathrm{Fe}(2)-\mathrm{C}(125)$ & $120.9(2)$ \\
\hline $\mathrm{C}(122)-\mathrm{Fe}(2)-\mathrm{C}(125)$ & $68.6(2)$ \\
\hline $\mathrm{C}(121)-\mathrm{Fe}(2)-\mathrm{C}(125)$ & $41.3(2)$ \\
\hline $\mathrm{C}(123)-\mathrm{Fe}(2)-\mathrm{C}(105)$ & $163.8(2)$ \\
\hline$C(104)-F e(2)-C(105)$ & $40.8(2)$ \\
\hline $\mathrm{C}(122)-\mathrm{Fe}(2)-\mathrm{C}(105)$ & $154.8(2)$ \\
\hline$C(121)-F e(2)-C(105)$ & $121.8(2)$ \\
\hline $\mathrm{C}(125)-\mathrm{Fe}(2)-\mathrm{C}(105)$ & $110.0(2)$ \\
\hline$C(123)-F e(2)-C(102)$ & $116.8(2)$ \\
\hline$C(104)-F e(2)-C(102)$ & $68.0(2)$ \\
\hline $\mathrm{C}(122)-\mathrm{Fe}(2)-\mathrm{C}(102)$ & $106.2(2)$ \\
\hline$C(121)-F e(2)-C(102)$ & $126.4(2)$ \\
\hline $\mathrm{C}(125)-\mathrm{Fe}(2)-\mathrm{C}(102)$ & $165.6(2)$ \\
\hline$C(105)-F e(2)-C(102)$ & $68.5(2)$ \\
\hline$C(123)-\mathrm{Fe}(2)-\mathrm{C}(124)$ & $40.6(2)$ \\
\hline $\mathrm{C}(104)-\mathrm{Fe}(2)-\mathrm{C}(124)$ & 108.1(2) \\
\hline $\mathrm{C}(122)-\mathrm{Fe}(2)-\mathrm{C}(124)$ & $68.4(2)$ \\
\hline $\mathrm{C}(121)-\mathrm{Fe}(2)-\mathrm{C}(124)$ & $68.5(2)$ \\
\hline $\mathrm{C}(125)-\mathrm{Fe}(2)-\mathrm{C}(124)$ & $40.6(2)$ \\
\hline $\mathrm{C}(105)-\mathrm{Fe}(2)-\mathrm{C}(124)$ & $128.1(2)$ \\
\hline $\mathrm{C}(102)-\mathrm{Fe}(2)-\mathrm{C}(124)$ & $151.5(2)$ \\
\hline$C(123)-F e(2)-C(103)$ & $105.7(2)$ \\
\hline $\mathrm{C}(104)-\mathrm{Fe}(2)-\mathrm{C}(103)$ & $40.1(2)$ \\
\hline$C(122)-\mathrm{Fe}(2)-\mathrm{C}(103)$ & $124.9(2)$ \\
\hline$C(121)-F e(2)-C(103)$ & $162.5(2)$ \\
\hline$C(125)-\mathrm{Fe}(2)-\mathrm{C}(103)$ & $153.9(2)$ \\
\hline$C(105)-F e(2)-C(103)$ & $67.9(2)$ \\
\hline$C(102)-F e(2)-C(103)$ & $40.0(2)$ \\
\hline$C(124)-F e(2)-C(103)$ & $118.7(2)$ \\
\hline$C(123)-F e(2)-C(101)$ & $151.4(2)$ \\
\hline$C(104)-F e(2)-C(101)$ & $68.0(2)$ \\
\hline$C(122)-F e(2)-C(101)$ & $119.1(2)$ \\
\hline $\mathrm{C}(121)-\mathrm{Fe}(2)-\mathrm{C}(101)$ & $110.0(2)$ \\
\hline
\end{tabular}




$\begin{array}{lr}\mathrm{C}(125)-\mathrm{Fe}(2)-\mathrm{C}(101) & 130.2(2) \\ \mathrm{C}(105)-\mathrm{Fe}(2)-\mathrm{C}(101) & 41.34(19) \\ \mathrm{C}(102)-\mathrm{Fe}(2)-\mathrm{C}(101) & 39.4(2) \\ \mathrm{C}(124)-\mathrm{Fe}(2)-\mathrm{C}(101) & 167.6(2) \\ \mathrm{C}(103)-\mathrm{Fe}(2)-\mathrm{C}(101) & 66.5(2) \\ \mathrm{O}(102)-\mathrm{S}(101)-\mathrm{O}(101) & 116.9(2) \\ \mathrm{O}(102)-\mathrm{S}(101)-\mathrm{N}(103) & 110.3(2) \\ \mathrm{O}(101)-\mathrm{S}(101)-\mathrm{N}(103) & 117.2(2) \\ \mathrm{O}(102)-\mathrm{S}(101)-\mathrm{C}(120) & 101.3(3) \\ \mathrm{O}(101)-\mathrm{S}(101)-\mathrm{C}(120) & 101.5(3) \\ \mathrm{N}(103)-\mathrm{S}(101)-\mathrm{C}(120) & 107.4(3) \\ \mathrm{C}(108)-\mathrm{N}(101)-\mathrm{C}(101) & 118.6(5) \\ \mathrm{C}(108)-\mathrm{N}(101)-\mathrm{C}(113) & 122.0(5) \\ \mathrm{C}(101)-\mathrm{N}(101)-\mathrm{C}(113) & 119.4(5) \\ \mathrm{C}(106)-\mathrm{N}(102)-\mathrm{C}(112) & 123.6(5) \\ \mathrm{C}(106)-\mathrm{N}(102)-\mathrm{C}(109) & 125.2(5) \\ \mathrm{C}(112)-\mathrm{N}(102)-\mathrm{C}(109) & 110.8(4) \\ \mathrm{C}(113)-\mathrm{N}(103)-\mathrm{S}(101) & 118.9(4) \\ \mathrm{N}(101)-\mathrm{C}(101)-\mathrm{C}(102) & 130.9(5) \\ \mathrm{N}(101)-\mathrm{C}(101)-\mathrm{C}(105) & 120.8(5) \\ \mathrm{C}(102)-\mathrm{C}(101)-\mathrm{C}(105) & 108.3(5) \\ \mathrm{N}(101)-\mathrm{C}(101)-\mathrm{Fe}(2) & 126.4(4) \\ \mathrm{C}(102)-\mathrm{C}(101)-\mathrm{Fe}(2) & 69.3(3) \\ \mathrm{C}(105)-\mathrm{C}(101)-\mathrm{Fe}(2) & 68.4(3) \\ \mathrm{C}(101)-\mathrm{C}(102)-\mathrm{C}(103) & 108.3(5) \\ \mathrm{C}(101)-\mathrm{C}(102)-\mathrm{Fe}(2) & 71.2(3) \\ \mathrm{C}(103)-\mathrm{C}(102)-\mathrm{Fe}(2) & 70.2(3) \\ \mathrm{C}(104)-\mathrm{C}(103)-\mathrm{C}(102) & 109.0(5) \\ \mathrm{C}(104)-\mathrm{C}(103)-\mathrm{Fe}(2) & 69.1(3) \\ \mathrm{C}(102)-\mathrm{C}(103)-\mathrm{Fe}(2) & 69.8(3) \\ \mathrm{C}(103)-\mathrm{C}(104)-\mathrm{C}(105) & 108.4(5) \\ \mathrm{C}(103)-\mathrm{C}(104)-\mathrm{Fe}(2) & 70.8(3) \\ \mathrm{C}(105)-\mathrm{C}(104)-\mathrm{Fe}(2) & 70.2(3) \\ \mathrm{C}(104)-\mathrm{C}(105)-\mathrm{C}(106) & 135.2(5) \\ \mathrm{C}(104)-\mathrm{C}(105)-\mathrm{C}(101) & 105.9(5) \\ \mathrm{C}(106)-\mathrm{C}(105)-\mathrm{C}(101) & 118.8(5)\end{array}$




$\begin{array}{lr}\mathrm{C}(104)-\mathrm{C}(105)-\mathrm{Fe}(2) & 69.0(3) \\ \mathrm{C}(106)-\mathrm{C}(105)-\mathrm{Fe}(2) & 121.7(4) \\ \mathrm{C}(101)-\mathrm{C}(105)-\mathrm{Fe}(2) & 70.3(3) \\ \mathrm{N}(102)-\mathrm{C}(106)-\mathrm{C}(107) & 120.1(5) \\ \mathrm{N}(102)-\mathrm{C}(106)-\mathrm{C}(105) & 124.0(5) \\ \mathrm{C}(107)-\mathrm{C}(106)-\mathrm{C}(105) & 116.0(5) \\ \mathrm{C}(108)-\mathrm{C}(107)-\mathrm{C}(106) & 122.1(6) \\ \mathrm{N}(101)-\mathrm{C}(108)-\mathrm{C}(107) & 123.6(5) \\ \mathrm{N}(102)-\mathrm{C}(109)-\mathrm{C}(110) & 102.8(5) \\ \mathrm{C}(111)-\mathrm{C}(110)-\mathrm{C}(109) & 103.6(5) \\ \mathrm{C}(110)-\mathrm{C}(111)-\mathrm{C}(112) & 103.3(5) \\ \mathrm{N}(102)-\mathrm{C}(112)-\mathrm{C}(111) & 103.3(5) \\ \mathrm{N}(103)-\mathrm{C}(113)-\mathrm{C}(114) & 111.7(4) \\ \mathrm{N}(103)-\mathrm{C}(113)-\mathrm{N}(101) & 107.7(4) \\ \mathrm{C}(114)-\mathrm{C}(113)-\mathrm{N}(101) & 109.7(4) \\ \mathrm{C}(119)-\mathrm{C}(114)-\mathrm{C}(115) & 118.9(6) \\ \mathrm{C}(119)-\mathrm{C}(114)-\mathrm{C}(113) & 121.2(5) \\ \mathrm{C}(115)-\mathrm{C}(114)-\mathrm{C}(113) & 119.9(5) \\ \mathrm{C}(116)-\mathrm{C}(115)-\mathrm{C}(114) & 120.2(6) \\ \mathrm{C}(117)-\mathrm{C}(116)-\mathrm{C}(115) & 119.7(6) \\ \mathrm{C}(118)-\mathrm{C}(117)-\mathrm{C}(116) & 120.3(6) \\ \mathrm{C}(117)-\mathrm{C}(118)-\mathrm{C}(119) & 120.5(6) \\ \mathrm{C}(114)-\mathrm{C}(119)-\mathrm{C}(118) & 120.3(6) \\ \mathrm{F}(102)-\mathrm{C}(120)-\mathrm{F}(101) & 107.3(5) \\ \mathrm{F}(102)-\mathrm{C}(120)-\mathrm{F}(103) & 107.4(5) \\ \mathrm{F}(101)-\mathrm{C}(120)-\mathrm{F}(103) & 107.4(5) \\ \mathrm{F}(102)-\mathrm{C}(120)-\mathrm{S}(101) & 111.6(4) \\ \mathrm{F}(101)-\mathrm{C}(120)-\mathrm{S}(101) & 112.1(4) \\ \mathrm{F}(103)-\mathrm{C}(120)-\mathrm{S}(101) & 110.7(4) \\ \mathrm{C}(122)-\mathrm{C}(121)-\mathrm{C}(125) & 108.1(5) \\ \mathrm{C}(122)-\mathrm{C}(121)-\mathrm{C}(126) & 127.9(5) \\ \mathrm{C}(125)-\mathrm{C}(121)-\mathrm{C}(126) & 123.9(5) \\ \mathrm{C}(122)-\mathrm{C}(121)-\mathrm{Fe}(2) & 69.8(3) \\ \mathrm{C}(125)-\mathrm{C}(121)-\mathrm{Fe}(2) & 69.5(3) \\ \mathrm{C}(126)-\mathrm{C}(121)-\mathrm{Fe}(2) & 128.5(4) \\ \mathrm{C}(121)-\mathrm{C}(122)-\mathrm{C}(123) & 108.5(5) \\ & \end{array}$




$\begin{array}{lr}\mathrm{C}(121)-\mathrm{C}(122)-\mathrm{C}(127) & 126.2(5) \\ \mathrm{C}(123)-\mathrm{C}(122)-\mathrm{C}(127) & 125.3(5) \\ \mathrm{C}(121)-\mathrm{C}(122)-\mathrm{Fe}(2) & 70.0(3) \\ \mathrm{C}(123)-\mathrm{C}(122)-\mathrm{Fe}(2) & 69.2(3) \\ \mathrm{C}(127)-\mathrm{C}(122)-\mathrm{Fe}(2) & 127.9(4) \\ \mathrm{C}(124)-\mathrm{C}(123)-\mathrm{C}(122) & 107.7(5) \\ \mathrm{C}(124)-\mathrm{C}(123)-\mathrm{C}(128) & 127.6(6) \\ \mathrm{C}(122)-\mathrm{C}(123)-\mathrm{C}(128) & 124.7(5) \\ \mathrm{C}(124)-\mathrm{C}(123)-\mathrm{Fe}(2) & 70.5(3) \\ \mathrm{C}(122)-\mathrm{C}(123)-\mathrm{Fe}(2) & 69.7(3) \\ \mathrm{C}(128)-\mathrm{C}(123)-\mathrm{Fe}(2) & 127.2(4) \\ \mathrm{C}(123)-\mathrm{C}(124)-\mathrm{C}(125) & 108.4(5) \\ \mathrm{C}(123)-\mathrm{C}(124)-\mathrm{C}(129) & 126.5(6) \\ \mathrm{C}(125)-\mathrm{C}(124)-\mathrm{C}(129) & 125.1(6) \\ \mathrm{C}(123)-\mathrm{C}(124)-\mathrm{Fe}(2) & 68.9(3) \\ \mathrm{C}(125)-\mathrm{C}(124)-\mathrm{Fe}(2) & 69.4(3) \\ \mathrm{C}(129)-\mathrm{C}(124)-\mathrm{Fe}(2) & 128.0(4) \\ \mathrm{C}(124)-\mathrm{C}(125)-\mathrm{C}(121) & 107.3(5) \\ \mathrm{C}(124)-\mathrm{C}(125)-\mathrm{C}(130) & 127.4(5) \\ \mathrm{C}(121)-\mathrm{C}(125)-\mathrm{C}(130) & 125.2(6) \\ \mathrm{C}(124)-\mathrm{C}(125)-\mathrm{Fe}(2) & 70.0(3) \\ \mathrm{C}(121)-\mathrm{C}(125)-\mathrm{Fe}(2) & 69.2(3) \\ \mathrm{C}(130)-\mathrm{C}(125)-\mathrm{Fe}(2) & 129.9(4) \\ \mathrm{N}(15)-\mathrm{C}(15)-\mathrm{C}(2 \mathrm{~S}) & 176.1(9) \\ \mathrm{N}(2 \mathrm{~S})-\mathrm{C}(3 \mathrm{~S})-\mathrm{C}(4 \mathrm{~S}) & 178.0(7) \\ \mathrm{N}(35)-\mathrm{C}(55)-\mathrm{C}(6 \mathrm{~S}) & 177.0(8) \\ & \end{array}$

Symmetry transformations used to generate equivalent atoms: 
Table 4. Anisotropic displacement parameters $\left(\AA^{2} \times 10^{3}\right)$ for A. The anisotropic displacement factor exponent takes the form: $-2_{-}^{2}\left[h^{2} a^{* 2} U^{11}+\ldots+2 h k a^{*} b^{*} U^{12}\right]$

\begin{tabular}{|c|c|c|c|c|c|}
\hline $\mathrm{U}^{11}$ & $\mathrm{U}^{22}$ & $\mathrm{U}^{33}$ & $U^{23}$ & $\mathrm{U}^{13}$ & $\mathrm{U}^{12}$ \\
\hline $\mathrm{Fe}(1) 16(1)$ & $22(1)$ & $22(1)$ & $-2(1)$ & $1(1)$ & $1(1)$ \\
\hline$S(1) \quad 24(1)$ & $27(1)$ & 29(1) & $-1(1)$ & $-1(1)$ & $-6(1)$ \\
\hline $\mathrm{F}(1) \quad 37(2)$ & $34(2)$ & $65(3)$ & $-1(2)$ & $-4(2)$ & $-17(2)$ \\
\hline$F(2) \quad 33(2)$ & $45(3)$ & $139(4)$ & $-10(3)$ & $41(2)$ & $-3(2)$ \\
\hline$F(3) \quad 60(3)$ & $51(3)$ & $41(2)$ & $9(2)$ & $3(2)$ & $-21(2)$ \\
\hline $\mathrm{O}(1) 58(3)$ & $44(3)$ & $34(3)$ & $3(2)$ & $-23(2)$ & $-21(3)$ \\
\hline $\mathrm{O}(2) 26(3)$ & $31(3)$ & $57(3)$ & $-20(2)$ & $12(2)$ & $-2(2)$ \\
\hline $\mathrm{N}(1) \quad 9(3)$ & 19(3) & $21(2)$ & $6(2)$ & $2(2)$ & $1(2)$ \\
\hline $\mathrm{N}(2) 19(3)$ & 19(3) & 19(3) & $2(2)$ & $1(2)$ & $-4(2)$ \\
\hline $\mathrm{N}(3)$ 23(3) & $24(3)$ & $26(3)$ & $4(2)$ & $-6(2)$ & $-1(2)$ \\
\hline$C(1) 19(4)$ & $24(4)$ & $17(3)$ & $-3(3)$ & $-2(3)$ & $2(3)$ \\
\hline$C(2) 27(4)$ & $20(4)$ & $16(3)$ & $3(3)$ & 2(3) & $-2(3)$ \\
\hline$C(3) 20(3)$ & $23(4)$ & $23(3)$ & $-1(3)$ & $-2(2)$ & $5(3)$ \\
\hline$C(4) \quad 10(3)$ & $21(4)$ & $24(3)$ & $0(3)$ & $3(3)$ & $1(3)$ \\
\hline$C(5) \quad 16(3)$ & $16(3)$ & 16(3) & $-3(3)$ & $3(2)$ & $-5(3)$ \\
\hline$C(6) \quad 15(3)$ & $18(3)$ & $15(3)$ & $-4(3)$ & $-1(2)$ & $4(3)$ \\
\hline$C(7) 20(3)$ & $25(4)$ & 19(3) & $8(3)$ & $5(3)$ & $5(3)$ \\
\hline$C(8) \quad 14(3)$ & $23(4)$ & $17(3)$ & 1(3) & $1(2)$ & $3(3)$ \\
\hline$C(9) 20(3)$ & $16(4)$ & $30(3)$ & $-3(3)$ & $-4(3)$ & $-4(3)$ \\
\hline$C(10) 23(4)$ & $27(4)$ & $31(3)$ & $7(3)$ & 1(3) & $-4(3)$ \\
\hline $\mathrm{C}(11) 32(4)$ & $27(4)$ & 18(3) & 2(3) & $3(3)$ & $-4(3)$ \\
\hline $\mathrm{C}(12) 29(4)$ & $23(4)$ & $27(3)$ & $7(3)$ & $-1(3)$ & $-3(3)$ \\
\hline$C(13) 18(3)$ & $23(3)$ & $24(3)$ & $0(3)$ & $-1(3)$ & $-1(3)$ \\
\hline$C(14) 15(3)$ & $22(3)$ & 20(3) & 6(3) & $0(2)$ & $-11(3)$ \\
\hline$C(15) 23(4)$ & $34(4)$ & $40(4)$ & $10(3)$ & $3(3)$ & $-7(3)$ \\
\hline$C(16) 31(4)$ & $62(5)$ & $31(4)$ & $26(4)$ & $-1(3)$ & $-19(4)$ \\
\hline $\mathrm{C}(17) 31(4)$ & $71(6)$ & $27(4)$ & $-2(4)$ & $11(3)$ & $-23(4)$ \\
\hline$C(18) 28(4)$ & $55(5)$ & $33(4)$ & $-9(4)$ & $7(3)$ & $-7(3)$ \\
\hline$C(19) 14(3)$ & $37(4)$ & $31(4)$ & $-7(3)$ & 1(3) & $-6(3)$ \\
\hline$C(20) 22(4)$ & $31(4)$ & $61(5)$ & $-10(4)$ & $3(4)$ & $7(4)$ \\
\hline $\mathrm{C}(21) 30(4)$ & $14(3)$ & $33(3)$ & $-7(3)$ & $10(3)$ & $3(3)$ \\
\hline
\end{tabular}




\begin{tabular}{|c|c|c|c|c|c|}
\hline $\mathrm{C}(22) 22(4)$ & $26(4)$ & $22(3)$ & $-18(3)$ & $9(3)$ & $-7(3)$ \\
\hline$C(23) 24(4)$ & $24(4)$ & 23(3) & $-4(3)$ & $5(3)$ & $4(3)$ \\
\hline$C(24) 24(4)$ & $27(4)$ & $22(3)$ & $0(3)$ & $4(3)$ & $-5(3)$ \\
\hline$C(25) 15(3)$ & $29(4)$ & $30(3)$ & $-2(3)$ & 2(3) & $3(3)$ \\
\hline $\mathrm{C}(26) 36(4)$ & $28(4)$ & $54(4)$ & $-13(4)$ & $3(3)$ & $-4(3)$ \\
\hline$C(27) 23(4)$ & $53(5)$ & $27(3)$ & $-17(3)$ & $-2(3)$ & $0(3)$ \\
\hline $\mathrm{C}(28) 31(4)$ & $39(4)$ & $24(3)$ & $-4(3)$ & 1(3) & $4(3)$ \\
\hline$C(29) 20(4)$ & $37(4)$ & $31(3)$ & $3(3)$ & $10(3)$ & $4(3)$ \\
\hline$C(30) 25(3)$ & $33(4)$ & $48(4)$ & $5(4)$ & $8(3)$ & $6(3)$ \\
\hline $\mathrm{Fe}(2) 23(1)$ & $24(1)$ & $21(1)$ & $-2(1)$ & $4(1)$ & $2(1)$ \\
\hline $\mathrm{S}(101) 30(1)$ & $28(1)$ & $24(1)$ & $-3(1)$ & $-3(1)$ & $-1(1)$ \\
\hline $\mathrm{F}(101) 28(2)$ & $63(3)$ & $53(2)$ & $-6(2)$ & $3(2)$ & $-2(2)$ \\
\hline $\mathrm{F}(102) 49(2)$ & $34(2)$ & $44(2)$ & $-6(2)$ & $-13(2)$ & $6(2)$ \\
\hline $\mathrm{F}(103) 45(2)$ & $54(3)$ & $36(2)$ & $-8(2)$ & $-17(2)$ & $1(2)$ \\
\hline $\mathrm{O}(101) 35(2)$ & $39(3)$ & $20(2)$ & $-3(2)$ & $5(2)$ & $-2(2)$ \\
\hline $\mathrm{O}(102) 42(3)$ & $24(3)$ & $29(2)$ & $0(2)$ & $-7(2)$ & $-3(2)$ \\
\hline $\mathrm{N}(101) 26(3)$ & $23(3)$ & $17(3)$ & $2(2)$ & $1(2)$ & $-1(3)$ \\
\hline $\mathrm{N}(102) 25(3)$ & $20(3)$ & $22(3)$ & $6(2)$ & $-1(2)$ & $1(2)$ \\
\hline $\mathrm{N}(103) 31(3)$ & $30(3)$ & $17(2)$ & $0(2)$ & $-3(2)$ & $-4(3)$ \\
\hline$C(101) 27(4)$ & $24(4)$ & $17(3)$ & $-7(3)$ & $8(3)$ & $-7(3)$ \\
\hline $\mathrm{C}(102) 20(4)$ & $29(4)$ & $14(3)$ & $7(3)$ & $0(3)$ & $4(3)$ \\
\hline$C(103) 25(4)$ & $17(4)$ & $27(3)$ & $1(3)$ & $10(3)$ & $-1(3)$ \\
\hline$C(104) 25(4)$ & $23(4)$ & 21(3) & $4(3)$ & $3(3)$ & $2(3)$ \\
\hline $\mathrm{C}(105) 20(3)$ & $20(4)$ & $16(3)$ & $0(3)$ & $4(2)$ & $7(3)$ \\
\hline$C(106) 20(3)$ & $28(4)$ & $16(3)$ & $-8(3)$ & $3(3)$ & $1(3)$ \\
\hline$C(107) 38(4)$ & $22(3)$ & $18(3)$ & $0(3)$ & $6(3)$ & $-3(4)$ \\
\hline C(108)27(4) & $27(4)$ & 21(3) & $-6(3)$ & $3(3)$ & $-4(3)$ \\
\hline C(109)29(4) & $24(4)$ & $33(4)$ & $0(3)$ & $0(3)$ & $2(3)$ \\
\hline $\mathrm{C}(110) 41(4)$ & $30(4)$ & $42(4)$ & $2(3)$ & $-7(3)$ & $5(3)$ \\
\hline$C(111) 45(4)$ & $37(4)$ & $30(4)$ & $3(3)$ & $-6(3)$ & $15(3)$ \\
\hline$C(112) 34(4)$ & $32(4)$ & $30(4)$ & $-1(3)$ & $-7(3)$ & $7(3)$ \\
\hline$C(113) 24(4)$ & $23(4)$ & 22(3) & $8(3)$ & $-1(3)$ & $-3(3)$ \\
\hline C(114)18(3) & $34(4)$ & $15(3)$ & $-2(3)$ & $-7(3)$ & $-1(3)$ \\
\hline$C(115) 24(4)$ & $35(4)$ & 22(3) & $1(3)$ & $-3(3)$ & $-6(3)$ \\
\hline C(116)18(4) & $54(5)$ & $26(4)$ & $-10(4)$ & $5(3)$ & $3(4)$ \\
\hline$C(117) 26(4)$ & $42(5)$ & $31(4)$ & $-19(3)$ & $-10(3)$ & $9(3)$ \\
\hline
\end{tabular}




\begin{tabular}{lllccc}
$\mathrm{C}(118) 26(4)$ & $35(4)$ & $32(4)$ & $-4(3)$ & $-11(3)$ & $6(3)$ \\
$\mathrm{C}(119) 19(4)$ & $31(4)$ & $23(3)$ & $0(3)$ & $-3(3)$ & $3(3)$ \\
$\mathrm{C}(120) 37(4)$ & $35(4)$ & $28(4)$ & $-9(3)$ & $0(3)$ & $0(4)$ \\
$\mathrm{C}(121) 31(4)$ & $25(4)$ & $19(3)$ & $-3(3)$ & $6(3)$ & $3(3)$ \\
$\mathrm{C}(122) 30(4)$ & $20(4)$ & $21(3)$ & $-6(3)$ & $11(3)$ & $7(3)$ \\
$\mathrm{C}(123) 41(4)$ & $24(4)$ & $24(3)$ & $1(3)$ & $16(3)$ & $4(3)$ \\
$\mathrm{C}(124) 27(4)$ & $33(5)$ & $35(4)$ & $-6(3)$ & $15(3)$ & $2(3)$ \\
$\mathrm{C}(125) 29(4)$ & $28(4)$ & $26(3)$ & $-4(3)$ & $10(3)$ & $10(3)$ \\
$\mathrm{C}(126) 45(4)$ & $25(4)$ & $24(3)$ & $-5(3)$ & $4(3)$ & $4(3)$ \\
$\mathrm{C}(127) 41(4)$ & $40(4)$ & $25(3)$ & $-4(3)$ & $2(3)$ & $7(4)$ \\
$\mathrm{C}(128) 57(5)$ & $42(5)$ & $45(4)$ & $4(4)$ & $26(4)$ & $-11(4)$ \\
$\mathrm{C}(129) 26(4)$ & $55(5)$ & $58(4)$ & $-23(4)$ & $14(3)$ & $-1(4)$ \\
$\mathrm{C}(130) 33(4)$ & $41(5)$ & $34(4)$ & $-9(3)$ & $4(3)$ & $15(3)$ \\
$\mathrm{N}(1 \mathrm{~S}) 97(6)$ & $50(5)$ & $70(5)$ & $20(4)$ & $-20(4)$ & $-21(4)$ \\
$\mathrm{C}(1 S) 64(6)$ & $37(5)$ & $61(5)$ & $0(4)$ & $-10(5)$ & $-8(4)$ \\
$\mathrm{C}(2 S) 63(6)$ & $71(6)$ & $59(5)$ & $-9(5)$ & $5(4)$ & $-4(5)$ \\
$\mathrm{N}(2 S) 43(4)$ & $56(5)$ & $57(4)$ & $-3(4)$ & $22(3)$ & $-6(3)$ \\
$\mathrm{C}(3 S) 22(4)$ & $39(5)$ & $45(5)$ & $11(4)$ & $10(3)$ & $2(3)$ \\
$\mathrm{C}(4 S) 33(4)$ & $45(5)$ & $40(4)$ & $11(4)$ & $4(3)$ & $0(3)$ \\
$\mathrm{N}(3 S) 77(5)$ & $33(4)$ & $55(4)$ & $-3(3)$ & $-11(4)$ & $11(4)$ \\
$\mathrm{C}(5 S) 85(7)$ & $28(4)$ & $36(5)$ & $1(4)$ & $-2(4)$ & $20(4)$ \\
$\mathrm{C}(6 S) 82(6)$ & $56(5)$ & $39(4)$ & $-9(4)$ & $-27(4)$ & $34(5)$ \\
& & & & & \\
\hline
\end{tabular}


Table 5. Hydrogen coordinates ( $\left.x 10^{4}\right)$ and isotropic displacement parameters $\left(\AA^{2} \mathrm{x}\right.$ $10^{3}$ ) for $\mathbf{A}$.

\begin{tabular}{lrrrl}
\hline & $\mathrm{x}$ & $\mathrm{y}$ & $\mathrm{z}$ & $\mathrm{U}(\mathrm{eq})$ \\
\hline & & & \\
$\mathrm{H}(2)$ & -2245 & -8872 & -2451 & 26 \\
$\mathrm{H}(3)$ & -490 & -8134 & -2044 & 27 \\
$\mathrm{H}(4)$ & -285 & -6484 & -2651 & 22 \\
$\mathrm{H}(7)$ & -3430 & -5552 & -4322 & 25 \\
$\mathrm{H}(8)$ & -4448 & -6880 & -4089 & 22 \\
$\mathrm{H}(9 \mathrm{~A})$ & -59 & -5628 & -3592 & 27 \\
$\mathrm{H}(9 \mathrm{~B})$ & -389 & -4765 & -3055 & 27 \\
$\mathrm{H}(10 \mathrm{~A})$ & 153 & -4504 & -4488 & 32 \\
$\mathrm{H}(10 \mathrm{~B})$ & 477 & -3794 & -3814 & 32 \\
$\mathrm{H}(11 \mathrm{~A})$ & -1009 & -3181 & -4669 & 31 \\
$\mathrm{H}(11 \mathrm{~B})$ & -1173 & -3098 & -3831 & 31 \\
$\mathrm{H}(12 \mathrm{~A})$ & -2587 & -4071 & -4188 & 32 \\
$\mathrm{H}(12 \mathrm{~B})$ & -1997 & -4611 & -4805 & 32 \\
$\mathrm{H}(13)$ & -3766 & -9098 & -3295 & 26 \\
$\mathrm{H}(15)$ & -3764 & -9687 & -2139 & 39 \\
$\mathrm{H}(16)$ & -4286 & -9628 & -987 & 50 \\
$\mathrm{H}(17)$ & -5382 & -8351 & -653 & 51 \\
$\mathrm{H}(18)$ & -5968 & -7144 & -1487 & 47 \\
$\mathrm{H}(19)$ & -5397 & -7152 & -2627 & 33 \\
$\mathrm{H}(26 \mathrm{~A})$ & -2397 & -10248 & -3931 & 59 \\
$\mathrm{H}(26 \mathrm{~B})$ & -1504 & -10502 & -3320 & 59 \\
$\mathrm{H}(26 \mathrm{C})$ & -1397 & -10870 & -4119 & 59 \\
$\mathrm{H}(27 \mathrm{~A})$ & -2394 & -8912 & -5380 & 52 \\
$\mathrm{H}(27 \mathrm{~B})$ & -2717 & -7897 & -5001 & 52 \\
$\mathrm{H}(27 \mathrm{C})$ & -3015 & -8961 & -4681 & 52 \\
$\mathrm{H}(28 \mathrm{~A})$ & -217 & -6442 & -4772 & 47 \\
$\mathrm{H}(28 \mathrm{~B})$ & -1434 & -6617 & -4943 & 47 \\
$\mathrm{H}(28 \mathrm{C})$ & -629 & -7114 & -5439 & 47 \\
$\mathrm{H}(29 \mathrm{~A})$ & 1727 & -7920 & -4107 & 43 \\
$\mathrm{H}(29 \mathrm{~B})$ & 1397 & -7628 & -3333 & 43 \\
& & & &
\end{tabular}




\begin{tabular}{|c|c|c|c|}
\hline $\mathrm{H}(29 \mathrm{C})$ & 1117 & -6895 & -3993 \\
\hline $\mathrm{H}(30 \mathrm{~A})$ & 1000 & -10165 & -3438 \\
\hline $\mathrm{H}(30 \mathrm{~B})$ & 150 & -10073 & -2870 \\
\hline $\mathrm{H}(30 \mathrm{C})$ & 1050 & -9252 & -2885 \\
\hline $\mathrm{H}(102)$ & -1550 & -5945 & -895 \\
\hline $\mathrm{H}(103)$ & -3195 & -6760 & -1415 \\
\hline $\mathrm{H}(104)$ & -4054 & -5615 & -2346 \\
\hline $\mathrm{H}(107)$ & -1987 & -2446 & -2708 \\
\hline $\mathrm{H}(108)$ & -584 & -2794 & -1935 \\
\hline $\mathrm{H}(10 \mathrm{C})$ & -4868 & -4263 & -2627 \\
\hline $\mathrm{H}(10 \mathrm{D})$ & -4575 & -4641 & -3398 \\
\hline $\mathrm{H}(11 \mathrm{C})$ & -5950 & -3616 & -3750 \\
\hline $\mathrm{H}(11 \mathrm{D})$ & -5708 & -2861 & -3089 \\
\hline $\mathrm{H}(11 \mathrm{E})$ & -5045 & -2003 & -4014 \\
\hline $\mathrm{H}(11 \mathrm{~F})$ & -4579 & -3014 & -4335 \\
\hline $\mathrm{H}(11 \mathrm{G})$ & -3122 & -2433 & -3687 \\
\hline $\mathrm{H}(11 \mathrm{H})$ & -3783 & -1944 & -3085 \\
\hline $\mathrm{H}(113)$ & -320 & -4572 & -614 \\
\hline $\mathrm{H}(115)$ & 1072 & -4171 & -2182 \\
\hline $\mathrm{H}(116)$ & 2182 & -5339 & -2652 \\
\hline $\mathrm{H}(117)$ & 2296 & -6966 & -2186 \\
\hline $\mathrm{H}(118)$ & 1406 & -7391 & -1211 \\
\hline $\mathrm{H}(119)$ & 300 & -6236 & -731 \\
\hline $\mathrm{H}(12 \mathrm{~A})$ & -2712 & -2139 & -105 \\
\hline $\mathrm{H}(12 \mathrm{~B})$ & -2431 & -2351 & -903 \\
\hline $\mathrm{H}(12 \mathrm{C})$ & -1781 & -2883 & -249 \\
\hline $\mathrm{H}(12 \mathrm{D})$ & -1689 & -4312 & 418 \\
\hline $\mathrm{H}(12 \mathrm{E})$ & -2183 & -5408 & 472 \\
\hline $\mathrm{H}(12 \mathrm{~F})$ & -2551 & -4518 & 964 \\
\hline $\mathrm{H}(12 \mathrm{G})$ & -4602 & -5904 & 486 \\
\hline $\mathrm{H}(12 \mathrm{H})$ & -3649 & -6391 & 118 \\
\hline $\mathrm{H}(12 \mathrm{I})$ & -4778 & -6373 & -296 \\
\hline $\mathrm{H}(12 \mathrm{~J})$ & -5729 & -5503 & -1228 \\
\hline $\mathrm{H}(12 \mathrm{~K})$ & -5837 & -4441 & -1623 \\
\hline $\mathrm{H}(12 \mathrm{~L})$ & -6270 & -4582 & -858 \\
\hline $\mathrm{H}(13 \mathrm{~A})$ & -5028 & -2969 & -1805 \\
\hline
\end{tabular}




\begin{tabular}{lrrrl}
$\mathrm{H}(13 \mathrm{~B})$ & -3957 & -2385 & -1635 & 54 \\
$\mathrm{H}(13 \mathrm{C})$ & -4900 & -2201 & -1152 & 54 \\
$\mathrm{H}(2 \mathrm{~S} 1)$ & 2029 & -12092 & -1395 & 96 \\
$\mathrm{H}(2 \mathrm{~S} 2)$ & 2669 & -11345 & -1860 & 96 \\
$\mathrm{H}(2 \mathrm{~S} 3)$ & 2917 & -12522 & -1855 & 96 \\
$\mathrm{H}(4 \mathrm{~S} 1)$ & -6168 & -6311 & -5031 & 59 \\
$\mathrm{H}(4 \mathrm{~S} 2)$ & -7333 & -6623 & -4905 & 59 \\
$\mathrm{H}(4 \mathrm{~S} 3)$ & -6391 & -7344 & -4638 & 59 \\
$\mathrm{H}(6 \mathrm{~S} 1)$ & 299 & -10483 & -1618 & 91 \\
$\mathrm{H}(6 \mathrm{~S} 2)$ & -85 & -11000 & -920 & 91 \\
$\mathrm{H}(6 \mathrm{~S} 3)$ & -193 & -9813 & -1027 & 91 \\
& & & & \\
\hline
\end{tabular}




\section{Determination of Relative and Absolute Configuration}

Structure 1. A clear, colorless diethyl ether solution of the amide produced in eq 5 was prepared. Crystals suitable for X-ray structural analysis were obtained by diffusing pentane into the ether solution at room temperature.

A colorless needle of dimensions $0.4 \times 0.19 \times 0.16 \mathrm{~mm}^{3}$ was mounted under STP and transferred to a Bruker AXS/CCD three-circle diffractometer equipped with a cold stream of $\mathrm{N}_{2}$ gas. An initial unit cell was determined with monochromated $\mathrm{Mo} \mathrm{K \alpha}$ radiation $(\lambda=0.71073 \AA)$. The cell thus determined was triclinic.

The raw data frames were integrated using the Bruker program SAINT+ for NT version 6.01. The data that were collected (4966 total reflections, 4073 unique, $R_{\text {int }}=$ 0.0266) had the following Miller index ranges: -9 to 8 in $h,-12$ to 13 in $k$, and -13 to 10 in 1. SADABS absorption correction was performed, and the maximum and minimum effective transmissions were 0.808038 and 0.251115 , respectively.

All aspects of the solution and refinement were handled by SHELXTL NT version 5.10. The structure was solved by direct methods in the triclinic space group $\mathrm{P} 1, \mathrm{a}=$ 8.7897(7) $\AA ; b=12.2246(9) \AA ; c=12.4432(9) \AA ; \alpha=93.8300(10)^{\circ} ; \beta=108.5630(10)^{\circ} ; \gamma=$ $100.5330(10)^{\circ}$, and refined using standard difference Fourier techniques. Final, fullmatrix least-squares refinement (4073 data for 616 parameters) on F2 yielded residuals

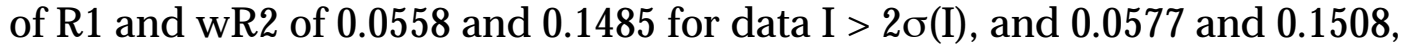
respectively, for all data. During the final refinement, all non-hydrogen atoms were treated anisotropically. Hydrogen atoms were included in calculated positions and refined isotropically on a riding model. No extinction coefficient was used in the refinement. Residual electron density amounted to a maximum of $0.871 \mathrm{e} / \AA^{3}$ and a minimum of $-0.562 \mathrm{e} / \AA^{3}$.

The absolute structure (Flack) parameter for the correct enantiomer is 0.060(12). The structure was inverted and refined in order to confirm the initial assignment of absolute stereochemistry.

Tables 1-6 provide the full crystallographic data for the X-ray structure. 


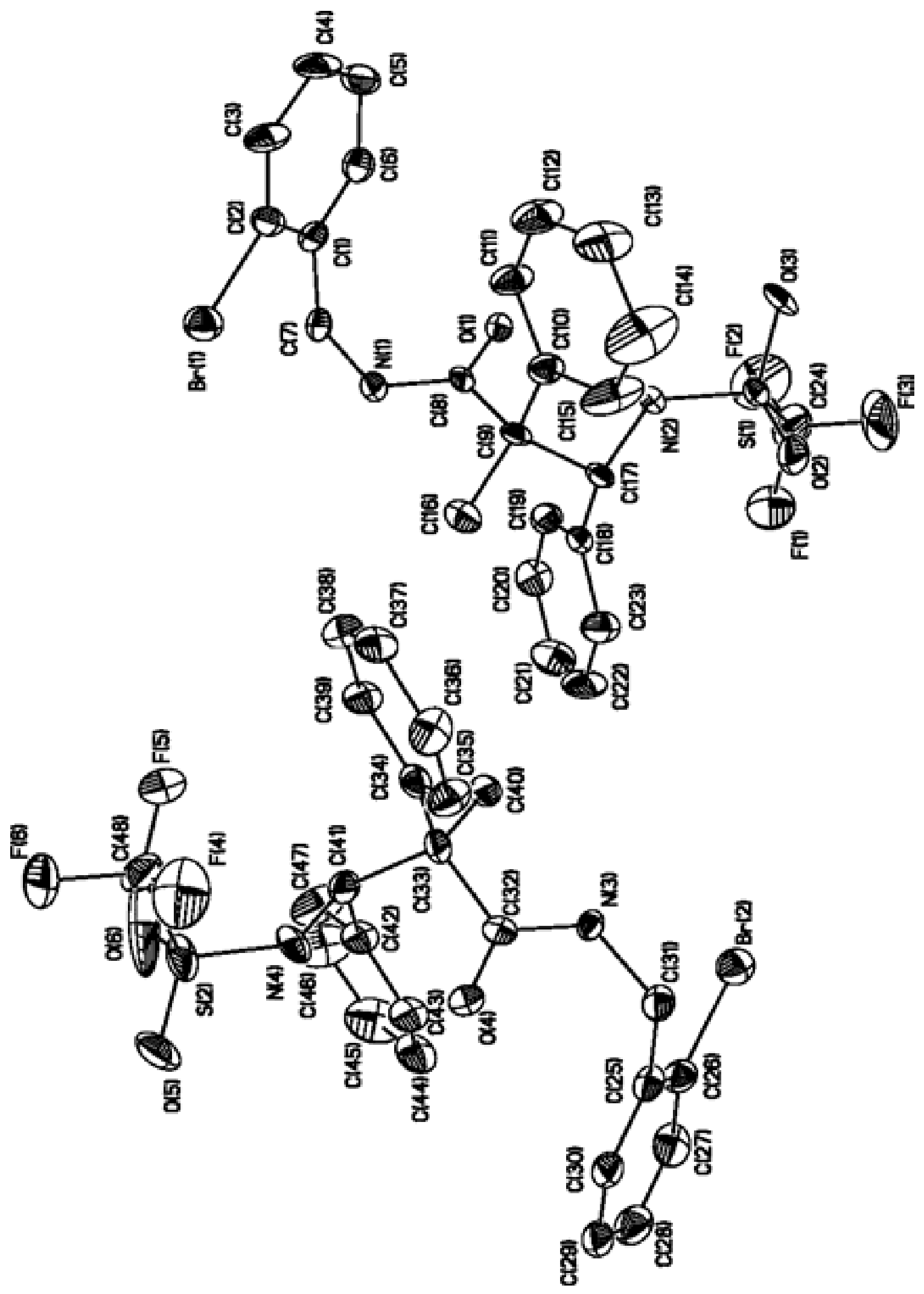




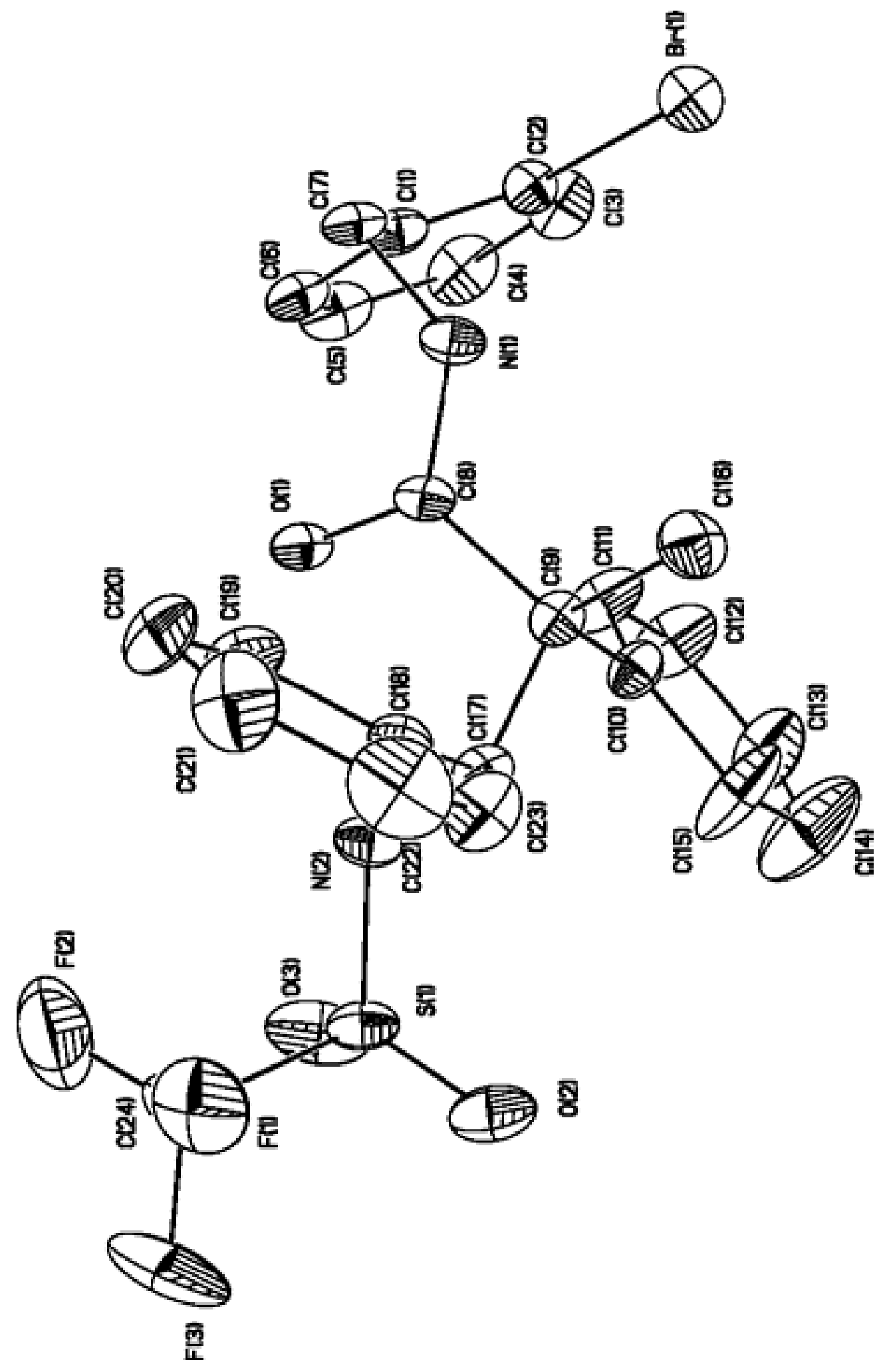




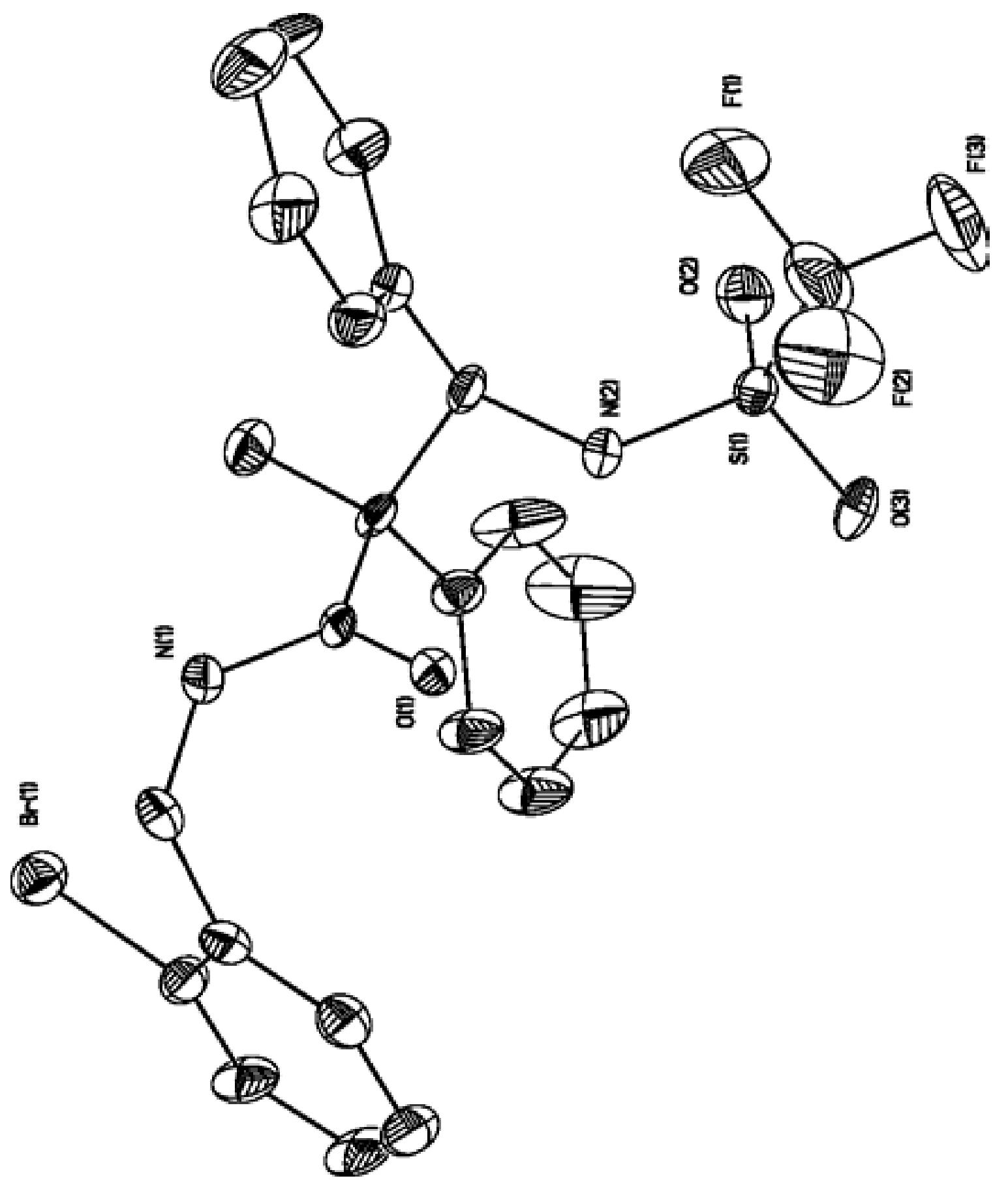


Table 1. Crystal data and structure refinement for 02117CSs.

Identification code

Empirical formula

Formula weight

Temperature

Wavelength

Crystal system

Space group

Unit cell dimensions

Volume

Z

Density (calculated)

Absorption coefficient

$\mathrm{F}(000)$

Crystal size

Theta range for data collection

Index ranges

Reflections collected

Independent reflections

Completeness to theta $=23.26^{\circ}$

Refinement method

Data / restraints / parameters

Goodness-of-fit on $\mathrm{F}^{2}$

Final $\mathrm{R}$ indices [I $>2$ sigma(I)]

$\mathrm{R}$ indices (all data)

Absolute structure parameter

Extinction coefficient

Largest diff. peak and hole
$02117 \mathrm{css}$

C24 H22 Br F3 N2 O3 S

555.41

183(2) K

$0.71073 \AA$

Triclinic

P1

$\mathrm{a}=8.7897(7) \AA \quad \mathrm{a}=93.8300(10)^{\circ}$.

$\mathrm{b}=12.2246(9) \AA \quad \mathrm{b}=108.5630(10)^{\circ}$.

$\mathrm{c}=12.4432(9) \AA \quad \mathrm{g}=100.5330(10)^{\circ}$.

1234.85(16) $\AA^{3}$

2

$1.494 \mathrm{Mg} / \mathrm{m}^{3}$

$1.800 \mathrm{~mm}^{-1}$

564

$0.4 \times 0.19 \times 0.16 \mathrm{~mm}^{3}$

2.50 to $23.26^{\circ}$.

$-9<=\mathrm{h}<=8,-12<=\mathrm{k}<=13,-13<=\mathrm{l}<=10$

4966

$4073[\mathrm{R}(\mathrm{int})=0.0266]$

$98.1 \%$

Full-matrix least-squares on $\mathrm{F}^{2}$

4073 / 3 / 616

1.025

$\mathrm{R} 1=0.0558, \mathrm{wR} 2=0.1485$

$\mathrm{R} 1=0.0577, \mathrm{wR} 2=0.1508$

$0.060(12)$

$0.000(2)$

0.871 and -0.562 e. $\AA^{-3}$ 
Table 2. Atomic coordinates ( $\left.\times 10^{4}\right)$ and equivalent isotropic displacement parameters $\left(\AA^{2} \times 10^{3}\right)$ for $02117 C S s$. U(eq) is defined as one third of the trace of the orthogonalized Uij tensor.

\begin{tabular}{|c|c|c|c|c|}
\hline & $x$ & $\mathrm{y}$ & z & $\mathrm{U}(\mathrm{eq})$ \\
\hline $\operatorname{Br}(1)$ & 2073(1) & $1478(1)$ & 1915(1) & $50(1)$ \\
\hline $\operatorname{Br}(2)$ & $11635(1)$ & $6258(1)$ & $11354(1)$ & $54(1)$ \\
\hline$S(1)$ & 12078(3) & $1857(2)$ & $5741(2)$ & $40(1)$ \\
\hline$S(2)$ & 1852(3) & 6546(3) & 7544(2) & $59(1)$ \\
\hline $\mathrm{F}(1)$ & $12571(10)$ & $1407(7)$ & $7820(6)$ & $84(2)$ \\
\hline$F(2)$ & $12443(14)$ & $30(8)$ & 6638(9) & $124(4)$ \\
\hline$F(3)$ & $14608(10)$ & $1349(10)$ & 7197(9) & $124(4)$ \\
\hline$F(4)$ & $2138(12)$ & 7641(9) & $5871(8)$ & $107(3)$ \\
\hline$F(5)$ & $1627(10)$ & $5906(10)$ & $5467(8)$ & $121(4)$ \\
\hline$F(6)$ & $-301(9)$ & 6628(9) & 5613(7) & 103(3) \\
\hline $\mathrm{O}(1)$ & 7305(7) & $174(5)$ & 4195(5) & $37(2)$ \\
\hline $\mathrm{O}(2)$ & 12658(9) & 2983(7) & $6210(7)$ & $59(2)$ \\
\hline $\mathrm{O}(3)$ & $12505(9)$ & 1382(9) & 4815(7) & $77(3)$ \\
\hline $\mathrm{O}(4)$ & $6736(8)$ & 7843(5) & $8856(5)$ & $41(2)$ \\
\hline $\mathrm{O}(5)$ & $1540(12)$ & $7575(11)$ & 7980(8) & $108(4)$ \\
\hline $\mathrm{O}(6)$ & 1037(11) & $5499(14)$ & $7578(14)$ & $160(7)$ \\
\hline $\mathrm{N}(1)$ & $4976(9)$ & 769(7) & 4021(7) & $37(2)$ \\
\hline $\mathrm{N}(2)$ & 10159(9) & $1475(7)$ & $5449(7)$ & $38(2)$ \\
\hline $\mathrm{N}(3)$ & $8883(9)$ & $7044(6)$ & $9126(6)$ & $33(2)$ \\
\hline $\mathrm{N}(4)$ & $3754(10)$ & 6624(7) & 7947(7) & $42(2)$ \\
\hline$C(1)$ & $3870(11)$ & $-284(8)$ & 2113(9) & $41(2)$ \\
\hline$C(2)$ & $3153(12)$ & $426(8)$ & 1378(9) & $38(2)$ \\
\hline$C(3)$ & $3210(15)$ & $459(9)$ & $306(9)$ & $53(3)$ \\
\hline$C(4)$ & $4067(16)$ & $-229(10)$ & $-81(10)$ & $58(3)$ \\
\hline$C(5)$ & $4767(15)$ & $-962(11)$ & $576(11)$ & $58(3)$ \\
\hline$C(6)$ & $4682(12)$ & $-984(9)$ & $1693(10)$ & $47(3)$ \\
\hline$C(7)$ & 3913(11) & $-281(8)$ & 3345(9) & $40(2)$ \\
\hline$C(8)$ & $6611(10)$ & 952(7) & $4327(7)$ & $29(2)$ \\
\hline$C(9)$ & $7612(10)$ & $2160(6)$ & 4781(7) & $30(2)$ \\
\hline$C(10)$ & $8127(12)$ & $2630(7)$ & $3810(8)$ & $40(2)$ \\
\hline
\end{tabular}




\begin{tabular}{|c|c|c|c|c|}
\hline $\mathrm{C}(11)$ & $7522(14)$ & 2085(9) & 2707(9) & $56(3)$ \\
\hline$C(12)$ & 7943(18) & $2546(11)$ & $1823(10)$ & $71(4)$ \\
\hline$C(13)$ & $9030(20)$ & $3521(11)$ & 2033(11) & $72(4)$ \\
\hline$C(14)$ & $9690(30)$ & $4106(15)$ & $3123(16)$ & $123(8)$ \\
\hline$C(15)$ & 9250(20) & $3626(10)$ & 4011(11) & $84(5)$ \\
\hline$C(16)$ & 6649(13) & 2943(9) & 5199(10) & $50(3)$ \\
\hline $\mathrm{C}(17)$ & $9166(10)$ & 2173(7) & $5845(8)$ & $33(2)$ \\
\hline$C(18)$ & $8831(10)$ & 1791(7) & 6891(7) & $33(2)$ \\
\hline $\mathrm{C}(19)$ & $8230(12)$ & $710(8)$ & 6996(8) & $46(2)$ \\
\hline$C(20)$ & $8004(14)$ & 433(9) & $8002(10)$ & $52(3)$ \\
\hline$C(21)$ & $8388(17)$ & $1222(11)$ & 8914(11) & $65(3)$ \\
\hline$C(22)$ & 8953(19) & $2278(14)$ & $8838(11)$ & $69(4)$ \\
\hline$C(23)$ & $9223(14)$ & 2611(9) & $7842(10)$ & $52(3)$ \\
\hline $\mathrm{C}(24)$ & 12939(16) & 1086(13) & 6910(13) & $80(4)$ \\
\hline$C(25)$ & $9917(11)$ & $8060(8)$ & 11087(8) & $39(2)$ \\
\hline$C(26)$ & 10554(13) & 7342(9) & 11828(9) & $46(3)$ \\
\hline$C(27)$ & 10468(15) & $7340(11)$ & $12935(10)$ & $60(3)$ \\
\hline$C(28)$ & $9737(16)$ & 8103(11) & 13295(11) & $61(3)$ \\
\hline$C(29)$ & 9032(13) & 8824(11) & $12567(10)$ & $55(3)$ \\
\hline$C(30)$ & $9171(12)$ & $8821(10)$ & $11500(9)$ & $48(3)$ \\
\hline$C(31)$ & $10027(12)$ & $8033(9)$ & $9910(8)$ & $41(2)$ \\
\hline$C(32)$ & 7303(11) & 7033(7) & $8666(7)$ & $32(2)$ \\
\hline$C(33)$ & $6226(10)$ & $5958(7)$ & $7856(8)$ & $38(2)$ \\
\hline$C(34)$ & $5873(11)$ & $6238(8)$ & $6599(7)$ & $39(2)$ \\
\hline$C(35)$ & $6462(13)$ & 7230(8) & $6326(9)$ & $51(2)$ \\
\hline$C(36)$ & $6187(13)$ & $7443(10)$ & 5198(9) & $54(3)$ \\
\hline$C(37)$ & $5295(14)$ & $6591(10)$ & $4324(9)$ & $50(3)$ \\
\hline$C(38)$ & $4675(14)$ & $5554(10)$ & 4591(10) & $54(3)$ \\
\hline$C(39)$ & $4954(13)$ & $5373(8)$ & 5702(9) & $44(2)$ \\
\hline$C(40)$ & 7053(11) & $4950(8)$ & $8035(9)$ & $38(2)$ \\
\hline $\mathrm{C}(41)$ & $4557(11)$ & $5672(8)$ & $8104(9)$ & $42(2)$ \\
\hline$C(42)$ & $4762(12)$ & $5252(8)$ & $9269(8)$ & $44(2)$ \\
\hline$C(43)$ & $5651(13)$ & 5902(9) & 10291(9) & $51(3)$ \\
\hline $\mathrm{C}(44)$ & $5745(16)$ & $5499(12)$ & $11299(11)$ & $62(3)$ \\
\hline$C(45)$ & $4920(30)$ & $4458(14)$ & 11332(15) & $94(5)$ \\
\hline$C(46)$ & $3930(20)$ & $3811(12)$ & $10254(14)$ & $91(5)$ \\
\hline
\end{tabular}


C(47)

C(48)
3930(20)

1333(16)
4207(11)

9279(12)

6004(11)
79(4)

66(4) 
Table 3. Bond lengths $[\AA]$ and angles $\left[{ }^{\circ}\right]$ for $02117 C S s$.

\begin{tabular}{ll}
\hline $\mathrm{Br}(1)-\mathrm{C}(2)$ & $1.933(9)$ \\
$\mathrm{Br}(2)-\mathrm{C}(26)$ & $1.929(11)$ \\
$\mathrm{S}(1)-\mathrm{O}(2)$ & $1.393(8)$ \\
$\mathrm{S}(1)-\mathrm{O}(3)$ & $1.440(7)$ \\
$\mathrm{S}(1)-\mathrm{N}(2)$ & $1.580(8)$ \\
$\mathrm{S}(1)-\mathrm{C}(24)$ & $1.823(15)$ \\
$\mathrm{S}(2)-\mathrm{O}(6)$ & $1.357(13)$ \\
$\mathrm{S}(2)-\mathrm{O}(5)$ & $1.444(10)$ \\
$\mathrm{S}(2)-\mathrm{N}(4)$ & $1.567(8)$ \\
$\mathrm{S}(2)-\mathrm{C}(48)$ & $1.855(14)$ \\
$\mathrm{F}(1)-\mathrm{C}(24)$ & $1.328(17)$ \\
$\mathrm{F}(2)-\mathrm{C}(24)$ & $1.270(16)$ \\
$\mathrm{F}(3)-\mathrm{C}(24)$ & $1.364(15)$ \\
$\mathrm{F}(4)-\mathrm{C}(48)$ & $1.280(16)$ \\
$\mathrm{F}(5)-\mathrm{C}(48)$ & $1.260(13)$ \\
$\mathrm{F}(6)-\mathrm{C}(48)$ & $1.344(14)$ \\
$\mathrm{O}(1)-\mathrm{C}(8)$ & $1.249(10)$ \\
$\mathrm{O}(4)-\mathrm{C}(32)$ & $1.229(11)$ \\
$\mathrm{N}(1)-\mathrm{C}(8)$ & $1.335(11)$ \\
$\mathrm{N}(1)-\mathrm{C}(7)$ & $1.465(12)$ \\
$\mathrm{N}(2)-\mathrm{C}(17)$ & $1.490(10)$ \\
$\mathrm{N}(3)-\mathrm{C}(32)$ & $1.320(12)$ \\
$\mathrm{N}(3)-\mathrm{C}(31)$ & $1.482(12)$ \\
$\mathrm{N}(4)-\mathrm{C}(41)$ & $1.460(13)$ \\
$\mathrm{C}(1)-\mathrm{C}(6)$ & $1.384(15)$ \\
$\mathrm{C}(1)-\mathrm{C}(2)$ & $1.388(15)$ \\
$\mathrm{C}(1)-\mathrm{C}(7)$ & $1.521(15)$ \\
$\mathrm{C}(2)-\mathrm{C}(3)$ & $1.353(14)$ \\
$\mathrm{C}(3)-\mathrm{C}(4)$ & $1.385(17)$ \\
$\mathrm{C}(4)-\mathrm{C}(5)$ & $1.351(19)$ \\
$\mathrm{C}(5)-\mathrm{C}(6)$ & $1.418(17)$ \\
$\mathrm{C}(8)-\mathrm{C}(9)$ & $1.540(11)$ \\
$\mathrm{C}(9)-\mathrm{C}(10)$ & $1.531(13)$ \\
$\mathrm{C}(9)-\mathrm{C}(16)$ & $1.553(11)$ \\
& \\
& \\
& \\
& \\
& \\
& \\
& \\
&
\end{tabular}




$\begin{array}{ll}\mathrm{C}(9)-\mathrm{C}(17) & 1.567(12) \\ \mathrm{C}(10)-\mathrm{C}(15) & 1.373(14) \\ \mathrm{C}(10)-\mathrm{C}(11) & 1.376(14) \\ \mathrm{C}(11)-\mathrm{C}(12) & 1.390(16) \\ \mathrm{C}(12)-\mathrm{C}(13) & 1.337(19) \\ \mathrm{C}(13)-\mathrm{C}(14) & 1.38(2) \\ \mathrm{C}(14)-\mathrm{C}(15) & 1.41(2) \\ \mathrm{C}(17)-\mathrm{C}(18) & 1.509(12) \\ \mathrm{C}(18)-\mathrm{C}(19) & 1.362(13) \\ \mathrm{C}(18)-\mathrm{C}(23) & 1.406(13) \\ \mathrm{C}(19)-\mathrm{C}(20) & 1.383(14) \\ \mathrm{C}(20)-\mathrm{C}(21) & 1.350(16) \\ \mathrm{C}(21)-\mathrm{C}(22) & 1.318(19) \\ \mathrm{C}(22)-\mathrm{C}(23) & 1.406(18) \\ \mathrm{C}(25)-\mathrm{C}(26) & 1.376(15) \\ \mathrm{C}(25)-\mathrm{C}(30) & 1.391(15) \\ \mathrm{C}(25)-\mathrm{C}(31) & 1.497(13) \\ \mathrm{C}(26)-\mathrm{C}(27) & 1.403(16) \\ \mathrm{C}(27)-\mathrm{C}(28) & 1.353(18) \\ \mathrm{C}(28)-\mathrm{C}(29) & 1.39(2) \\ \mathrm{C}(29)-\mathrm{C}(30) & 1.372(16) \\ \mathrm{C}(32)-\mathrm{C}(33) & 1.549(13) \\ \mathrm{C}(33)-\mathrm{C}(40) & 1.535(13) \\ \mathrm{C}(33)-\mathrm{C}(34) & 1.568(13) \\ \mathrm{C}(33)-\mathrm{C}(41) & 1.576(12) \\ \mathrm{C}(34)-\mathrm{C}(35) & 1.339(14) \\ \mathrm{C}(34)-\mathrm{C}(39) & 1.404(14) \\ \mathrm{C}(35)-\mathrm{C}(36) & 1.397(15) \\ \mathrm{C}(36)-\mathrm{C}(37) & 1.374(16) \\ \mathrm{C}(37)-\mathrm{C}(38) & 1.389(17) \\ \mathrm{C}(38)-\mathrm{C}(39) & 1.367(16) \\ \mathrm{C}(41)-\mathrm{C}(42) & 1.539(14) \\ \mathrm{C}(42)-\mathrm{C}(47) & 1.354(15) \\ \mathrm{C}(42)-\mathrm{C}(43) & 1.366(15) \\ \mathrm{C}(43)-\mathrm{C}(44) & 1.362(16) \\ \mathrm{C}(44)-\mathrm{C}(45) & 1.36(2)\end{array}$




$\begin{array}{ll}\mathrm{C}(45)-\mathrm{C}(46) & 1.43(2) \\ \mathrm{C}(46)-\mathrm{C}(47) & 1.34(2) \\ \mathrm{O}(2)-\mathrm{S}(1)-\mathrm{O}(3) & 122.5(5) \\ \mathrm{O}(2)-\mathrm{S}(1)-\mathrm{N}(2) & 112.0(4) \\ \mathrm{O}(3)-\mathrm{S}(1)-\mathrm{N}(2) & 108.4(4) \\ \mathrm{O}(2)-\mathrm{S}(1)-\mathrm{C}(24) & 104.5(6) \\ \mathrm{O}(3)-\mathrm{S}(1)-\mathrm{C}(24) & 103.5(6) \\ \mathrm{N}(2)-\mathrm{S}(1)-\mathrm{C}(24) & 104.0(6) \\ \mathrm{O}(6)-\mathrm{S}(2)-\mathrm{O}(5) & 124.8(9) \\ \mathrm{O}(6)-\mathrm{S}(2)-\mathrm{N}(4) & 111.0(6) \\ \mathrm{O}(5)-\mathrm{S}(2)-\mathrm{N}(4) & 109.0(5) \\ \mathrm{O}(6)-\mathrm{S}(2)-\mathrm{C}(48) & 105.7(9) \\ \mathrm{O}(5)-\mathrm{S}(2)-\mathrm{C}(48) & 100.5(6) \\ \mathrm{N}(4)-\mathrm{S}(2)-\mathrm{C}(48) & 103.1(5) \\ \mathrm{C}(8)-\mathrm{N}(1)-\mathrm{C}(7) & 121.8(8) \\ \mathrm{C}(17)-\mathrm{N}(2)-\mathrm{S}(1) & 123.0(6) \\ \mathrm{C}(32)-\mathrm{N}(3)-\mathrm{C}(31) & 120.7(8) \\ \mathrm{C}(41)-\mathrm{N}(4)-\mathrm{S}(2) & 125.6(7) \\ \mathrm{C}(6)-\mathrm{C}(1)-\mathrm{C}(2) & 116.1(10) \\ \mathrm{C}(6)-\mathrm{C}(1)-\mathrm{C}(7) & 119.2(10) \\ \mathrm{C}(2)-\mathrm{C}(1)-\mathrm{C}(7) & 124.6(8) \\ \mathrm{C}(3)-\mathrm{C}(2)-\mathrm{C}(1) & 123.9(9) \\ \mathrm{C}(3)-\mathrm{C}(2)-\mathrm{B}(1) & 117.1(8) \\ \mathrm{C}(1)-\mathrm{C}(2)-\mathrm{B}(1) & 119.0(7) \\ \mathrm{C}(2)-\mathrm{C}(3)-\mathrm{C}(4) & 118.8(11) \\ \mathrm{C}(5)-\mathrm{C}(4)-\mathrm{C}(3) & 120.8(10) \\ \mathrm{C}(4)-\mathrm{C}(5)-\mathrm{C}(6) & 119.3(11) \\ \mathrm{C}(1)-\mathrm{C}(6)-\mathrm{C}(5) & 121.1(11) \\ \mathrm{N}(1)-\mathrm{C}(7)-\mathrm{C}(1) & 108.9(8) \\ \mathrm{O}(1)-\mathrm{C}(8)-\mathrm{N}(1) & 120.8(8) \\ \mathrm{O}(1)-\mathrm{C}(8)-\mathrm{C}(9) & 120.8(7) \\ \mathrm{N}(1)-\mathrm{C}(8)-\mathrm{C}(9) & 118.3(7) \\ \mathrm{C}(10)-\mathrm{C}(9)-\mathrm{C}(8) & 107.4(6) \\ \mathrm{C}(10)-\mathrm{C}(9)-\mathrm{C}(16) & 109.5(7) \\ \mathrm{C}(8)-\mathrm{C}(9)-\mathrm{C}(16) & 113.6(7) \\ \mathrm{C}(10)-\mathrm{C}(9)-\mathrm{C}(17) & 110.3(7) \\ & \end{array}$




$\begin{array}{ll}\mathrm{C}(8)-\mathrm{C}(9)-\mathrm{C}(17) & 110.7(6) \\ \mathrm{C}(16)-\mathrm{C}(9)-\mathrm{C}(17) & 105.4(7) \\ \mathrm{C}(15)-\mathrm{C}(10)-\mathrm{C}(11) & 116.6(10) \\ \mathrm{C}(15)-\mathrm{C}(10)-\mathrm{C}(9) & 120.7(8) \\ \mathrm{C}(11)-\mathrm{C}(10)-\mathrm{C}(9) & 122.7(8) \\ \mathrm{C}(10)-\mathrm{C}(11)-\mathrm{C}(12) & 122.0(10) \\ \mathrm{C}(13)-\mathrm{C}(12)-\mathrm{C}(11) & 120.5(11) \\ \mathrm{C}(12)-\mathrm{C}(13)-\mathrm{C}(14) & 120.1(12) \\ \mathrm{C}(13)-\mathrm{C}(14)-\mathrm{C}(15) & 118.8(14) \\ \mathrm{C}(10)-\mathrm{C}(15)-\mathrm{C}(14) & 121.8(12) \\ \mathrm{N}(2)-\mathrm{C}(17)-\mathrm{C}(18) & 111.4(7) \\ \mathrm{N}(2)-\mathrm{C}(17)-\mathrm{C}(9) & 106.4(6) \\ \mathrm{C}(18)-\mathrm{C}(17)-\mathrm{C}(9) & 115.8(6) \\ \mathrm{C}(19)-\mathrm{C}(18)-\mathrm{C}(23) & 117.8(9) \\ \mathrm{C}(19)-\mathrm{C}(18)-\mathrm{C}(17) & 124.9(7) \\ \mathrm{C}(23)-\mathrm{C}(18)-\mathrm{C}(17) & 117.3(8) \\ \mathrm{C}(18)-\mathrm{C}(19)-\mathrm{C}(20) & 120.9(9) \\ \mathrm{C}(21)-\mathrm{C}(20)-\mathrm{C}(19) & 121.1(10) \\ \mathrm{C}(22)-\mathrm{C}(21)-\mathrm{C}(20) & 119.6(11) \\ \mathrm{C}(21)-\mathrm{C}(22)-\mathrm{C}(23) & 121.9(12) \\ \mathrm{C}(22)-\mathrm{C}(23)-\mathrm{C}(18) & 118.7(11) \\ \mathrm{F}(2)-\mathrm{C}(24)-\mathrm{F}(1) & 109.6(15) \\ \mathrm{F}(2)-\mathrm{C}(24)-\mathrm{F}(3) & 108.8(11) \\ \mathrm{F}(1)-\mathrm{C}(24)-\mathrm{F}(3) & 107.9(11) \\ \mathrm{F}(2)-\mathrm{C}(24)-\mathrm{S}(1) & 112.5(10) \\ \mathrm{F}(1)-\mathrm{C}(24)-\mathrm{S}(1) & 110.1(8) \\ \mathrm{F}(3)-\mathrm{C}(24)-\mathrm{S}(1) & 107.8(12) \\ \mathrm{C}(26)-\mathrm{C}(25)-\mathrm{C}(30) & 115.9(9) \\ \mathrm{C}(26)-\mathrm{C}(25)-\mathrm{C}(31) & 122.3(9) \\ \mathrm{C}(30)-\mathrm{C}(25)-\mathrm{C}(31) & 121.8(10) \\ \mathrm{C}(25)-\mathrm{C}(26)-\mathrm{C}(27) & 123.9(10) \\ \mathrm{C}(25)-\mathrm{C}(26)-\mathrm{Br}(2) & 119.7(7) \\ \mathrm{C}(27)-\mathrm{C}(26)-\mathrm{B}(2) & 116.5(9) \\ \mathrm{C}(28)-\mathrm{C}(27)-\mathrm{C}(26) & 117.7(12) \\ \mathrm{C}(27)-\mathrm{C}(28)-\mathrm{C}(29) & 120.6(12) \\ \mathrm{C}(30)-\mathrm{C}(29)-\mathrm{C}(28) & 120.1(11) \\ & \end{array}$




$\begin{array}{ll}\mathrm{C}(29)-\mathrm{C}(30)-\mathrm{C}(25) & 121.7(11) \\ \mathrm{N}(3)-\mathrm{C}(31)-\mathrm{C}(25) & 111.9(8) \\ \mathrm{O}(4)-\mathrm{C}(32)-\mathrm{N}(3) & 121.4(8) \\ \mathrm{O}(4)-\mathrm{C}(32)-\mathrm{C}(33) & 122.3(8) \\ \mathrm{N}(3)-\mathrm{C}(32)-\mathrm{C}(33) & 116.3(8) \\ \mathrm{C}(40)-\mathrm{C}(33)-\mathrm{C}(32) & 112.3(7) \\ \mathrm{C}(40)-\mathrm{C}(33)-\mathrm{C}(34) & 110.9(7) \\ \mathrm{C}(32)-\mathrm{C}(33)-\mathrm{C}(34) & 107.2(7) \\ \mathrm{C}(40)-\mathrm{C}(33)-\mathrm{C}(41) & 109.0(8) \\ \mathrm{C}(32)-\mathrm{C}(33)-\mathrm{C}(41) & 107.9(7) \\ \mathrm{C}(34)-\mathrm{C}(33)-\mathrm{C}(41) & 109.3(7) \\ \mathrm{C}(35)-\mathrm{C}(34)-\mathrm{C}(39) & 117.9(9) \\ \mathrm{C}(35)-\mathrm{C}(34)-\mathrm{C}(33) & 124.2(8) \\ \mathrm{C}(39)-\mathrm{C}(34)-\mathrm{C}(33) & 117.8(8) \\ \mathrm{C}(34)-\mathrm{C}(35)-\mathrm{C}(36) & 123.1(9) \\ \mathrm{C}(37)-\mathrm{C}(36)-\mathrm{C}(35) & 118.8(10) \\ \mathrm{C}(36)-\mathrm{C}(37)-\mathrm{C}(38) & 119.0(10) \\ \mathrm{C}(39)-\mathrm{C}(38)-\mathrm{C}(37) & 120.9(10) \\ \mathrm{C}(38)-\mathrm{C}(39)-\mathrm{C}(34) & 120.3(10) \\ \mathrm{N}(4)-\mathrm{C}(41)-\mathrm{C}(42) & 113.5(8) \\ \mathrm{N}(4)-\mathrm{C}(41)-\mathrm{C}(33) & 110.0(8) \\ \mathrm{C}(42)-\mathrm{C}(41)-\mathrm{C}(33) & 112.8(8) \\ \mathrm{C}(47)-\mathrm{C}(42)-\mathrm{C}(43) & 118.5(10) \\ \mathrm{C}(47)-\mathrm{C}(42)-\mathrm{C}(41) & 118.0(10) \\ \mathrm{C}(43)-\mathrm{C}(42)-\mathrm{C}(41) & 123.4(9) \\ \mathrm{C}(44)-\mathrm{C}(43)-\mathrm{C}(42) & 120.9(11) \\ \mathrm{C}(45)-\mathrm{C}(44)-\mathrm{C}(43) & 121.7(14) \\ \mathrm{C}(44)-\mathrm{C}(45)-\mathrm{C}(46) & 116.6(14) \\ \mathrm{C}(47)-\mathrm{C}(46)-\mathrm{C}(45) & 120.3(14) \\ \mathrm{C}(46)-\mathrm{C}(47)-\mathrm{C}(42) & 121.9(13) \\ \mathrm{F}(5)-\mathrm{C}(48)-\mathrm{F}(4) & 109.8(14) \\ \mathrm{F}(5)-\mathrm{C}(48)-\mathrm{F}(6) & 107.4(9) \\ \mathrm{F}(4)-\mathrm{C}(48)-\mathrm{F}(6) & 112.7(11) \\ \mathrm{F}(5)-\mathrm{C}(48)-\mathrm{S}(2) & 110.6(10) \\ \mathrm{F}(4)-\mathrm{C}(48)-\mathrm{S}(2) & 110.9(8) \\ \mathrm{F}(6)-\mathrm{C}(48)-\mathrm{S}(2) & 105.4(10) \\ & \end{array}$


Table 4. Anisotropic displacement parameters $\left(\AA^{2} \times 10^{3}\right)$ for 02117CSs. The anisotropic displacement factor exponent takes the form: $-2 p^{2}\left[h^{2} a^{* 2} U^{11}+\ldots+2 h k a^{*}\right.$ $b^{*} \mathrm{U}^{12}$ ]

\begin{tabular}{|c|c|c|c|c|c|c|}
\hline & $\mathrm{U}^{11}$ & $U^{22}$ & $\mathrm{U}^{33}$ & $\mathrm{U}^{23}$ & $\mathrm{U}^{13}$ & $\mathrm{U}^{12}$ \\
\hline $\operatorname{Br}(1)$ & ) $55(1)$ & $56(1)$ & $43(1)$ & $7(1)$ & $19(1)$ & $15(1)$ \\
\hline $\operatorname{Br}(2)$ & $57(1)$ & $56(1)$ & $49(1)$ & $5(1)$ & $17(1)$ & $11(1)$ \\
\hline$S(1)$ & $27(1)$ & $59(2)$ & $33(1)$ & $0(1)$ & $17(1)$ & $0(1)$ \\
\hline$S(2)$ & $33(1)$ & $113(2)$ & $42(2)$ & $23(2)$ & $22(1)$ & $20(1)$ \\
\hline $\mathrm{F}(1)$ & $98(6)$ & $116(6)$ & $41(4)$ & $32(4)$ & $23(4)$ & $23(5)$ \\
\hline$F(2)$ & $153(9)$ & $86(6)$ & $123(8)$ & $23(5)$ & $14(7)$ & $58(6)$ \\
\hline$F(3)$ & $42(4)$ & $208(11)$ & $119(8)$ & $33(7)$ & $6(4)$ & $53(5)$ \\
\hline $\mathrm{F}(4)$ & $96(6)$ & $134(8)$ & $90(6)$ & $53(6)$ & $27(5)$ & $17(6)$ \\
\hline$F(5)$ & $75(5)$ & 182(10) & $78(6)$ & $-72(7)$ & $2(4)$ & $35(5)$ \\
\hline$F(6)$ & $50(4)$ & $166(8)$ & $77(5)$ & $-8(5)$ & $-9(4)$ & $48(5)$ \\
\hline $\mathrm{O}(1)$ & $28(3)$ & $41(3)$ & $39(4)$ & $-7(3)$ & $11(3)$ & 2(3) \\
\hline $\mathrm{O}(2)$ & $40(4)$ & $76(5)$ & $54(4)$ & $3(4)$ & $20(3)$ & $-9(3)$ \\
\hline $\mathrm{O}(3)$ & $44(4)$ & $140(8)$ & $58(5)$ & $-13(5)$ & $36(4)$ & $21(4)$ \\
\hline $\mathrm{O}(4)$ & $41(3)$ & $47(4)$ & $31(3)$ & $-4(3)$ & $9(3)$ & $7(3)$ \\
\hline $\mathrm{O}(5)$ & $75(6)$ & 196(11) & $60(6)$ & $-32(6)$ & $18(5)$ & $77(7)$ \\
\hline $\mathrm{O}(6)$ & $32(5)$ & $237(15)$ & $217(15)$ & 183(13) & $27(7)$ & $15(7)$ \\
\hline $\mathrm{N}(1)$ & $27(4)$ & $53(5)$ & $32(4)$ & $3(4)$ & $13(3)$ & $6(3)$ \\
\hline $\mathrm{N}(2)$ & $27(4)$ & $53(5)$ & $36(4)$ & $-2(3)$ & $15(3)$ & $10(3)$ \\
\hline $\mathrm{N}(3)$ & $24(4)$ & $48(5)$ & $22(4)$ & $-1(3)$ & $9(3)$ & $-5(3)$ \\
\hline $\mathrm{N}(4)$ & $39(5)$ & $64(5)$ & $42(5)$ & $20(4)$ & $31(4)$ & $19(4)$ \\
\hline$C(1)$ & $32(5)$ & $42(5)$ & $38(5)$ & $-9(4)$ & $9(4)$ & $-6(4)$ \\
\hline$C(2)$ & $41(5)$ & $39(5)$ & $37(6)$ & $1(4)$ & $18(5)$ & $8(4)$ \\
\hline$C(3)$ & $74(8)$ & $59(7)$ & $23(5)$ & $5(5)$ & $18(5)$ & $7(5)$ \\
\hline$C(4)$ & $83(9)$ & $68(7)$ & $29(6)$ & $-1(5)$ & $34(6)$ & $1(6)$ \\
\hline$C(5)$ & $57(7)$ & $60(7)$ & $55(8)$ & $-17(6)$ & $30(6)$ & $-2(5)$ \\
\hline$C(6)$ & $38(5)$ & $44(5)$ & $51(7)$ & $-3(5)$ & $16(5)$ & $-5(4)$ \\
\hline$C(7)$ & $25(4)$ & $44(5)$ & $44(6)$ & $-2(4)$ & $10(4)$ & $-4(4)$ \\
\hline$C(8)$ & $24(4)$ & $49(5)$ & 19(4) & $9(4)$ & $15(3)$ & $5(4)$ \\
\hline$C(9)$ & $36(5)$ & $33(4)$ & $29(4)$ & 1(3) & $22(4)$ & $6(3)$ \\
\hline$C(10$ & $47(5)$ & $31(4)$ & $40(5)$ & $-3(4)$ & $17(4)$ & $2(4)$ \\
\hline
\end{tabular}




\begin{tabular}{|c|c|c|c|c|c|}
\hline$C(11) 66(7)$ & $60(6)$ & $34(6)$ & $-4(5)$ & $26(5)$ & $-18(5)$ \\
\hline $\mathrm{C}(12) 92(9)$ & $79(8)$ & $34(6)$ & $1(6)$ & $33(6)$ & $-19(7)$ \\
\hline $\mathrm{C}(13) 115(11)$ & $63(8)$ & $48(8)$ & $21(6)$ & $47(8)$ & $-2(7)$ \\
\hline $\mathrm{C}(14) 180(20)$ & $86(11)$ & 73(11) & 19(9) & $42(11)$ & $-50(12)$ \\
\hline $\mathrm{C}(15) 142(13)$ & $44(7)$ & $48(7)$ & $-5(5)$ & $44(8)$ & $-40(7)$ \\
\hline$C(16) 49(6)$ & $53(6)$ & $56(7)$ & $-2(5)$ & $25(5)$ & $17(5)$ \\
\hline $\mathrm{C}(17) 31(4)$ & $35(4)$ & $39(5)$ & $-4(4)$ & $23(4)$ & $4(3)$ \\
\hline $\mathrm{C}(18) 27(4)$ & $43(5)$ & $30(5)$ & $2(4)$ & $13(4)$ & $9(4)$ \\
\hline $\mathrm{C}(19) 43(5)$ & $54(6)$ & $39(6)$ & $0(4)$ & $20(4)$ & $-3(4)$ \\
\hline $\mathrm{C}(20) 62(7)$ & $49(6)$ & $50(6)$ & $21(5)$ & $33(5)$ & $-3(5)$ \\
\hline$C(21) 89(10)$ & $76(9)$ & $48(8)$ & $23(6)$ & $41(7)$ & $24(7)$ \\
\hline $\mathrm{C}(22) 88(9)$ & $100(11)$ & $26(6)$ & $-1(6)$ & $26(6)$ & $27(8)$ \\
\hline$C(23) 63(7)$ & $46(6)$ & $47(6)$ & $0(5)$ & $27(5)$ & $4(5)$ \\
\hline $\mathrm{C}(24) 56(8)$ & $88(10)$ & $81(10)$ & $1(8)$ & $-6(7)$ & $37(7)$ \\
\hline$C(25) 34(5)$ & $57(6)$ & $18(5)$ & $7(4)$ & $6(4)$ & $-2(4)$ \\
\hline$C(26) 45(6)$ & $57(6)$ & $32(6)$ & $-2(5)$ & $14(5)$ & $0(5)$ \\
\hline $\mathrm{C}(27) 54(7)$ & $83(9)$ & $44(7)$ & $16(6)$ & $23(6)$ & $1(6)$ \\
\hline $\mathrm{C}(28) 56(7)$ & $73(8)$ & $45(7)$ & $-2(6)$ & $17(6)$ & $-3(6)$ \\
\hline$C(29) 34(5)$ & $80(8)$ & $44(7)$ & $-13(6)$ & $16(5)$ & $0(5)$ \\
\hline$C(30) 34(5)$ & $68(7)$ & $33(6)$ & $-6(5)$ & $5(4)$ & $6(5)$ \\
\hline$C(31) 34(5)$ & $63(6)$ & $24(5)$ & $6(4)$ & $8(4)$ & $5(4)$ \\
\hline$C(32) 40(5)$ & $47(5)$ & $16(4)$ & $7(3)$ & $19(4)$ & $5(4)$ \\
\hline$C(33) 24(4)$ & $48(5)$ & $42(5)$ & $4(4)$ & $16(4)$ & $5(4)$ \\
\hline$C(34) 33(5)$ & $63(6)$ & $27(5)$ & $9(4)$ & $12(4)$ & $19(4)$ \\
\hline$C(35) 58(6)$ & $53(6)$ & $36(5)$ & $2(4)$ & $13(5)$ & $1(5)$ \\
\hline$C(36) 54(6)$ & $63(7)$ & $41(6)$ & $20(5)$ & $18(5)$ & $-4(5)$ \\
\hline$C(37) 56(7)$ & $74(7)$ & $28(5)$ & $14(5)$ & $24(5)$ & $16(5)$ \\
\hline$C(38) 51(6)$ & $69(7)$ & $36(7)$ & $-8(5)$ & $11(5)$ & $7(5)$ \\
\hline $\mathrm{C}(39) 51(6)$ & $45(5)$ & $39(6)$ & $4(4)$ & $29(5)$ & $-3(4)$ \\
\hline$C(40) 37(5)$ & $41(5)$ & $40(6)$ & $3(4)$ & $19(4)$ & $4(4)$ \\
\hline$C(41) 33(5)$ & $46(5)$ & $42(6)$ & $-4(4)$ & $16(4)$ & $-7(4)$ \\
\hline$C(42) 52(6)$ & $49(5)$ & $37(5)$ & $8(4)$ & $30(5)$ & $-1(4)$ \\
\hline$C(43) 57(6)$ & $59(6)$ & $44(6)$ & $3(5)$ & $39(5)$ & $-7(5)$ \\
\hline$C(44) 66(7)$ & $95(9)$ & $43(7)$ & $20(6)$ & $35(6)$ & $25(6)$ \\
\hline$C(45) 145(16)$ & 89(11) & $80(11)$ & $36(9)$ & $72(11)$ & $31(10)$ \\
\hline$C(46) 146(15)$ & $57(8)$ & $75(10)$ & $16(7)$ & $55(10)$ & $-3(8)$ \\
\hline
\end{tabular}




$\begin{array}{lllccc}C(47) 113(11) & 58(8) & 51(7) & 2(6) & 30(7) & -20(7) \\ C(48) 61(7) & 60(7) & 53(7) & -18(6) & -18(6) & 29(6)\end{array}$


Table 5. Hydrogen coordinates $\left(x 10^{4}\right)$ and isotropic displacement parameters $\left(\AA^{2} x\right.$ $10^{3}$ ) for 02117 CSs.

\begin{tabular}{|c|c|c|c|c|}
\hline & $x$ & $\mathrm{y}$ & $\mathrm{z}$ & $\mathrm{U}(\mathrm{eq})$ \\
\hline $\mathrm{H}(3)$ & 2672 & 946 & -171 & 63 \\
\hline $\mathrm{H}(4)$ & 4165 & -187 & -816 & 70 \\
\hline $\mathrm{H}(5)$ & 5310 & -1456 & 292 & 69 \\
\hline $\mathrm{H}(6)$ & 5191 & -1488 & 2162 & 56 \\
\hline $\mathrm{H}(7 \mathrm{~A})$ & 2789 & -346 & 3376 & 48 \\
\hline $\mathrm{H}(7 \mathrm{~B})$ & 4342 & -929 & 3661 & 48 \\
\hline $\mathrm{H}(11)$ & 6793 & 1370 & 2545 & 67 \\
\hline $\mathrm{H}(12)$ & 7454 & 2166 & 1064 & 85 \\
\hline $\mathrm{H}(13)$ & 9358 & 3811 & 1432 & 87 \\
\hline $\mathrm{H}(14)$ & 10412 & 4819 & 3271 & 148 \\
\hline $\mathrm{H}(15)$ & 9758 & 4001 & 4771 & 100 \\
\hline $\mathrm{H}(16 \mathrm{~A})$ & 7388 & 3670 & 5555 & 75 \\
\hline $\mathrm{H}(16 \mathrm{~B})$ & 6213 & 2599 & 5759 & 75 \\
\hline $\mathrm{H}(16 \mathrm{C})$ & 5739 & 3054 & 4545 & 75 \\
\hline $\mathrm{H}(17)$ & 9825 & 2961 & 6062 & 40 \\
\hline $\mathrm{H}(19)$ & 7962 & 139 & 6369 & 55 \\
\hline $\mathrm{H}(20)$ & 7572 & -326 & 8053 & 62 \\
\hline $\mathrm{H}(21)$ & 8250 & 1016 & 9606 & 78 \\
\hline $\mathrm{H}(22)$ & 9186 & 2832 & 9475 & 83 \\
\hline $\mathrm{H}(23)$ & 9661 & 3374 & 7812 & 62 \\
\hline $\mathrm{H}(27)$ & 10909 & 6821 & 13415 & 72 \\
\hline $\mathrm{H}(28)$ & 9705 & 8146 & 14052 & 73 \\
\hline $\mathrm{H}(29)$ & 8453 & 9320 & 12809 & 65 \\
\hline $\mathrm{H}(30)$ & 8746 & 9353 & 11031 & 57 \\
\hline $\mathrm{H}(31 \mathrm{~A})$ & 9766 & 8727 & 9599 & 50 \\
\hline $\mathrm{H}(31 \mathrm{~B})$ & 11166 & 8015 & 9953 & 50 \\
\hline $\mathrm{H}(35)$ & 7095 & 7818 & 6928 & 61 \\
\hline $\mathrm{H}(36)$ & 6609 & 8162 & 5038 & 64 \\
\hline $\mathrm{H}(37)$ & 5105 & 6709 & 3550 & 60 \\
\hline $\mathrm{H}(38)$ & 4049 & 4963 & 3991 & 65 \\
\hline
\end{tabular}




\begin{tabular}{lrrrr}
$\mathrm{H}(39)$ & 4525 & 4658 & 5869 & 52 \\
$\mathrm{H}(40 \mathrm{~A})$ & 7963 & 5055 & 7731 & 57 \\
$\mathrm{H}(40 \mathrm{~B})$ & 6248 & 4261 & 7633 & 57 \\
$\mathrm{H}(40 \mathrm{C})$ & 7476 & 4891 & 8854 & 57 \\
$\mathrm{H}(41)$ & 3824 & 5040 & 7506 & 50 \\
$\mathrm{H}(43)$ & 6212 & 6646 & 10299 & 61 \\
$\mathrm{H}(44)$ & 6408 & 5958 & 11998 & 75 \\
$\mathrm{H}(45)$ & 4989 & 4173 & 12034 & 113 \\
$\mathrm{H}(46)$ & 3264 & 3096 & 10230 & 110 \\
$\mathrm{H}(47)$ & 3330 & 3742 & 8573 & 94 \\
& & & & \\
\hline
\end{tabular}


Table 6. Torsion angles $\left[^{\circ}\right]$ for 02117 CSs.

\begin{tabular}{lc}
\hline $\mathrm{O}(2)-\mathrm{S}(1)-\mathrm{N}(2)-\mathrm{C}(17)$ & $-11.3(9)$ \\
$\mathrm{O}(3)-\mathrm{S}(1)-\mathrm{N}(2)-\mathrm{C}(17)$ & $-149.3(8)$ \\
$\mathrm{C}(24)-\mathrm{S}(1)-\mathrm{N}(2)-\mathrm{C}(17)$ & $101.1(9)$ \\
$\mathrm{O}(6)-\mathrm{S}(2)-\mathrm{N}(4)-\mathrm{C}(41)$ & $12.5(12)$ \\
$\mathrm{O}(5)-\mathrm{S}(2)-\mathrm{N}(4)-\mathrm{C}(41)$ & $153.6(9)$ \\
$\mathrm{C}(48)-\mathrm{S}(2)-\mathrm{N}(4)-\mathrm{C}(41)$ & $-100.2(8)$ \\
$\mathrm{C}(6)-\mathrm{C}(1)-\mathrm{C}(2)-\mathrm{C}(3)$ & $0.1(15)$ \\
$\mathrm{C}(7)-\mathrm{C}(1)-\mathrm{C}(2)-\mathrm{C}(3)$ & $175.9(10)$ \\
$\mathrm{C}(6)-\mathrm{C}(1)-\mathrm{C}(2)-\mathrm{Br}(1)$ & $-177.4(7)$ \\
$\mathrm{C}(7)-\mathrm{C}(1)-\mathrm{C}(2)-\mathrm{Br}(1)$ & $-1.6(12)$ \\
$\mathrm{C}(1)-\mathrm{C}(2)-\mathrm{C}(3)-\mathrm{C}(4)$ & $-1.8(17)$ \\
$\mathrm{Br}(1)-\mathrm{C}(2)-\mathrm{C}(3)-\mathrm{C}(4)$ & $175.8(9)$ \\
$\mathrm{C}(2)-\mathrm{C}(3)-\mathrm{C}(4)-\mathrm{C}(5)$ & $3.2(18)$ \\
$\mathrm{C}(3)-\mathrm{C}(4)-\mathrm{C}(5)-\mathrm{C}(6)$ & $-2.9(18)$ \\
$\mathrm{C}(2)-\mathrm{C}(1)-\mathrm{C}(6)-\mathrm{C}(5)$ & $0.2(14)$ \\
$\mathrm{C}(7)-\mathrm{C}(1)-\mathrm{C}(6)-\mathrm{C}(5)$ & $-175.8(9)$ \\
$\mathrm{C}(4)-\mathrm{C}(5)-\mathrm{C}(6)-\mathrm{C}(1)$ & $1.2(16)$ \\
$\mathrm{C}(8)-\mathrm{N}(1)-\mathrm{C}(7)-\mathrm{C}(1)$ & $-74.4(11)$ \\
$\mathrm{C}(6)-\mathrm{C}(1)-\mathrm{C}(7)-\mathrm{N}(1)$ & $107.9(9)$ \\
$\mathrm{C}(2)-\mathrm{C}(1)-\mathrm{C}(7)-\mathrm{N}(1)$ & $-67.7(11)$ \\
$\mathrm{C}(7)-\mathrm{N}(1)-\mathrm{C}(8)-\mathrm{O}(1)$ & $-10.7(13)$ \\
$\mathrm{C}(7)-\mathrm{N}(1)-\mathrm{C}(8)-\mathrm{C}(9)$ & $165.8(8)$ \\
$\mathrm{O}(1)-\mathrm{C}(8)-\mathrm{C}(9)-\mathrm{C}(10)$ & $74.4(10)$ \\
$\mathrm{N}(1)-\mathrm{C}(8)-\mathrm{C}(9)-\mathrm{C}(10)$ & $-102.2(8)$ \\
$\mathrm{O}(1)-\mathrm{C}(8)-\mathrm{C}(9)-\mathrm{C}(16)$ & $-164.4(8)$ \\
$\mathrm{N}(1)-\mathrm{C}(8)-\mathrm{C}(9)-\mathrm{C}(16)$ & $19.0(11)$ \\
$\mathrm{O}(1)-\mathrm{C}(8)-\mathrm{C}(9)-\mathrm{C}(17)$ & $-46.1(10)$ \\
$\mathrm{N}(1)-\mathrm{C}(8)-\mathrm{C}(9)-\mathrm{C}(17)$ & $137.4(7)$ \\
$\mathrm{C}(8)-\mathrm{C}(9)-\mathrm{C}(10)-\mathrm{C}(15)$ & $-169.5(11)$ \\
$\mathrm{C}(16)-\mathrm{C}(9)-\mathrm{C}(10)-\mathrm{C}(15)$ & $66.8(14)$ \\
$\mathrm{C}(17)-\mathrm{C}(9)-\mathrm{C}(10)-\mathrm{C}(15)$ & $-48.7(13)$ \\
$\mathrm{C}(8)-\mathrm{C}(9)-\mathrm{C}(10)-\mathrm{C}(11)$ & $9.5(12)$ \\
$\mathrm{C}(16)-\mathrm{C}(9)-\mathrm{C}(10)-\mathrm{C}(11)$ & $-114.2(10)$ \\
$\mathrm{C}(17)-\mathrm{C}(9)-\mathrm{C}(10)-\mathrm{C}(11)$ & \\
& $130.2(10)$ \\
&
\end{tabular}




$\begin{array}{lc}\mathrm{C}(15)-\mathrm{C}(10)-\mathrm{C}(11)-\mathrm{C}(12) & -4.0(19) \\ \mathrm{C}(9)-\mathrm{C}(10)-\mathrm{C}(11)-\mathrm{C}(12) & 177.0(11) \\ \mathrm{C}(10)-\mathrm{C}(11)-\mathrm{C}(12)-\mathrm{C}(13) & 4(2) \\ \mathrm{C}(11)-\mathrm{C}(12)-\mathrm{C}(13)-\mathrm{C}(14) & -3(3) \\ \mathrm{C}(12)-\mathrm{C}(13)-\mathrm{C}(14)-\mathrm{C}(15) & 4(3) \\ \mathrm{C}(11)-\mathrm{C}(10)-\mathrm{C}(15)-\mathrm{C}(14) & 4(2) \\ \mathrm{C}(9)-\mathrm{C}(10)-\mathrm{C}(15)-\mathrm{C}(14) & -176.6(16) \\ \mathrm{C}(13)-\mathrm{C}(14)-\mathrm{C}(15)-\mathrm{C}(10) & -4(3) \\ \mathrm{S}(1)-\mathrm{N}(2)-\mathrm{C}(17)-\mathrm{C}(18) & -99.1(8) \\ \mathrm{S}(1)-\mathrm{N}(2)-\mathrm{C}(17)-\mathrm{C}(9) & 133.8(7) \\ \mathrm{C}(10)-\mathrm{C}(9)-\mathrm{C}(17)-\mathrm{N}(2) & -58.9(8) \\ \mathrm{C}(8)-\mathrm{C}(9)-\mathrm{C}(17)-\mathrm{N}(2) & 59.8(8) \\ \mathrm{C}(16)-\mathrm{C}(9)-\mathrm{C}(17)-\mathrm{N}(2) & -177.0(7) \\ \mathrm{C}(10)-\mathrm{C}(9)-\mathrm{C}(17)-\mathrm{C}(18) & 176.6(7) \\ \mathrm{C}(8)-\mathrm{C}(9)-\mathrm{C}(17)-\mathrm{C}(18) & -64.7(8) \\ \mathrm{C}(16)-\mathrm{C}(9)-\mathrm{C}(17)-\mathrm{C}(18) & 58.5(9) \\ \mathrm{N}(2)-\mathrm{C}(17)-\mathrm{C}(18)-\mathrm{C}(19) & -49.8(11) \\ \mathrm{C}(9)-\mathrm{C}(17)-\mathrm{C}(18)-\mathrm{C}(19) & 72.0(10) \\ \mathrm{N}(2)-\mathrm{C}(17)-\mathrm{C}(18)-\mathrm{C}(23) & 127.9(9) \\ \mathrm{C}(9)-\mathrm{C}(17)-\mathrm{C}(18)-\mathrm{C}(23) & -110.3(9) \\ \mathrm{C}(23)-\mathrm{C}(18)-\mathrm{C}(19)-\mathrm{C}(20) & 0.5(14) \\ \mathrm{C}(17)-\mathrm{C}(18)-\mathrm{C}(19)-\mathrm{C}(20) & 178.1(9) \\ \mathrm{C}(18)-\mathrm{C}(19)-\mathrm{C}(20)-\mathrm{C}(21) & -0.7(17) \\ \mathrm{C}(19)-\mathrm{C}(20)-\mathrm{C}(21)-\mathrm{C}(22) & 1(2) \\ \mathrm{C}(20)-\mathrm{C}(21)-\mathrm{C}(22)-\mathrm{C}(23) & -2(2) \\ \mathrm{C}(21)-\mathrm{C}(22)-\mathrm{C}(23)-\mathrm{C}(18) & 2(2) \\ \mathrm{C}(19)-\mathrm{C}(18)-\mathrm{C}(23)-\mathrm{C}(22) & -1.0(16) \\ \mathrm{C}(17)-\mathrm{C}(18)-\mathrm{C}(23)-\mathrm{C}(22) & -178.8(10) \\ \mathrm{O}(2)-\mathrm{S}(1)-\mathrm{C}(24)-\mathrm{F}(2) & 176.1(11) \\ \mathrm{O}(3)-\mathrm{S}(1)-\mathrm{C}(24)-\mathrm{F}(2) & -54.7(12) \\ \mathrm{N}(2)-\mathrm{S}(1)-\mathrm{C}(24)-\mathrm{F}(2) & 58.5(12) \\ \mathrm{O}(2)-\mathrm{S}(1)-\mathrm{C}(24)-\mathrm{F}(1) & 53.5(11) \\ \mathrm{O}(3)-\mathrm{S}(1)-\mathrm{C}(24)-\mathrm{F}(1) & -177.3(9) \\ \mathrm{N}(2)-\mathrm{S}(1)-\mathrm{C}(24)-\mathrm{F}(1) & -64.1(11) \\ \mathrm{O}(2)-\mathrm{S}(1)-\mathrm{C}(24)-\mathrm{F}(3) & \\ \mathrm{O}(3)-\mathrm{S}(1)-\mathrm{C}(24)-\mathrm{F}(3) & \\ & \end{array}$




\begin{tabular}{|c|c|}
\hline$N(2)-S(1)-C(24)-F(3)$ & $178.5(8)$ \\
\hline$C(30)-C(25)-C(26)-C(27)$ & $0.7(15)$ \\
\hline$C(31)-C(25)-C(26)-C(27)$ & $-179.7(10)$ \\
\hline$C(30)-C(25)-C(26)-B r(2)$ & $-179.5(7)$ \\
\hline$C(31)-C(25)-C(26)-B r(2)$ & $0.1(13)$ \\
\hline$C(25)-C(26)-C(27)-C(28)$ & $-1.1(17)$ \\
\hline $\mathrm{Br}(2)-\mathrm{C}(26)-\mathrm{C}(27)-\mathrm{C}(28)$ & $179.1(9)$ \\
\hline$C(26)-C(27)-C(28)-C(29)$ & $2.9(17)$ \\
\hline$C(27)-C(28)-C(29)-C(30)$ & $-4.5(18)$ \\
\hline$C(28)-C(29)-C(30)-C(25)$ & $4.1(17)$ \\
\hline$C(26)-C(25)-C(30)-C(29)$ & $-2.2(14)$ \\
\hline$C(31)-C(25)-C(30)-C(29)$ & $178.2(9)$ \\
\hline $\mathrm{C}(32)-\mathrm{N}(3)-\mathrm{C}(31)-\mathrm{C}(25)$ & $78.6(11)$ \\
\hline$C(26)-C(25)-C(31)-N(3)$ & $73.5(12)$ \\
\hline $\mathrm{C}(30)-\mathrm{C}(25)-\mathrm{C}(31)-\mathrm{N}(3)$ & $-106.9(11)$ \\
\hline $\mathrm{C}(31)-\mathrm{N}(3)-\mathrm{C}(32)-\mathrm{O}(4)$ & $1.2(13)$ \\
\hline $\mathrm{C}(31)-\mathrm{N}(3)-\mathrm{C}(32)-\mathrm{C}(33)$ & $179.8(8)$ \\
\hline $\mathrm{O}(4)-\mathrm{C}(32)-\mathrm{C}(33)-\mathrm{C}(40)$ & $-162.4(8)$ \\
\hline $\mathrm{N}(3)-\mathrm{C}(32)-\mathrm{C}(33)-\mathrm{C}(40)$ & 19.1(11) \\
\hline $\mathrm{O}(4)-\mathrm{C}(32)-\mathrm{C}(33)-\mathrm{C}(34)$ & $75.5(10)$ \\
\hline $\mathrm{N}(3)-\mathrm{C}(32)-\mathrm{C}(33)-\mathrm{C}(34)$ & $-103.0(8)$ \\
\hline $\mathrm{O}(4)-\mathrm{C}(32)-\mathrm{C}(33)-\mathrm{C}(41)$ & $-42.1(12)$ \\
\hline $\mathrm{N}(3)-\mathrm{C}(32)-\mathrm{C}(33)-\mathrm{C}(41)$ & $139.3(8)$ \\
\hline$C(40)-C(33)-C(34)-C(35)$ & $-121.5(10)$ \\
\hline$C(32)-C(33)-C(34)-C(35)$ & $1.4(12)$ \\
\hline$C(41)-C(33)-C(34)-C(35)$ & $118.2(10)$ \\
\hline$C(40)-C(33)-C(34)-C(39)$ & $54.9(10)$ \\
\hline$C(32)-C(33)-C(34)-C(39)$ & $177.9(8)$ \\
\hline$C(41)-C(33)-C(34)-C(39)$ & $-65.3(10)$ \\
\hline$C(39)-C(34)-C(35)-C(36)$ & $0.7(15)$ \\
\hline$C(33)-C(34)-C(35)-C(36)$ & $177.2(9)$ \\
\hline$C(34)-C(35)-C(36)-C(37)$ & $-1.1(17)$ \\
\hline$C(35)-C(36)-C(37)-C(38)$ & $1.0(17)$ \\
\hline$C(36)-C(37)-C(38)-C(39)$ & $-0.6(17)$ \\
\hline $\mathrm{C}(37)-\mathrm{C}(38)-\mathrm{C}(39)-\mathrm{C}(34)$ & $0.2(17)$ \\
\hline $\mathrm{C}(35)-\mathrm{C}(34)-\mathrm{C}(39)-\mathrm{C}(38)$ & $-0.3(15)$ \\
\hline
\end{tabular}




$\begin{array}{lc}\mathrm{C}(33)-\mathrm{C}(34)-\mathrm{C}(39)-\mathrm{C}(38) & -176.9(9) \\ \mathrm{S}(2)-\mathrm{N}(4)-\mathrm{C}(41)-\mathrm{C}(42) & -83.1(10) \\ \mathrm{S}(2)-\mathrm{N}(4)-\mathrm{C}(41)-\mathrm{C}(33) & 149.5(7) \\ \mathrm{C}(40)-\mathrm{C}(33)-\mathrm{C}(41)-\mathrm{N}(4) & 179.6(8) \\ \mathrm{C}(32)-\mathrm{C}(33)-\mathrm{C}(41)-\mathrm{N}(4) & 57.3(10) \\ \mathrm{C}(34)-\mathrm{C}(33)-\mathrm{C}(41)-\mathrm{N}(4) & -59.0(9) \\ \mathrm{C}(40)-\mathrm{C}(33)-\mathrm{C}(41)-\mathrm{C}(42) & 51.8(10) \\ \mathrm{C}(32)-\mathrm{C}(33)-\mathrm{C}(41)-\mathrm{C}(42) & -70.5(10) \\ \mathrm{C}(34)-\mathrm{C}(33)-\mathrm{C}(41)-\mathrm{C}(42) & 173.2(7) \\ \mathrm{N}(4)-\mathrm{C}(41)-\mathrm{C}(42)-\mathrm{C}(47) & 114.8(12) \\ \mathrm{C}(33)-\mathrm{C}(41)-\mathrm{C}(42)-\mathrm{C}(47) & -119.3(12) \\ \mathrm{N}(4)-\mathrm{C}(41)-\mathrm{C}(42)-\mathrm{C}(43) & -60.8(13) \\ \mathrm{C}(33)-\mathrm{C}(41)-\mathrm{C}(42)-\mathrm{C}(43) & 65.1(13) \\ \mathrm{C}(47)-\mathrm{C}(42)-\mathrm{C}(43)-\mathrm{C}(44) & 2.1(18) \\ \mathrm{C}(41)-\mathrm{C}(42)-\mathrm{C}(43)-\mathrm{C}(44) & 177.7(10) \\ \mathrm{C}(42)-\mathrm{C}(43)-\mathrm{C}(44)-\mathrm{C}(45) & -3(2) \\ \mathrm{C}(43)-\mathrm{C}(44)-\mathrm{C}(45)-\mathrm{C}(46) & -1(2) \\ \mathrm{C}(44)-\mathrm{C}(45)-\mathrm{C}(46)-\mathrm{C}(47) & 4(3) \\ \mathrm{C}(45)-\mathrm{C}(46)-\mathrm{C}(47)-\mathrm{C}(42) & -5(3) \\ \mathrm{C}(43)-\mathrm{C}(42)-\mathrm{C}(47)-\mathrm{C}(46) & 2(2) \\ \mathrm{C}(41)-\mathrm{C}(42)-\mathrm{C}(47)-\mathrm{C}(46) & -174.3(14) \\ \mathrm{O}(6)-\mathrm{S}(2)-\mathrm{C}(48)-\mathrm{F}(5) & -53.2(12) \\ \mathrm{O}(5)-\mathrm{S}(2)-\mathrm{C}(48)-\mathrm{F}(5) & 175.9(10) \\ \mathrm{N}(4)-\mathrm{S}(2)-\mathrm{C}(48)-\mathrm{F}(5) & 63.4(11) \\ \mathrm{O}(6)-\mathrm{S}(2)-\mathrm{C}(48)-\mathrm{F}(4) & -175.3(10) \\ \mathrm{O}(5)-\mathrm{S}(2)-\mathrm{C}(48)-\mathrm{F}(4) & 53.8(10) \\ \mathrm{N}(4)-\mathrm{S}(2)-\mathrm{C}(48)-\mathrm{F}(4) & -58.7(10) \\ \mathrm{O}(6)-\mathrm{S}(2)-\mathrm{C}(48)-\mathrm{F}(6) & 62.5(10) \\ \mathrm{O}(5)-\mathrm{S}(2)-\mathrm{C}(48)-\mathrm{F}(6) & -68.4(9) \\ \mathrm{N}(4)-\mathrm{S}(2)-\mathrm{C}(48)-\mathrm{F}(6) & 179.1(8) \\ & \end{array}$


Structure 2. Crystals of the $\beta$-lactam product of Table 1 , entry 8 that were suitable for X-ray structural analysis were obtained by dissolving the $\beta$-lactam in benzene and adding pentane at $0{ }^{\circ} \mathrm{C}$.

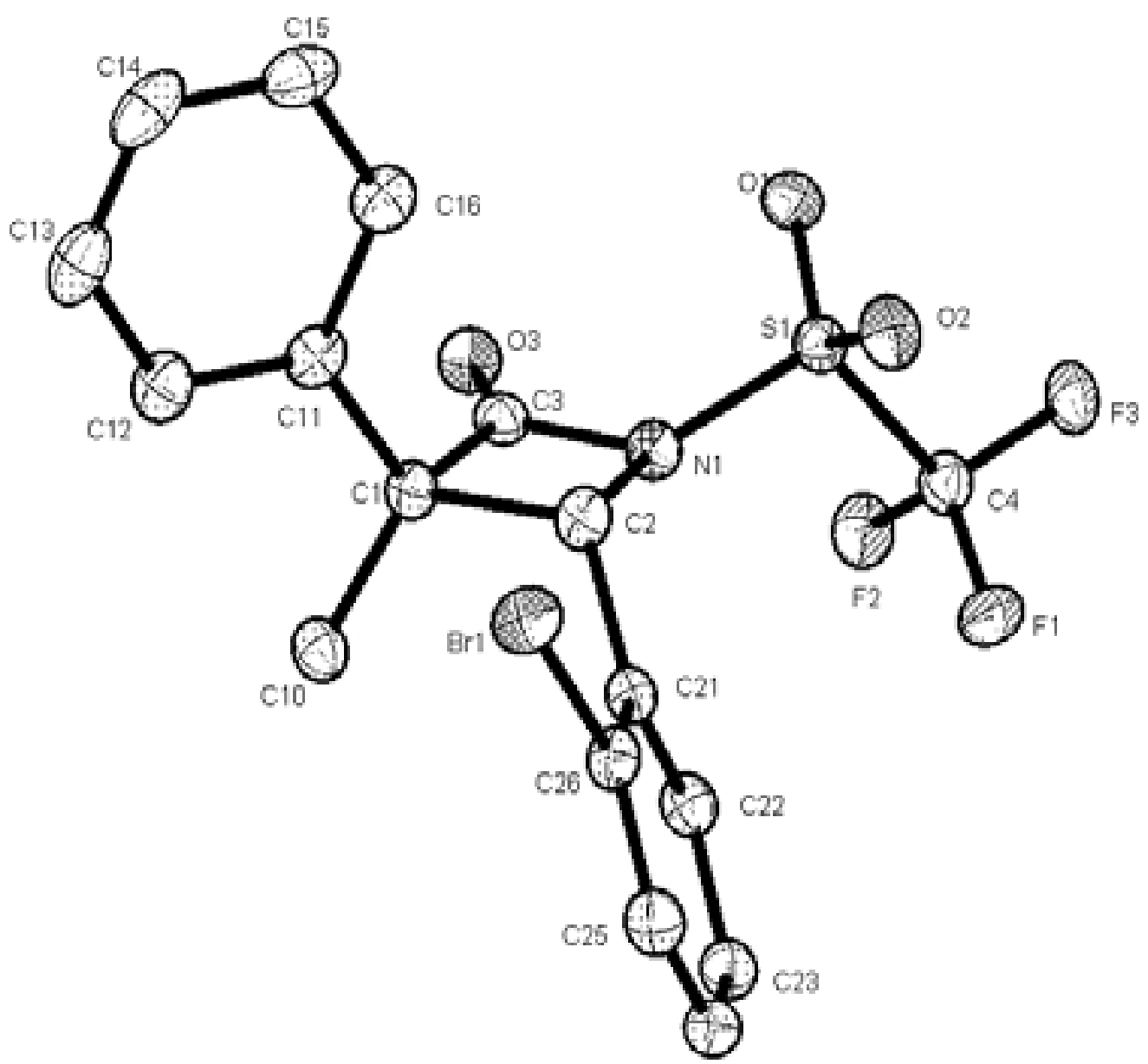

$\mathrm{C} 24$ 
Table 1. Crystal data and structure refinement.

Identification code

Empirical formula

Formula weight

Temperature

Wavelength

Crystal system

Space group

Unit cell dimensions

Volume

Z

Density (calculated)

Absorption coefficient

$\mathrm{F}(000)$

Crystal size

Theta range for data collection

Index ranges

Reflections collected

Independent reflections

Completeness to theta $=26.37^{\circ}$

Absorption correction

Max. and min. transmission

Refinement method

Data / restraints / parameters

Goodness-of-fit on $\mathrm{F}^{2}$

Final $\mathrm{R}$ indices [I>2sigma(I)]

$\mathrm{R}$ indices (all data)

Absolute structure parameter

Largest diff. peak and hole
Table 1, Entry 8

$\mathrm{C}_{17} \mathrm{H}_{13} \mathrm{BrF}_{3} \mathrm{NO}_{3} \mathrm{~S}$

448.25

100(2) K

$0.71073 \AA$

Orthorhombic

$\mathrm{P} 2{ }_{1} 2_{1} 2_{1}$

$a=7.2080(3) \AA \quad \quad=90^{\circ}$.

$\mathrm{b}=8.7647(3) \AA \quad \quad=90^{\circ}$.

$\mathrm{c}=27.1374(11) \AA \quad \quad=90^{\circ}$.

1714.43(12) $\AA^{3}$

4

$1.737 \mathrm{Mg} / \mathrm{m}^{3}$

$2.568 \mathrm{~mm}^{-1}$

896

$0.20 \times 0.20 \times 0.10 \mathrm{~mm}^{3}$

2.44 to $26.37^{\circ}$.

$-8<=\mathrm{h}<=9,0<=\mathrm{k}<=10,0<=\mathrm{l}<=33$

26769

$3480[\mathrm{R}(\mathrm{int})=0.0329]$

$99.9 \%$

Semi-empirical from equivalents

0.7833 and 0.6276

Full-matrix least-squares on $\mathrm{F}^{2}$

3480 / 0 / 236

1.059

$\mathrm{R} 1=0.0260, \mathrm{wR} 2=0.0653$

$\mathrm{R} 1=0.0272, \mathrm{wR} 2=0.0657$

$0.024(6)$

0.691 and -0.276 e. $\AA^{-3}$ 
Table 2. Atomic coordinates $\left(\times 10^{4}\right)$ and equivalent isotropic displacement parameters $\left(\AA^{2} \times 10^{3}\right)$ for Table 1, Entry 8. U(eq) is defined as one third of the trace of the orthogonalized $\mathrm{Uij}^{\mathrm{ij}}$ tensor.

\begin{tabular}{|c|c|c|c|c|}
\hline & $x$ & $\mathrm{y}$ & z & $\mathrm{U}(\mathrm{eq})$ \\
\hline $\mathrm{N}(1)$ & $-4399(3)$ & $-4545(2)$ & $1849(1)$ & $18(1)$ \\
\hline$S(1)$ & $-2700(1)$ & $-5527(1)$ & 2099(1) & $17(1)$ \\
\hline $\mathrm{O}(1)$ & $-3468(3)$ & $-6760(2)$ & $2373(1)$ & $23(1)$ \\
\hline $\mathrm{O}(2)$ & $-1314(3)$ & $-5738(2)$ & $1735(1)$ & $24(1)$ \\
\hline$C(4)$ & $-1777(4)$ & $-4213(3)$ & $2568(1)$ & $20(1)$ \\
\hline $\mathrm{F}(1)$ & $-888(2)$ & $-3063(2)$ & $2362(1)$ & $26(1)$ \\
\hline $\mathrm{F}(2)$ & $-3176(2)$ & $-3681(2)$ & $2837(1)$ & $26(1)$ \\
\hline $\mathrm{F}(3)$ & $-634(2)$ & $-4977(2)$ & $2858(1)$ & $25(1)$ \\
\hline$C(1)$ & $-6718(4)$ & $-4183(3)$ & 1371(1) & $16(1)$ \\
\hline$C(10)$ & $-7810(4)$ & $-2692(3)$ & $1359(1)$ & $20(1)$ \\
\hline$C(11)$ & $-7465(4)$ & $-5436(3)$ & $1036(1)$ & $19(1)$ \\
\hline$C(12)$ & $-8898(4)$ & $-5168(3)$ & $706(1)$ & $23(1)$ \\
\hline$C(13)$ & $-9524(4)$ & $-6322(3)$ & 399(1) & $29(1)$ \\
\hline$C(14)$ & $-8730(4)$ & $-7752(3)$ & $412(1)$ & $30(1)$ \\
\hline$C(15)$ & $-7311(4)$ & $-8046(3)$ & $743(1)$ & $28(1)$ \\
\hline$C(16)$ & $-6684(4)$ & $-6893(3)$ & $1055(1)$ & $23(1)$ \\
\hline$C(2)$ & $-4516(4)$ & $-4008(3)$ & $1328(1)$ & $17(1)$ \\
\hline$C(21)$ & $-3748(4)$ & $-2432(3)$ & $1235(1)$ & $17(1)$ \\
\hline$C(22)$ & $-3550(4)$ & $-1377(3)$ & $1618(1)$ & $19(1)$ \\
\hline$C(23)$ & $-2897(4)$ & $88(3)$ & $1530(1)$ & $21(1)$ \\
\hline$C(24)$ & $-2411(4)$ & $525(3)$ & 1057(1) & $22(1)$ \\
\hline$C(25)$ & $-2598(4)$ & $-500(3)$ & $666(1)$ & $22(1)$ \\
\hline$C(26)$ & $-3280(4)$ & $-1955(3)$ & 763(1) & $18(1)$ \\
\hline $\operatorname{Br}(1)$ & $-3610(1)$ & $-3295(1)$ & $214(1)$ & $24(1)$ \\
\hline$C(3)$ & $-6367(4)$ & $-4755(3)$ & 1899(1) & $17(1)$ \\
\hline $\mathrm{O}(3)$ & $-7253(3)$ & $-5202(2)$ & $2237(1)$ & $21(1)$ \\
\hline
\end{tabular}


Table 3. Bond lengths $[\AA ̊]$ and angles $\left[{ }^{\circ}\right]$ for Table 1, Entry 8.

\begin{tabular}{ll}
\hline $\mathrm{N}(1)-\mathrm{C}(3)$ & $1.437(4)$ \\
$\mathrm{N}(1)-\mathrm{C}(2)$ & $1.494(3)$ \\
$\mathrm{N}(1)-\mathrm{S}(1)$ & $1.642(2)$ \\
$\mathrm{S}(1)-\mathrm{O}(2)$ & $1.417(2)$ \\
$\mathrm{S}(1)-\mathrm{O}(1)$ & $1.4250(18)$ \\
$\mathrm{S}(1)-\mathrm{C}(4)$ & $1.842(3)$ \\
$\mathrm{C}(4)-\mathrm{F}(1)$ & $1.319(3)$ \\
$\mathrm{C}(4)-\mathrm{F}(3)$ & $1.322(3)$ \\
$\mathrm{C}(4)-\mathrm{F}(2)$ & $1.329(3)$ \\
$\mathrm{C}(1)-\mathrm{C}(11)$ & $1.524(3)$ \\
$\mathrm{C}(1)-\mathrm{C}(10)$ & $1.526(3)$ \\
$\mathrm{C}(1)-\mathrm{C}(3)$ & $1.537(3)$ \\
$\mathrm{C}(1)-\mathrm{C}(2)$ & $1.599(4)$ \\
$\mathrm{C}(11)-\mathrm{C}(12)$ & $1.387(4)$ \\
$\mathrm{C}(11)-\mathrm{C}(16)$ & $1.396(4)$ \\
$\mathrm{C}(12)-\mathrm{C}(13)$ & $1.386(4)$ \\
$\mathrm{C}(13)-\mathrm{C}(14)$ & $1.377(4)$ \\
$\mathrm{C}(14)-\mathrm{C}(15)$ & $1.386(4)$ \\
$\mathrm{C}(15)-\mathrm{C}(16)$ & $1.394(4)$ \\
$\mathrm{C}(2)-\mathrm{C}(21)$ & $1.509(3)$ \\
$\mathrm{C}(21)-\mathrm{C}(26)$ & $1.391(3)$ \\
$\mathrm{C}(21)-\mathrm{C}(22)$ & $1.397(3)$ \\
$\mathrm{C}(22)-\mathrm{C}(23)$ & $1.388(4)$ \\
$\mathrm{C}(23)-\mathrm{C}(24)$ & $1.384(4)$ \\
$\mathrm{C}(24)-\mathrm{C}(25)$ & $1.399(4)$ \\
$\mathrm{C}(25)-\mathrm{C}(26)$ & $1.391(4)$ \\
$\mathrm{C}(26)-\mathrm{Br}(1)$ & $1.911(2)$ \\
$\mathrm{C}(3)-\mathrm{O}(3)$ & $1.185(3)$ \\
$\mathrm{C}(3)-\mathrm{N}(1)-\mathrm{C}(2)$ & $94.17(19)$ \\
$\mathrm{C}(3)-\mathrm{N}(1)-\mathrm{S}(1)$ & $129.08(17)$ \\
$\mathrm{C}(2)-\mathrm{N}(1)-\mathrm{S}(1)$ & $126.68(18)$ \\
$\mathrm{O}(2)-\mathrm{S}(1)-\mathrm{O}(1)$ & $122.66(11)$ \\
$\mathrm{O}(2)-\mathrm{S}(1)-\mathrm{N}(1)$ & $107.88(11)$ \\
& \\
& \\
& \\
& \\
& \\
&
\end{tabular}




$\begin{array}{ll}\mathrm{O}(1)-\mathrm{S}(1)-\mathrm{N}(1) & 108.90(11) \\ \mathrm{O}(2)-\mathrm{S}(1)-\mathrm{C}(4) & 107.99(12) \\ \mathrm{O}(1)-\mathrm{S}(1)-\mathrm{C}(4) & 104.62(11) \\ \mathrm{N}(1)-\mathrm{S}(1)-\mathrm{C}(4) & 103.08(11) \\ \mathrm{F}(1)-\mathrm{C}(4)-\mathrm{F}(3) & 109.7(2) \\ \mathrm{F}(1)-\mathrm{C}(4)-\mathrm{F}(2) & 109.5(2) \\ \mathrm{F}(3)-\mathrm{C}(4)-\mathrm{F}(2) & 108.9(2) \\ \mathrm{F}(1)-\mathrm{C}(4)-\mathrm{S}(1) & 111.06(18) \\ \mathrm{F}(3)-\mathrm{C}(4)-\mathrm{S}(1) & 108.68(16) \\ \mathrm{F}(2)-\mathrm{C}(4)-\mathrm{S}(1) & 108.97(17) \\ \mathrm{C}(11)-\mathrm{C}(1)-\mathrm{C}(10) & 115.0(2) \\ \mathrm{C}(11)-\mathrm{C}(1)-\mathrm{C}(3) & 112.23(19) \\ \mathrm{C}(10)-\mathrm{C}(1)-\mathrm{C}(3) & 112.6(2) \\ \mathrm{C}(11)-\mathrm{C}(1)-\mathrm{C}(2) & 112.1(2) \\ \mathrm{C}(10)-\mathrm{C}(1)-\mathrm{C}(2) & 115.4(2) \\ \mathrm{C}(3)-\mathrm{C}(1)-\mathrm{C}(2) & 86.37(18) \\ \mathrm{C}(12)-\mathrm{C}(11)-\mathrm{C}(16) & 118.6(2) \\ \mathrm{C}(12)-\mathrm{C}(11)-\mathrm{C}(1) & 121.7(2) \\ \mathrm{C}(16)-\mathrm{C}(11)-\mathrm{C}(1) & 119.6(2) \\ \mathrm{C}(13)-\mathrm{C}(12)-\mathrm{C}(11) & 120.4(3) \\ \mathrm{C}(14)-\mathrm{C}(13)-\mathrm{C}(12) & 120.9(3) \\ \mathrm{C}(13)-\mathrm{C}(14)-\mathrm{C}(15) & 119.5(3) \\ \mathrm{C}(14)-\mathrm{C}(15)-\mathrm{C}(16) & 119.9(3) \\ \mathrm{C}(15)-\mathrm{C}(16)-\mathrm{C}(11) & 120.6(3) \\ \mathrm{N}(1)-\mathrm{C}(2)-\mathrm{C}(21) & 115.2(2) \\ \mathrm{N}(1)-\mathrm{C}(2)-\mathrm{C}(1) & 87.47(19) \\ \mathrm{C}(21)-\mathrm{C}(2)-\mathrm{C}(1) & 117.7(2) \\ \mathrm{C}(26)-\mathrm{C}(21)-\mathrm{C}(22) & 117.4(2) \\ \mathrm{C}(26)-\mathrm{C}(21)-\mathrm{C}(2) & 121.2(2) \\ \mathrm{C}(22)-\mathrm{C}(21)-\mathrm{C}(2) & 121.3(2) \\ \mathrm{C}(23)-\mathrm{C}(22)-\mathrm{C}(21) & 121.4(2) \\ \mathrm{C}(24)-\mathrm{C}(23)-\mathrm{C}(22) & 120.0(2) \\ \mathrm{C}(23)-\mathrm{C}(24)-\mathrm{C}(25) & 120.2(2) \\ \mathrm{C}(26)-\mathrm{C}(25)-\mathrm{C}(24) & 118.6(2) \\ \mathrm{C}(21)-\mathrm{C}(26)-\mathrm{C}(25) & 122.4(2) \\ \mathrm{C}(21)-\mathrm{C}(26)-\mathrm{Br}(1) & 120.19(18) \\ & \end{array}$




$\begin{array}{ll}\mathrm{C}(25)-\mathrm{C}(26)-\mathrm{Br}(1) & 117.37(19) \\ \mathrm{O}(3)-\mathrm{C}(3)-\mathrm{N}(1) & 130.3(2) \\ \mathrm{O}(3)-\mathrm{C}(3)-\mathrm{C}(1) & 137.8(3) \\ \mathrm{N}(1)-\mathrm{C}(3)-\mathrm{C}(1) & 91.95(19)\end{array}$

Symmetry transformations used to generate equivalent atoms: 
Table 4. Anisotropic displacement parameters $\left(\AA^{2} \times 10^{3}\right)$ for Table 1, Entry 8 . The anisotropic displacement factor exponent takes the form: $-2_{-}^{2}\left[h^{2} a^{* 2} U^{11}+\ldots+2 h k\right.$ $a^{*} b^{*} U^{12}$ ]

\begin{tabular}{|c|c|c|c|c|c|c|}
\hline & U11 & $\mathrm{U}^{22}$ & U33 & $\mathrm{U}^{23}$ & U13 & U12 \\
\hline $\mathrm{N}(1)$ & $20(1)$ & $20(1)$ & $12(1)$ & $3(1)$ & $-1(1)$ & $-1(1)$ \\
\hline$S(1)$ & 18(1) & $17(1)$ & $17(1)$ & $0(1)$ & $-1(1)$ & $1(1)$ \\
\hline $\mathrm{O}(1)$ & $25(1)$ & 19(1) & $23(1)$ & $4(1)$ & $-3(1)$ & $0(1)$ \\
\hline $\mathrm{O}(2)$ & $22(1)$ & $30(1)$ & $20(1)$ & $-2(1)$ & $1(1)$ & $4(1)$ \\
\hline$C(4)$ & 19(1) & 21(1) & $18(1)$ & $-2(1)$ & $-3(1)$ & $1(1)$ \\
\hline $\mathrm{F}(1)$ & $27(1)$ & 21(1) & $31(1)$ & $3(1)$ & $-3(1)$ & $-6(1)$ \\
\hline $\mathrm{F}(2)$ & $23(1)$ & $31(1)$ & $24(1)$ & $-9(1)$ & $2(1)$ & $3(1)$ \\
\hline $\mathrm{F}(3)$ & $23(1)$ & $29(1)$ & $23(1)$ & $2(1)$ & $-8(1)$ & $1(1)$ \\
\hline$C(1)$ & 18(1) & 19(1) & 13(1) & $0(1)$ & $-1(1)$ & $0(1)$ \\
\hline$C(10)$ & )20(1) & $22(1)$ & $18(1)$ & $0(1)$ & $-2(1)$ & $2(1)$ \\
\hline$C(11)$ & )20(1) & $22(1)$ & $14(1)$ & $-1(1)$ & $4(1)$ & $-4(1)$ \\
\hline$C(12)$ & )26(2) & $28(1)$ & $17(1)$ & $0(1)$ & $-2(1)$ & $-2(1)$ \\
\hline$C(13)$ & $27(2)$ & $41(2)$ & $18(1)$ & $-2(1)$ & $-3(1)$ & $-11(1)$ \\
\hline$C(14)$ & $30(2)$ & $35(1)$ & $25(1)$ & $-14(1)$ & $7(1)$ & $-14(1)$ \\
\hline$C(15)$ & )31(2) & $21(1)$ & $33(2)$ & $-6(1)$ & $9(1)$ & $-4(1)$ \\
\hline$C(16)$ & )24(1) & $24(1)$ & $22(1)$ & $-4(1)$ & $2(1)$ & $-1(1)$ \\
\hline$C(2)$ & $20(1)$ & $22(1)$ & $9(1)$ & $0(1)$ & $0(1)$ & $-2(1)$ \\
\hline$C(21)$ & )16(1) & $18(1)$ & $18(1)$ & 1(1) & $-2(1)$ & $1(1)$ \\
\hline$C(22)$ & )21(1) & $22(1)$ & $15(1)$ & 1(1) & $-1(1)$ & $2(1)$ \\
\hline$C(23)$ & $21(1)$ & $20(1)$ & $23(1)$ & $-4(1)$ & $-2(1)$ & $2(1)$ \\
\hline$C(24)$ & 20(1) & 19(1) & $28(1)$ & $2(1)$ & $-2(1)$ & $0(1)$ \\
\hline$C(25)$ & )22(1) & $25(1)$ & $17(1)$ & $5(1)$ & $1(1)$ & $0(1)$ \\
\hline$C(26)$ & )17(1) & $21(1)$ & $16(1)$ & $-3(1)$ & $0(1)$ & $0(1)$ \\
\hline $\operatorname{Br}(1)$ & $33(1)$ & $26(1)$ & $14(1)$ & $-1(1)$ & $0(1)$ & $-4(1)$ \\
\hline$C(3)$ & $20(1)$ & $14(1)$ & $17(1)$ & $-2(1)$ & $-2(1)$ & $2(1)$ \\
\hline $\mathrm{O}(3)$ & $22(1)$ & $24(1)$ & $17(1)$ & $1(1)$ & $3(1)$ & $-1(1)$ \\
\hline
\end{tabular}


Table 5. Hydrogen coordinates ( $\left.x 10^{4}\right)$ and isotropic displacement parameters $\left(\AA^{2} \mathrm{x}\right.$ $10^{3}$ ) for Table 1, Entry 8.

\begin{tabular}{lrrrl}
\hline & $x$ & $y$ & $z$ & $U(e q)$ \\
\hline$H(10 A)$ & -7694 & -2225 & 1033 & 30 \\
$\mathrm{H}(10 B)$ & -7318 & -1994 & 1609 & 30 \\
$\mathrm{H}(10 \mathrm{C})$ & -9121 & -2901 & 1428 & 30 \\
$\mathrm{H}(12)$ & -9455 & -4187 & 691 & 28 \\
$\mathrm{H}(13)$ & -10513 & -6125 & 177 & 34 \\
$\mathrm{H}(14)$ & -9153 & -8530 & 196 & 36 \\
$\mathrm{H}(15)$ & -6765 & -9032 & 757 & 34 \\
$\mathrm{H}(16)$ & -5717 & -7101 & 1283 & 28 \\
$\mathrm{H}(2)$ & -3979 & -4773 & 1095 & 20 \\
$\mathrm{H}(22)$ & -3870 & -1668 & 1944 & 23 \\
$\mathrm{H}(23)$ & -2783 & 791 & 1795 & 25 \\
$\mathrm{H}(24)$ & -1949 & 1524 & 999 & 27 \\
$\mathrm{H}(25)$ & -2267 & -209 & 340 & 26 \\
& & & & \\
\hline
\end{tabular}




\section{References}

(1) (a) Hori, T.; Singer, S. P.; Sharpless, K. B. J. Org. Chem. 1978, 43, 1456-1459. (b) Kresze, G.; Wucherpfennig, W. Angew. Chem., Int. Ed. Engl. 1967, 6, 149-167.

(2) Ruble, J. C.; Fu, G. C. J. Am. Chem. Soc. 1998, 120, 11532-11533.

(3) Lund, E. A.; Kennedy, I. A.; Fallis, A. G. Can. J. Chem. 1996, 74, 2401-2412. Baigre, L. M.; Seiklay, H. R.; Tidwell, T. T. J. Am. Chem. Soc. 1985, 107, 5391-5396. 